\author{
Aus dem Fachbereich Medizin \\ der Johann Wolfgang Goethe-Universität \\ Frankfurt am Main
}

betreut am

Zentrum für Chirurgie

Klinik für Unfall-, Hand- und Wiederherstellungschirurgie

Direktor: Prof. Dr. Ingo Marzi

\title{
Einfluss eines Erste-Hilfe-Kurses für Medizinstudierende auf notfallmedizinische Basiskompetenzen
}

\author{
Dissertation \\ zur Erlangung des Doktorgrades der Medizin \\ des Fachbereichs Medizin \\ der Johann Wolfgang Goethe-Universität \\ Frankfurt am Main \\ vorgelegt von \\ Julia Lorenz \\ aus Nürtingen \\ Frankfurt am Main, 2021
}


Dekan: Prof. Dr. Stefan Zeuzem

Referentin: Prof. Dr. Miriam Rüsseler

Korreferent: Prof. Dr. Udo Rolle

Tag der mündlichen Prüfung: 07.09.2021 
Für meine Eltern \& meine Schwester 


\section{Inhaltsverzeichnis}

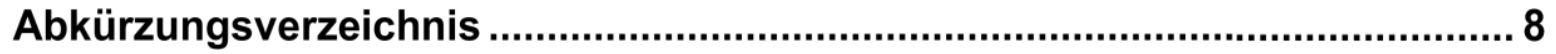

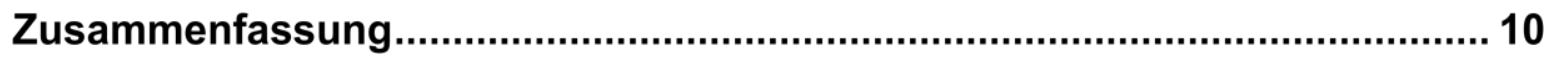

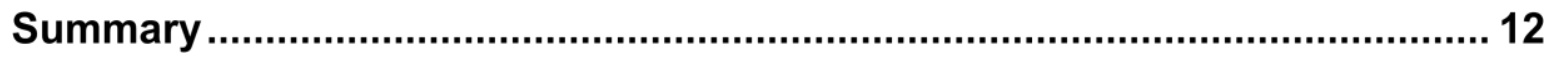

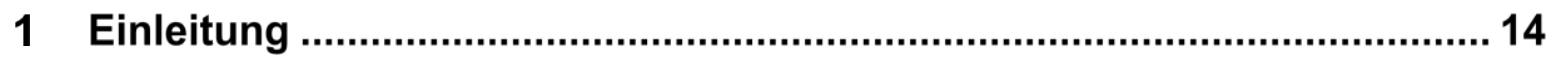

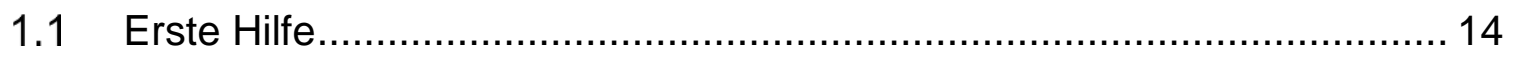

1.1.1 Erste Hilfe in Deutschland .................................................... 14

1.1.2 Rechtliche Grundlagen .............................................................. 14

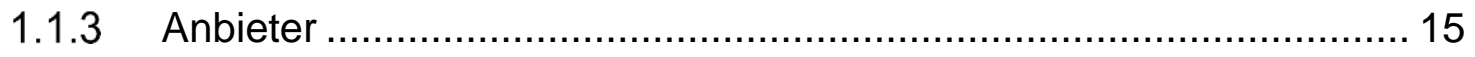

1.1.4 Inhalte der Ersten-Hilfe-Kurse …............................................ 16

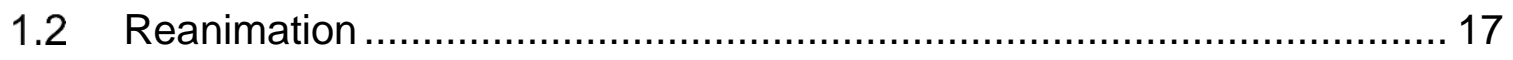

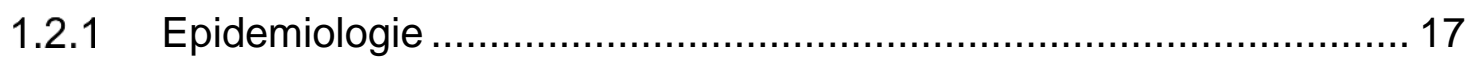

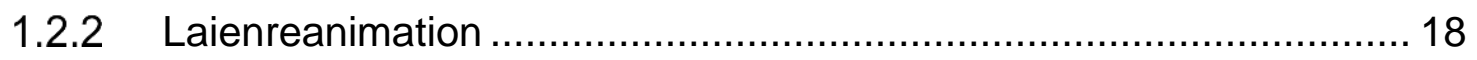

1.2.3 Einsetzen eines AED im Rahmen der Laienreanimation ................... 21

1.3 Notfallmedizinische Ausbildung von Schüler:innen ................................ 22

1.4 Notfallmedizinische Ausbildung von Medizinstudierenden ..................... 23

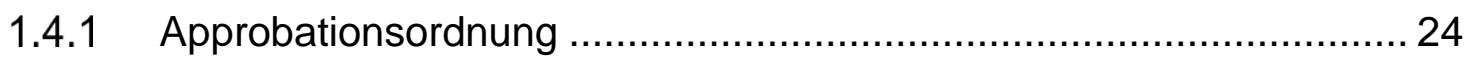

1.4.2 Erste-Hilfe-Schein zur Vorlage für den 1. Abschnitt der Ärztlichen

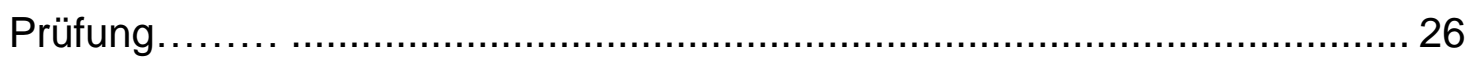

1.4.3 Wissensstand und praktische Kompetenzen der Medizinstudierenden .30

1.5 Standardisierte Aus- und Weiterbildung in der Notfallmedizin.................. 31

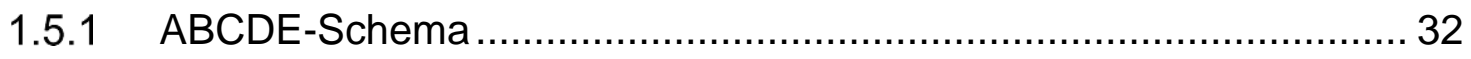

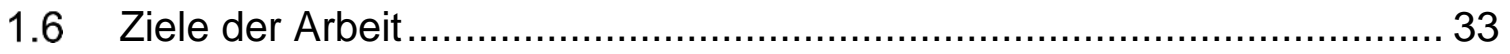




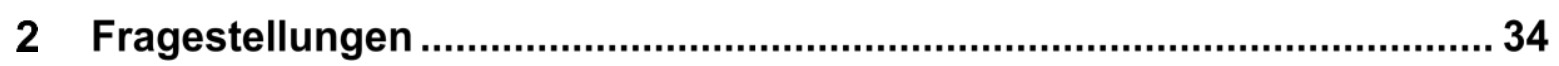

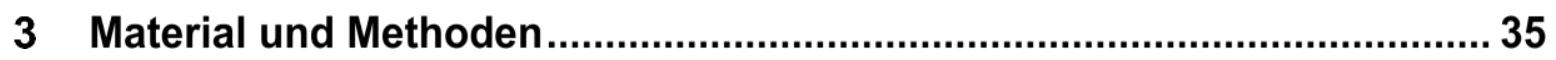

3.1 Konzeption des Erste-Hilfe-Kurses ................................................... 35

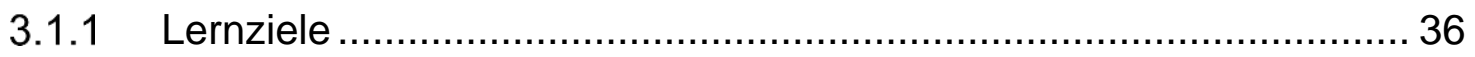

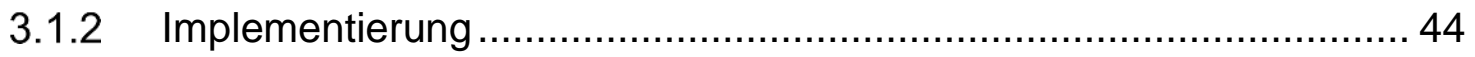

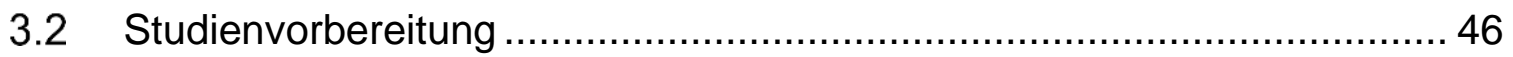

3.2.1 Selbsteinschätzungsbogen ....................................................... 47

3.2.2 Multiple-Choice-Fragen zur Wissensüberprüfung ........................... 48

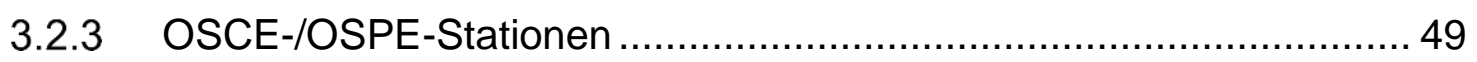

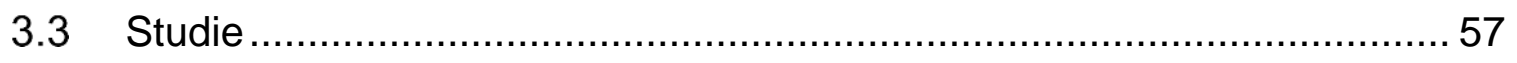

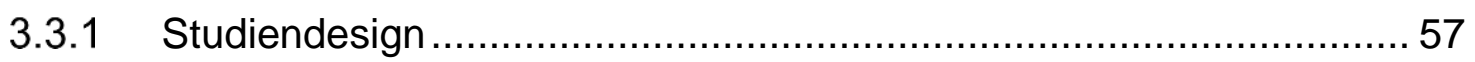

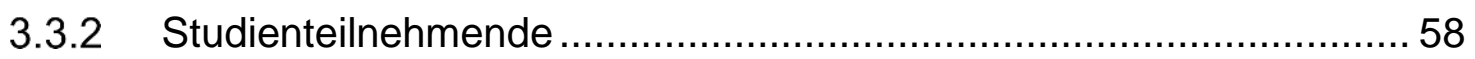

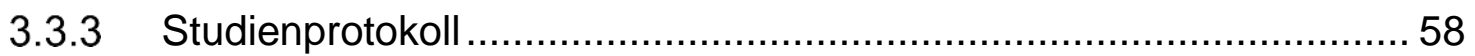

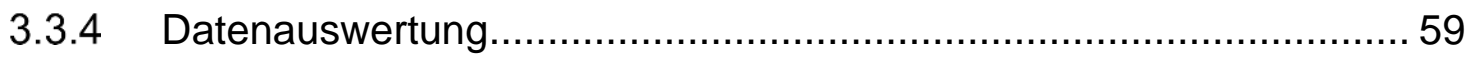

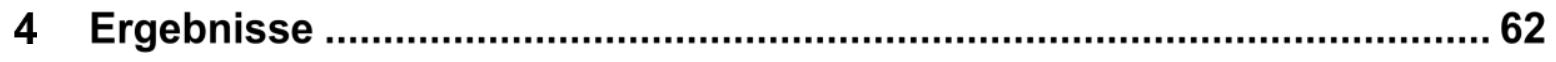

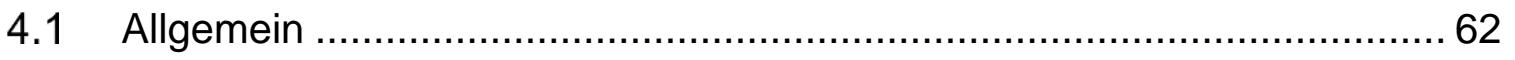

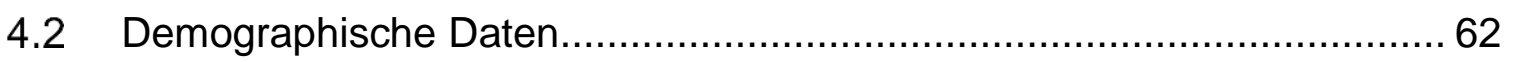

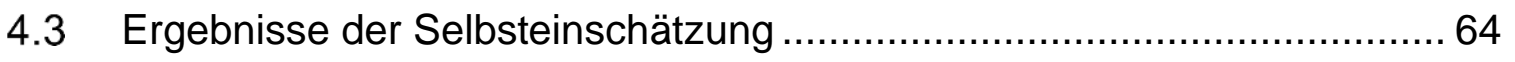

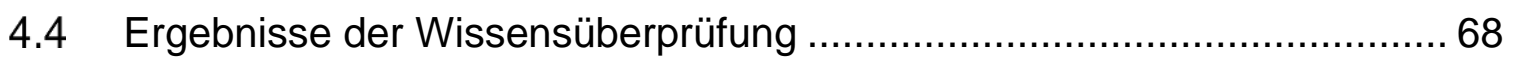

4.5 Ergebnisse der Kompetenzerfassung .............................................. 75

4.5.1 OSCE/OSPE 1 - Ein-Helfer-Reanimation .................................... 76

4.5.2 OSCE/OSPE 2 - Stabile Seitenlage …....................................... 77

4.5.3 OSCE/OSPE 3 - Zwei-Helfer-Reanimation, Einsetzen des AED und Anleitung eines Passanten …..................................................................... 79

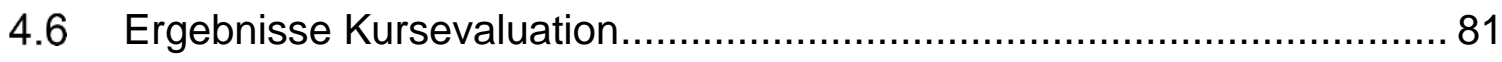




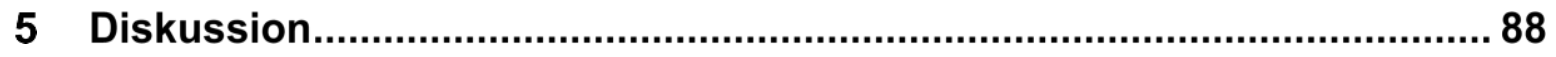

5.1 Zusammenfassung der Ergebnisse vor dem Erste-Hilfe-Kurs................. 88

5.2 Zusammenfassung der Ergebnisse nach dem Erste-Hilfe-Kurs .............. 88

5.3 Vergleich mit bisheriger Literatur................................................... 89

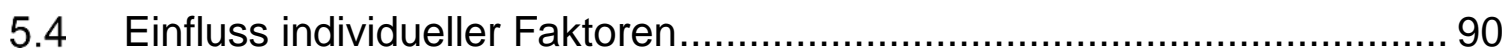

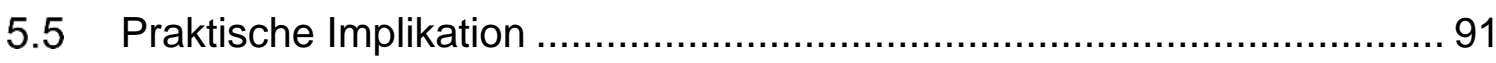

5.5.1 Evaluation des Kurskonzeptes....................................................... 91

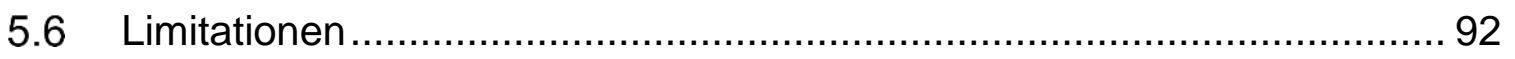

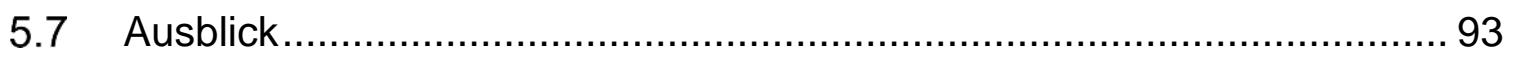

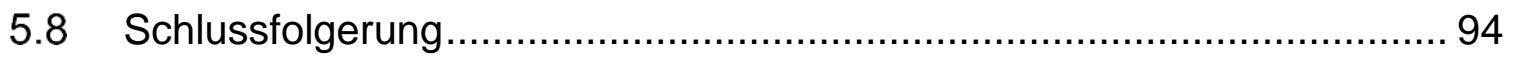

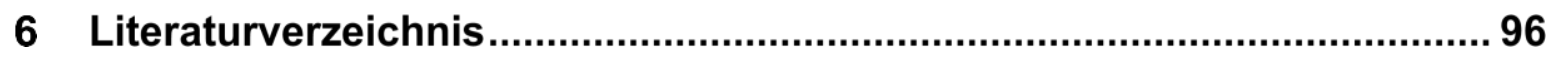

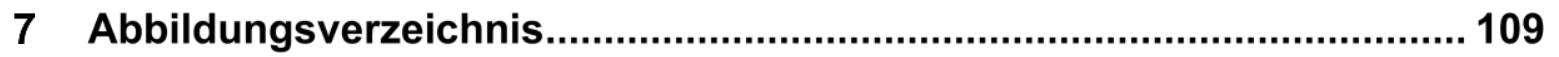

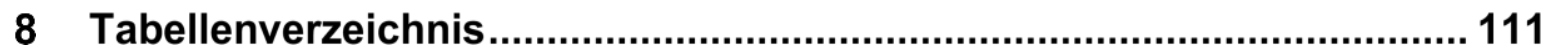

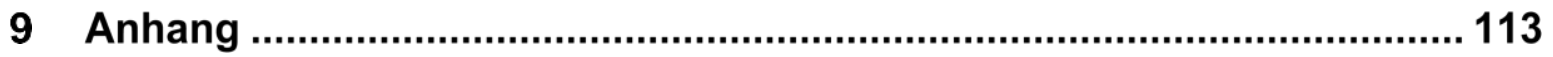

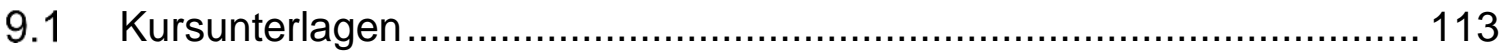

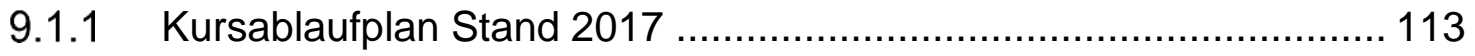

9.1.2 Ausbildungs-Nachweis Instruktoren Stand 2017 ......................... 114

9.1.3 Teilnahmebescheinigung Stand 2017 ....................................... 115

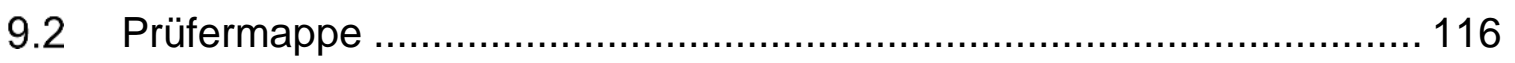

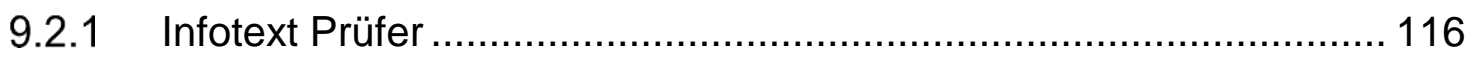

9.2.2 OSCE-Checklisten ................................................................ 117

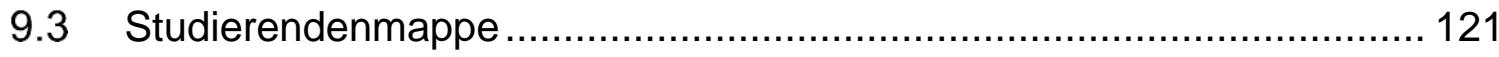

9.3.1 Subjektive Einschätzung vor und nach dem OSCE ....................... 121

9.3.2 MC-Fragen vor und nach dem OSCE ......................................... 122 
9.3.3 Informationstext der 3 OSCE-Stationen...................................... 124

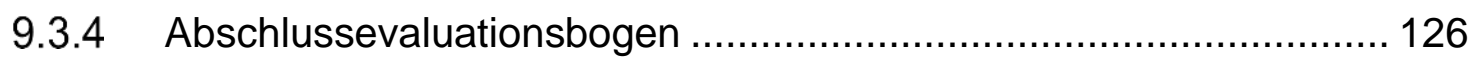

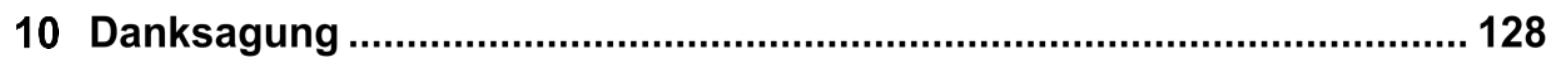

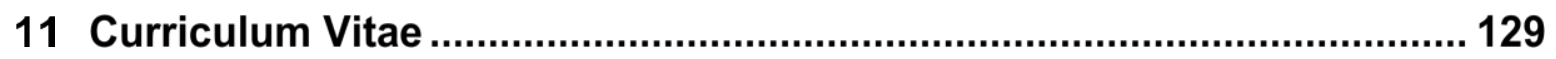

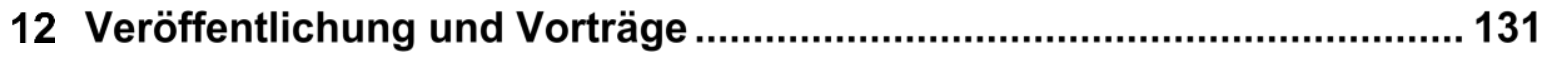

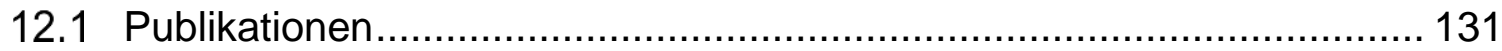

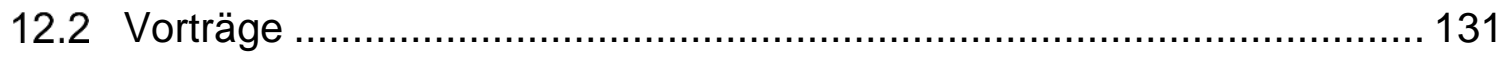

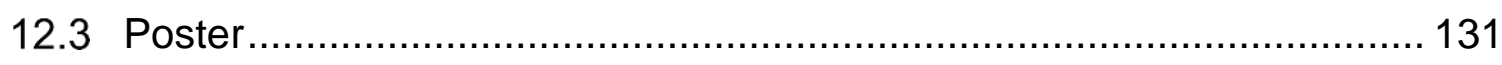

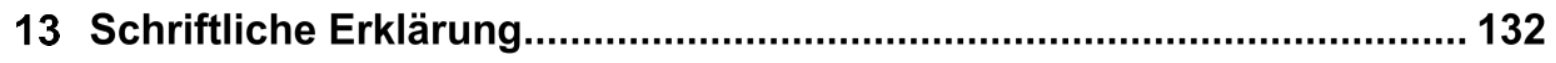




\section{Abkürzungsverzeichnis}

\begin{tabular}{ll} 
ÄAppO & Ärztliche Approbationsordnung \\
ABCDE & Airway, Breathing, Circulation, Disability, Exposure/Environment \\
A(C)LS & Advance (Cardiac) Life Support \\
AED & Automatisierter externer Defibrillator \\
AHA & American Heart Association \\
BLS & Basic Life Support \\
CPR & cardiopulmonary resuscitation \\
DGUV & Deutsche Gesetzliche Unfallversicherung \\
ERC & European Resuscitation Council \\
FIneST & Frankfurter Interdisziplinäres Simulationstraining \\
GRC & German Resuscitation Council \\
HLW & Herz-Lungen-Wiederbelebung \\
ILCOR & International Liaison Committee on Resuscitation \\
MC & Multiple Choice \\
NAEMT & National Association of Emergency Medical Technicians \\
NKLM & Nationaler Kompetenzbasierter Lernzielkatalog \\
OSCE & Objective Structured Clinical Examination \\
OSPE & Objective Structured Practical Examination \\
QB & Querschnittsbereich \\
PAL & Peer Assisted Learning \\
SPSS & Statistical Package of the Social Sciences \\
\hline
\end{tabular}


SWS

Semesterwochenstunden

UE

Unterrichtseinheit 


\section{Zusammenfassung}

\section{Fragestellung}

Medizinstudierende sind bereits in der Vorklinik häufig mit Situationen konfrontiert, in denen medizinische Notfälle passieren, die unter anderem wegen der Lage des Universitätscampus und den damit verbundenen Kontakt zu Patientinnen und Patienten oder in den Pflegepraktika. Tritt in diesen Situationen ein medizinischer Notfall ein, müssen sie Erste Hilfe leisten können. Das Ziel der vorliegenden Arbeit ist daher die Erfassung notfallmedizinischer Basiskompetenzen von Medizinstudierenden der Vorklinik und die Frage, ob diese ausreichend sind, um den Anforderungen ihres Umfeldes gerecht zu werden. Des Weiteren wurde ein Erste-Hilfe-Kurs speziell für Vorklinikstudierende basierend auf dem ABCDE-Schema konzipiert, implementiert und evaluiert, ob die erfassten Kompetenzdefizite behoben werden konnten.

\section{Methodik}

In einem Pre-Post-Design wurde die theoretische Kompetenz anhand eines 10 Items Multiple-Choice-Tests und die praktischen Kompetenzen anhand von 3 OSCE-/OSPE-Stationen (je $5 \mathrm{~min}$ ) erfasst. Sowohl der MC-Test als auch die OSCE/OSPE-Stationen wurden in Probeläufen getestet und überarbeitet. Soziodemographische Daten wurden mittels Fragebogen erhoben. Der entwickelte Kurs umfasst 9 UE (davon 6 UE praktische Übungen) in denen die zu erlernenden Erste-Hilfe-Maßnahmen strukturiert und in logischer Reihenfolge anhand des etablierten ABCDESchemas vermittelt werden. Jeweils 8 Teilnehmende werden von zwei geschulten Peer-Dozierenden unterrichtet. Im Anschluss an das Training erfolgte eine erneute Erhebung der theoretischen und praktischen Kompetenzen. Die Eingabe der Rohdaten erfolgte in Microsoft Excel (Microsoft Inc., Redmond, WA, USA). Die weitere Auswertung erfolgte mittels SPSS (SPSS Inc., Chicago, IL, USA).

\section{Ergebnisse}

160 Studierende aus den ersten beiden Studienjahren konnten in die Studie eingeschlossen werden. Im Basis-Assessment demonstrierten nur 25\% der Studierenden 
zwei Minuten lang eine suffiziente Kompression mit richtiger Frequenz und Drucktiefe, nur 29\% überstreckten am Ende der Seitenlage den Kopf des Patienten und öffneten den Mund. Im Anschluss an das Training erzielten die Studierenden an allen OSCE/OSPE-Stationen signifikant bessere Ergebnisse: Bei der stabilen Seitenlage steigerten sie sich durchschnittlich um 5,3 Punkte (max. 14), bei der Reanimation um 6,8 Punkte (max. 18). Während sich vor dem Kurs 33\% der Studierenden in der Lage fühlten, Wiederbelebungsmaßnahmen durchzuführen, waren dies nach dem Kurs $98 \%$. Die Kursevaluation war durchweg positiv.

\section{Schlussfolgerung}

Die Erfassung notfallmedizinischer Basiskompetenzen von Medizinstudierenden der Vorklinik zeigte, dass diese nicht ausreichend geschult sind, um Notfallsituationen adäquat zu bewältigen. Vor diesem Hintergrund muss eine dem Anforderungsprofil entsprechende Ausbildung in Erster Hilfe bereits in der Vorklinik an der Universität gewährleistet werden. Die vorliegenden Ergebnisse zeigen, dass mithilfe eines speziell auf Medizinstudierende ausgerichteten Erste-Hilfe-Kurs eine deutliche Steigerung notfallmedizinischer Basiskompetenzen erfolgen kann. 


\title{
Summary
}

\begin{abstract}
Aim
Even in the preclinical stage, medical students are often confronted with situations in which medical emergencies occur, e.g. through the location of the university campus and the associated contact with patients or during nursing internships. If a medical emergency occurs in these situations, they must be able to provide first aid. Therefore, the aim of this study is to assess the basic emergency medical skills of preclinical medical students and whether they are sufficient to meet the requirements of their environment. Furthermore, a first aid course especially for preclinical students based on the ABCDE scheme was designed, implemented and evaluated whether the recorded competence deficits could be remedied.
\end{abstract}

\section{Methods}

In a pre-post design, theoretical competency was assessed using a 10-item multiple choice test, and practical competency was assessed using 3 OSCE/OSPE-stations (5 min each). Both, the MC-test and the OSCE-/OSPE-stations were tested and revised in trial runs. Sociodemographic data were collected using questionnaires. The developed course comprises 9 lessons (including 6 lessons of practical exercises) in which the first aid measures to be learned are taught in a structured and logical sequence using the established $\mathrm{ABCDE}$-scheme. In each case 8 participants are taught by two trained peer instructores. Following the training, a new survey of theoretical and practical competencies was conducted. The raw data was entered into Microsoft Excel (Microsoft Inc., Redmond, WA, USA). Further analysis was conducted using SPSS (SPSS Inc., Chicago, IL, USA).

\section{Results}

160 students from the first two years of study were included in the study. In the baseline assessment, only $25 \%$ of the students demonstrated sufficient compression with correct frequency and depth of pressure for two minutes, and $29 \%$ hyperextended the patient's head and opened the mouth at the end of the lateral position. 
Following training, students performed significantly better at all OSCE-/OSPE-stations: They increased by an average of 5.3 points (max. 14) in the stable lateral position and by 6.8 points (max. 18) in resuscitation. While $33 \%$ of the students felt able to perform resuscitation before the course, $98 \%$ did so after the course. Course evaluation was consistently positive.

\section{Conclusion}

The assessment of basic emergency skills of preclinical medical students showed that they are not sufficiently trained to adequately handle emergency situations. Against this background, training in first aid in line with the requirements profile must be ensured already in the preclinical phase at the university. The present results show that a first aid course specifically designed for medical students can significantly increase basic emergency medical skills. 


\section{Einleitung}

\subsection{Erste Hilfe}

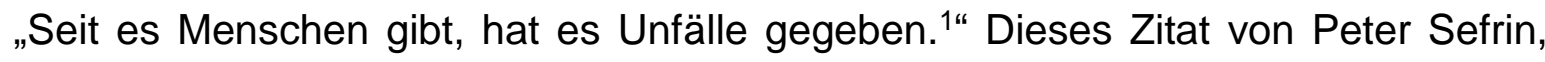
einem der bekanntesten deutschen Notfall- und Katastrophenmediziner, legt nahe, dass die Erste Hilfe seit jeher fest zur praktischen Medizin gehört. Zunächst rein ärztlich ausgeführt, wurde mit der strukturierten Versorgung durch den Rettungsdienst auch die Breitenausbildung bedeutender, ${ }^{1}$ da sich in über $90 \%$ der Notfälle Ersthelfer:innen vor Ort befinden, die direkt handeln können.. ${ }^{2,3}$ Insbesondere mit dem Militär entwickelten sich verschiedene Erste-Hilfe- und Wiederbelebungstechniken, die nicht nur dort, sondern auch in neu gegründeten Verbänden für Erste Hilfe umgesetzt und an die Bevölkerung weitergegeben wurden.

\subsubsection{Erste Hilfe in Deutschland}

Heutzutage ist in Deutschland ein Erste-Hilfe-Kurs vor allem zum Erwerb der verschieden Führerscheinklassen und im Rahmen der Notfallvorsorge in Betrieben Pflicht. Zusätzlich ist er seit der Approbationsordnung für Ärzt:innen vom 1. Oktober 1972 Voraussetzung für die Zulassung zum 1. Abschnitt der Ärztlichen Prüfung („Physikum“) für Medizinstudierende. ${ }^{4}$

Der Umfang des Kurses beträgt seit dem 01. April 2015 neun Unterrichtseinheiten (UE). ${ }^{5}$ Dabei besteht keine gesetzliche Wiederholungspflicht, einzig die Berufsgenossenschaften setzen eine Wiederholung innerhalb von zwei Jahren voraus. In den Lehrgängen erfolgt keine Prüfung des erworbenen Wissens und der praktischen Fähigkeiten, die Teilnehmenden erhalten lediglich eine Bescheinigung über ihre Anwesenheit. $^{6}$

\subsubsection{Rechtliche Grundlagen}

In Deutschland sind alle Personen gemäß § 323c Strafgesetzbuch dazu verpflichtet, in Notsituationen Erste Hilfe zu leisten. ${ }^{7}$ Eine unterlassene Hilfeleistung kann mit 
einer Geld- oder Freiheitsstrafe bestraft werden. Ärzt:innen und ausgebildetes medizinisches Fachpersonal sind verpflichtet, ihrer Qualifikation nach zu handeln und die Regeln des Basic Life Supports (BLS) einzuhalten. Medizinstudierende zählen dabei nicht zu dieser Gruppe.

Ersthelfer:innen haben bei Fehlern bei Erste-Hilfe-Leistungen mit keinen rechtlichen Konsequenzen zu rechnen. Allerdings darf nicht fahrlässig und vorsätzlich fehlerhaft gehandelt werden und die Maßnahmen und Fähigkeiten müssen den aktuellen Leitlinien folgen. ${ }^{8}$

\subsubsection{Anbieter}

Gängige Anbieter in Deutschland sind die nachfolgend genannten Hilfsorganisationen, die Deutsche Gesetzliche Unfallversicherung (DGUV) und zugelassene private Bildungsanbieter.

Die Hilfsorganisationen haben sich 1988 in der „Bundesarbeitsgemeinschaft Erste Hilfe" zusammengeschlossen. Dazu zählen der Arbeiter-Samariter-Bund e.V., das Deutsche Rote Kreuz e.V., die Johanniter-Unfall-Hilfe e.V. und der Malteser Hilfsdienst e.V. Seit 1994 ist die Deutsche Lebens-Rettungs-Gesellschaft e.V. Teil der Arbeitsgemeinschaft. ${ }^{9}$ Ziele der Arbeitsgemeinschaft sind unter anderem eine Beratung und Abstimmung in den Aufgabenbereichen der Ersten Hilfe, inklusive der Ausarbeitung von Stellungnahmen und Empfehlungen zur Ersten Hilfe. ${ }^{10}$

Zusätzlich haben sich im „Verband der privaten Erste Hilfe Schulen“ 12 Erste-HilfeSchulen und Lehrkräfte der notfallmedizinischen Ausbildung zusammengeschlossen. Der Verband soll beispielsweise Ansprechpartner für Stellen des Bundes, der Länder, der Berufsgenossenschaften, anderer Organisationen und Verbände sein. Ziel ist insbesondere, eine freiwillige und einheitliche Qualitätssicherung zu schaffen. Die deutschen Verbände unterstellen sich fast alle den gängigen Leitlinien des German Resuscitation Council (GRC) bzw. nutzen diese als Grundlage für ihre Empfehlungen und Erste-Hilfe-Kurse. 


\subsubsection{Inhalte der Ersten-Hilfe-Kurse}

Die Erste-Hilfe-Kurse folgen keinem deutschlandweit einheitlichen, strukturierten Schema oder festen Vorgaben. Es gibt allerdings durch die oben genannten Verbände Empfehlungen für die Kurse, u.a. das Kapitel 9 „Erste Hilfe“ im Rahmen der 2015 veröffentlichten Leitlinien zur Reanimation des European Resuscitation Council (ERC). ${ }^{11}$

Die Inhalte sind bei einem Großteil der Kurse die gleichen, vorrangig wird sich an der Rettungskette mit ihren vier Gliedern orientiert (vgl. Abb. 1).
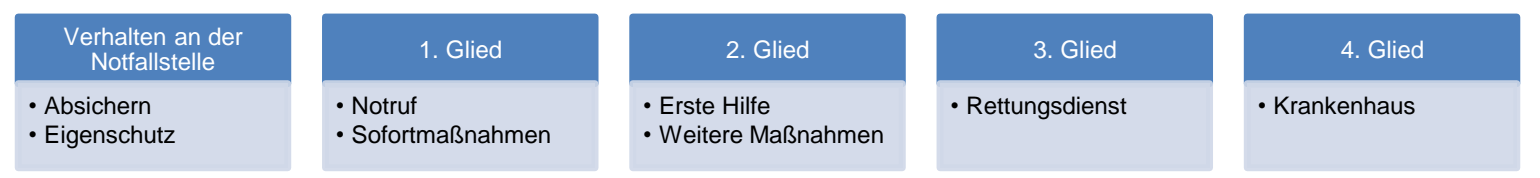

Abbildung 1: Beispielhafte Darstellung der Rettungskette. Eigene Darstellung.

Bereits in den 1960er Jahren wurde durch Ahnefeld et al. ${ }^{12}$ in Deutschland der Begriff der Rettungskette geprägt, mithilfe derer die chronologische Abfolge eines Rettungseinsatzes veranschaulicht wird und an der sich in Notfallsituationen orientiert werden kann. Aus der Rettungskette ist ersichtlich, dass die Basis einer optimalen Notfallversorgung bereits durch Laienretter:innen gegeben sein muss. Eine zeitige Alarmierung und die Durchführung der erforderlichen Maßnahmen sind lebensnotwendig: „Wie bei einer Kette ist für den Patienten das Überleben mit gutem Outcome vom schwächsten Kettenglied abhängig. ${ }^{7 "}$

Aufgrund dessen wird in den meisten Erste-Hilfe-Kursen der Fokus auf die praktischen Maßnahmen gelegt. Elementar sind hierbei Wiederbelebungsmaßnahmen, Wund- und Frakturversorgung, Blutstillung, Erkennen eines Schlaganfalls, Vorgehen bei einem Krampfanfall, thermische Schädigungen behandeln, Schock als lebensbedrohlicher Zustand und Herz-Kreislauf-Störungen erkennen und erstversorgen. ${ }^{7}$ 


\subsection{Reanimation}

\subsubsection{Epidemiologie}

Der Herz-Kreislauf-Stillstand stellt nach wie vor die häufigste Todesursache in Deutschland dar. ${ }^{13}$

Todesursachen nach Krankheitsarten 2018

in \%

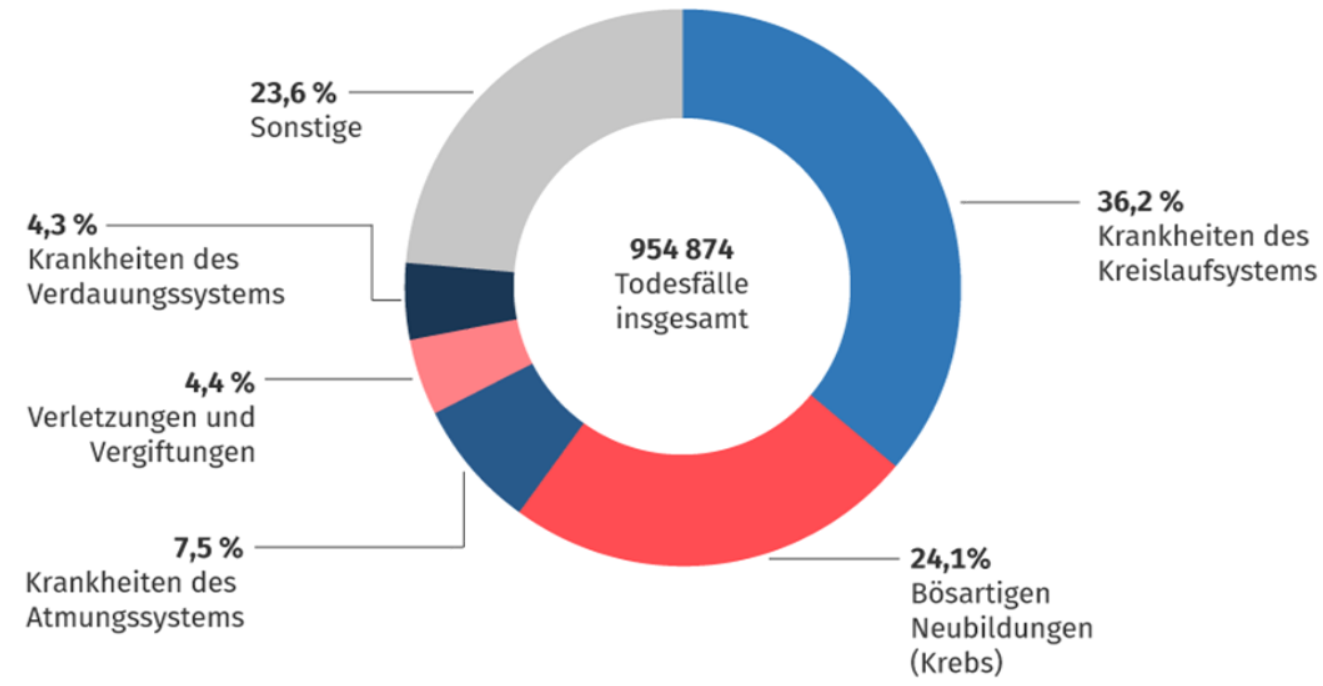

(c) Statistisches Bundesamt (Destatis), 2020

Abbildung 2: Todesursachen nach Krankheitsarten 2018, Statistisches Bundesamt. ${ }^{14}$

2018 lag laut der Statistik des Deutschen Reanimationsregisters der Mittelwert aller außerklinischen Herz-Kreislauf-Stillstände in Deutschland bei 121 Patienten pro 100.000 Einwohner im Jahr. ${ }^{13}$ Nur 13\% der außerklinisch reanimierten Patienten konnten lebend aus dem Krankenhaus entlassen werden. In 44\% der Fälle fand eine Reanimation durch Laien vor Eintreffen des Rettungsdienstes statt, um die im Mittel ca. 7 Minuten dauernde Anfahrt bis zum Eintreffen des ersten Rettungsfahrzeuges zu überbücken. ${ }^{13}$ Auch wenn Christ et al. ${ }^{15}$ zeigen konnten, dass Laien die meisten 
Herz-Kreislauf-Stillstände in Deutschland beobachteten, liegt Deutschland im internationalen Vergleich bei der Anzahl der Laienreanimation auf den hinteren Plätzen. ${ }^{16}$

\subsubsection{Laienreanimation}

Das International Liasion Committee on Resuscitation (ILCOR), u.a. bestehend aus der American Heart Association (AHA) und dem European Resuscitation Council $(E R C)$, ist weltweiter Dachverband und gibt regelmäßig Handlungsempfehlungen zur Reanimation heraus. ${ }^{17}$ Die aktuelle Empfehlung für Ersthelfer:innen lautet, dass sie bei reanimationspflichtigen Patient:innen Thoraxkompressionen sowie Beatmungen durchführen sollen, sofern sie dahingehend ausreichend trainiert sind. ${ }^{17}$ 


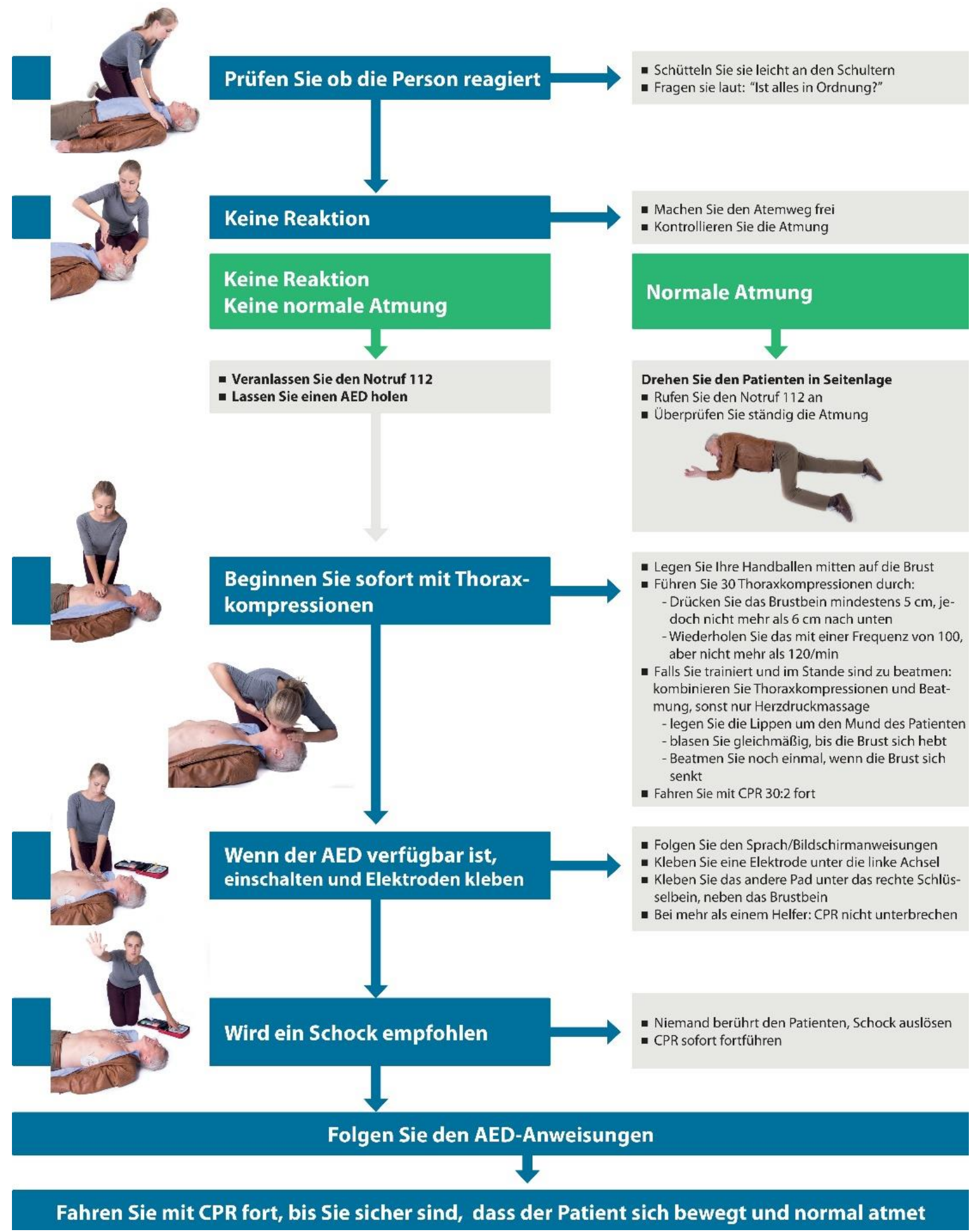

Abbildung 3: Laienreanimation nach Empfehlung der GRC. ${ }^{18}$ 
In mehreren Studien konnte durch diese Empfehlung der Herz-Thorax-Kompression und Beatmung durch Laien ein positiver Effekt auf das Überleben der Patient:innen nachgewiesen werden. ${ }^{19-24} \mathrm{Bspw}$. zeigt die Studie von Hasselqvist-Ax et al. ${ }^{19}$, dass die 30-Tage-Überlebensrate von Patient:innen, die vor Eintreffen des Rettungsteams reanimiert wurden, zweifach höher lag als bei Patient:innen, die erst bei Eintreffen des ersten Rettungsmittels behandelt wurden.

Die neusten veröffentlichten Zahlen des Deutschen Reanimationsregisters aus dem Jahr 2018 zeigen jedoch, dass 86,8\% der Patient:innen einen Herz-Kreislauf-Stillstand in Deutschland nicht überlebten. Im europäischen Vergleich zu bspw. Dänemark, wo nach der EuReCa TWO-Studie statistisch gesehen die meisten Patient:innen nach einer Out-of-hospital Reanimation überleben, schneidet Deutschland im Vergleich schlechter ab. ${ }^{25}$ Die Gründe hierfür sind zahlreich, z.B. begannen weniger als die Hälfte der Laien eine Herzdruckmassage. ${ }^{13}$ Auch mangelndes Training in diesem Bereich scheint zur unzureichenden Durchführung beizutragen. ${ }^{26,27}$ In einer von Breckwoldt et al. ${ }^{28}$ durchgeführten Studie konnte gezeigt werden, dass in ErsteHilfe-Kursen in Deutschland oftmals die praktischen Übungszeiten zu kurz waren und gravierende standardisierte Fehler der Teilnehmenden nicht korrigiert wurden. Erfreulicherweise steigt die Anzahl der Laienreanimationen in Deutschland weiterhin an. ${ }^{23,29}$ Verantwortlich dafür sind bspw. die telefonischen Anleitung zur Reanimation am Telefon durch Leitstellen, bundesweite Aktionen wie die „Woche der Wiederbelebung“ sowie „weitreichende konzeptionelle Veränderungen der „Erste-HilfeKurse"29", die seit Anfang 2015 in Umsetzung sind und nun v.a. die praktischen Kursinhalte betonen". ${ }^{29}$

Insgesamt betrachtet scheint die Laienreanimation eine unsichere Variable in der Versorgung reanimationspflichtiger Patient:innen zu sein: In verschiedenen Studien zeigte sich eine mangelnde CPR-Qualität durch Laien, weshalb Expert:innen eine höhere Frequenz und Regelmäßigkeit von CPR-Trainings empfehlen, um nicht nur die Qualität, sondern auch das Bewusstsein in der Gesellschaft zu verbessern. $2,15,26,30-37$ 
Eine Studie von Winkler et al. zeigte, dass der Anteil von praktischen Übungen der Herz-Lungen-Wiederbelebung (HLW) in Erste-Hilfe-Kursen gering ist, „während andere, nicht primär lebensrettende Maßnahmen ausführlich behandelt werden." Expert:innen fordern daher „die inhaltliche Gestaltung von Erste-Hilfe-Kursen zu überdenken6“ und bspw. eine „verpflichtende prüfungsartige Demonstration einer Wiederbelebung ${ }^{6 "}$ einzuführen, um „den Wissenszuwachs und das praktische Können [zu] verbessern6“ und bei „unmotivierten Teilnehmern ein reines ,Aussitzen“ des Kurses $^{6 “}$ zu verhindern. Zudem wird empfohlen, dass „die Kompetenz frühzeitig im Verlauf des lebenslangen Lernens als Kulturfertigkeit verankert wird, wie etwa bei der Ausbildung von Schülern in Wiederbelebungsmaßnahmen ${ }^{23 "}$ und die Kurse attraktiver gestaltet werden sollen, bspw. durch inhaltlich und zeitlich auf die Zielgruppe angepasste Kursformate. ${ }^{16}$ „Das alleinige Angebot der vier bzw. acht Doppelstunden dauernden Kurse in lebensrettenden Sofortmaßnahmen und Erster Hilfe scheinen [...] nicht mehr zeitgemäß zu sein. ${ }^{16 "}$

\subsubsection{Einsetzen eines AED im Rahmen der Laienreanimation}

Verschiedene Arbeiten zeigen, dass das Outcome der Patient:innen durch das frühe Einsetzen einer Defibrillation positiv beeinflusst wird. ${ }^{38-40}$ Die modernen automatisierten externen Defibrillatoren (AED) sind einfach zu bedienen, erkennen sicher die Herzrhythmen und zeigen bei Nutzung eine effektive Verbesserung des Outcomes bei Herzstillstand. ${ }^{41}$ Einfach konzipierte Geräte ermöglichen mittels Sprachkommandos eine Anwendungssicherheit, sodass auch Ersthelfer:innen den AED zu lebensrettenden Maßnahmen nutzen können. ${ }^{42}$ Als Medizinprodukt darf das Gerät im rechtfertigenden Notstand ( $§ 34$ StGB) auch von nicht eingewiesenem Personal an Patient:innen angewendet werden. Die Anwendung zieht somit keine rechtlichen Konsequenzen nach sich. ${ }^{43}$

Mehrere Studien zeigen, dass der frühzeitige Einsatz eines AED durch Ersthelfer:innen möglich ist. ${ }^{37,44-4645}$ Aus diesem Grund sollen laut den aktuellen Empfehlungen auch Laien möglichst frühzeitig einen AED einsetzen. ${ }^{47}$ Obwohl gezeigt werden konnte, dass selbst Kleinkinder die Anweisungen eines AED befolgen können, wird 
ein Training zur Anwendung empfohlen, um bspw. die Zeit bis zur ersten Defibrillation in der Notfallsituation zu reduzieren. ${ }^{40,48}$ Lackner et al. ${ }^{49}$ konnten zeigen, dass die sichere Anwendung des AED schon nach einer kurzen Schulung möglich war. Die Bundesarbeitsgemeinschaft Erste Hilfe reagierte 2003 mit der Aus- und Fortbildung von Ersthelfer:innen in der Frühdefibrillation im Rahmen eines AED-Grundlehrgangs, -Aufbaulehrgangs, -Ergänzungslehrgangs oder einer AED-Fortbildung. ${ }^{50}$ 2011 folgte die Entscheidung, in jedem Erste-Hilfe-Kurs einen AED vorzustellen. Ihrer Meinung nach sollen „besonders Bevölkerungsgruppen, die aufgrund ihres Berufs oder ihres Alters für die Rolle eines potentiellen Ersthelfers prädestiniert ${ }^{49 \text { " }}$ seien, in die Lage versetzt werden, AEDs zielführend und sicher zu bedienen. ${ }^{49}$

Doch nur wenige Laien trauen sich eine Nutzung des AEDs zu, ${ }^{36}$ da die Angst überwiegt, jemanden mit einem AED zu verletzen. ${ }^{51}$ Dies müsste durch intensive Schulung im Rahmen regelmäßiger Trainings verbessert werden. ${ }^{51}$ So würden die Hemmungen, im Notfall einen AED einzusetzen sinken und auch Anwendungsfehler, die sich durch die erstmalige Verwendung des AED ergeben, würden reduziert werden, wie bspw. die Verlängerung der No-flow-time durch fehlende Thoraxkompressionen bei der Reanimation. ${ }^{52}$

\subsection{Notfallmedizinische Ausbildung von Schüler:innen}

Es konnte gezeigt werden, dass Länder, die Wiederbelebung als Schulfach integrieren konnten, mit 50-75\% signifikant höhere Laienreanimationsquoten haben, als andere Länder. ${ }^{53}$ Aus diesem Grund empfehlen Expert:innen, die WHO als auch die deutsche Kultusministerkonferenz, dass Kurse zu lebensrettenden Sofortmaßnahmen bereits in den Schulen durchgeführt werden sollen.2,29,34 Weltweite Aktionen und Projekte in Schulen haben gezeigt, dass die Einführung des Schulfachs „Wiederbelebung" an Schulen möglich ist. ${ }^{53}$

Auch hier sollte das Ziel sein, „durch wiederholtes Training eine Verbesserung der Erste-Hilfe-Leistung zu erreichen“" da sich in vielen Studien ein mangelndes Wissen 
und Können der Schüler:innen gezeigt hat. ${ }^{54-56}$ Gräsner et al. ${ }^{57}$ berichten: „Wiederbelebung ist einfach zu erlernen und muss bereits im Schulalter trainiert werden. Durch die frühe Auseinandersetzung mit dem Thema werden Berührungsängste vermieden und Wiederbelebung wird zu einer Selbstverständlichkeit. Eine Integration in den Lehrplan und das regelmäßige Wiederholen der Schulung fördert diesen Effekt. ${ }^{57}$ "Breckwoldt et al. ${ }^{58}$ fassen zusammen, dass mit einem verpflichtenden Kurs für Schulen alle Bevölkerungsschichten erreicht werden, Verantwortungsbewusstsein frühzeitig verankert werden kann, die Instruktionsqualität höher ist, ein stufenweiser Aufbau von Kompetenzen nötig und eine Verknüpfung mit schulischen Themen möglich ist. Das Wiederbelebungs-Training sollte bereits in frühestmöglichem Alter anfangen und in regelmäßigen Abständen während der Schulzeit wiederholt werden. ${ }^{59}$ Auch "die Integration eines AED in den Wiederbelebungsunterricht an Schulen ${ }^{53 "}$ ist nach Lukas et al. ${ }^{53}$ sinnvoll.

Es gibt verschiedene Studien, die zeigen, dass zusätzlich zu professionellen Instruktor:innen sowohl geschultes Lehrpersonal als auch geschulte Peer-Dozierende für eine adäquate Ausbildung in Erster Hilfe bei Schüler:innen eingesetzt werden können. ${ }^{53,55,60}$ Dabei reichen 45-minütige Trainings bereits aus, um sie für das Ersteintreffen bei einem Herzkreislaufstillstand zu schulen. ${ }^{54}$

\subsection{Notfallmedizinische Ausbildung von Medizinstudierenden}

Erste Hilfe und CPR sind wichtige medizinische Maßnahmen im klinischen Setting. Auch für Medizinstudierende als potenziell Ersteintreffende bei einem klinischen Notfall sind dies wichtige Kompetenzen, die möglichst früh im Studium erlernt werden sollten. ${ }^{61}$ Zum einen scheinen die Erwartungen der Gesellschaft an die Medizinstudierenden hinsichtlich der Beherrschung notfallmedizinischer Maßnahmen hoch zu sein, zum anderen auch der persönliche Anspruch, in Notfallsituationen adäquat helfen und in einem klinischen Setting im Rahmen eines Notfallteams unterstützen zu können. ${ }^{61}$ 
Medizinstudierende sind aufgrund ihres Studiums öfter mit Situationen konfrontiert, in denen Notfälle passieren können. Beispiele hierfür sind die Lage des Campus in direkter Nähe zur Universitätsklinik und den damit verbundenen Kontakt zu Patient:innen. Auch die zahlreich stattfindenden vorklinischen Praktika im Labor stellen einen Risikobereich für Notfälle dar. Spätestens in den für den 1. Abschnitt der Ärztlichen Prüfung vorgeschriebenen Pflegepraktika begegnen die Studierenden das erste Mal Patient:innen, die tendenziell in eine Notfallsituation geraten können. Gerade hier sollten die Studierenden adäquat geschult sein, um handlungsfähig zu sein.

\subsubsection{Approbationsordnung}

Die Notfallmedizin ist ein elementarer Teil der ärztlichen Ausbildung, demnach gilt auch die Ausbildung in Erster Hilfe als zentrale Voraussetzung für die Vorbereitung von Medizinstudierenden auf den ersten Abschnitt der Ärztlichen Prüfung.

Schon zu Beginn der Approbationsordnung ist dies aufgeführt:

„(2) Die ärztliche Ausbildung umfasst

1. ein Studium der Medizin von sechs Jahren an einer Universität oder gleichgestellten Hochschule (Universität), das, vorbehaltlich § 3 Abs. 3 Satz 2, eine zusammenhängende praktische Ausbildung (Praktisches Jahr) von 48 Wochen einschließt;

2. eine Ausbildung in erster Hilfe;

3. einen Krankenpflegedienst von drei Monaten;

4. eine Famulatur von vier Monaten und

5. die Ärztliche Prüfung, die in zwei Abschnitten abzulegen ist. ${ }^{62 “}$

Im Folgenden wird explizit betont: 
„(1) Die Ausbildung in erster Hilfe (§ 1 Abs. 2 Satz 1 Nr. 2) soll durch theoretischen Unterricht und praktische Unterweisungen gründliches Wissen und praktisches Können in erster Hilfe vermitteln.“ ( $\$ 5$, Absatz 1).

(3) Die Teilnahme an einer Ausbildung in erster Hilfe ist bei der Meldung zum Ersten Abschnitt der Ärztlichen Prüfung nachzuweisen. ${ }^{62 “}$

Die Erste-Hilfe-Kurse werden durch die Universitäten nicht vorgegeben und sind nicht fest in das Curriculum der Vorklinik integriert. Im klinischen Studienabschnitt sind Leistungsnachweise im Querschnittsbereich 8 „Notfallmedizin“ zu erbringen. Hier legen die Universitäten selbst mit ihrer Studienordnung die genaue Vermittlung der Querschnittsbereiche fest. Innen steht dafür eine Gesamtstundenzahl für die Fächer und Querschnittsbereiche von mindestens 868 Stunden zur Verfügung. ${ }^{62}$ Jede medizinische Fakultät Deutschlands verfolgt hier unterschiedliche Konzepte im Bereich der notfallmedizinischen Ausbildung. ${ }^{63}$

In einer bundesweiten Umfrage von Beckers et al. ${ }^{63}$ konnte gezeigt werden, dass BLS- und ALS-Kurse in allen medizinischen Fakultäten implementiert sind, aber die Ausbildungszeit zwischen 3 und 13 Unterrichtseinheiten erheblich variiert.

\subsubsection{Querschnittsbereich 8 am Beispiel der Goethe-Universität Frankfurt}

An der Goethe-Universität Frankfurt beinhaltet der Querschnittsbereich 8 aktuell im 5. Semester die Vorlesung „Notfallmedizin I, Erste ärztliche Hilfe“ mit 1,14 Semesterwochenstunden (SWS) und im 7. Semester „Notfallmedizin II“ mit 2,30 SWS. ${ }^{64}$ Beide Vorlesungen schließen mit einer zu bestehenden Abschlussklausur ab. Anschließend erfolgt im 8. Semester das Praktikum „Notfallmedizin“ mit 1,48 SWS. ${ }^{64}$ Fünf Jahre zuvor gab es die Vorlesung im 5. Semester „Notfallmedizin I, Erste ärztliche Hilfe“ mit 1,00 SWS und im 7. Semester „Notfallmedizin II“ mit 2,30 SWS. Zusätzlich fand ein Praktikum „Notfallmedizin I, Erste ärztliche Hilfe“ mit 0,14 SWS im 5. Semester und das „Notfallmedizin III“-Praktikum mit 3,76 SWS im 9. Semester statt. Insgesamt wurde der Querschnittsbereich 8 bis heute um 2,28 SWS gekürzt. ${ }^{65}$ 
Bei einer aktuellen Gesamtanzahl von 166,67 klinischen SWS (Stand 2020) macht der Querschnittsbereich 8 Notfallmedizin ca. 3\% der klinischen Ausbildung aus. Wenn nur die reine praktische Ausbildung in Form des Praktikums betrachtet wird, nimmt die notfallmedizinische Ausbildung ca. 0,9\% des gesamten klinischen Studiums ein.

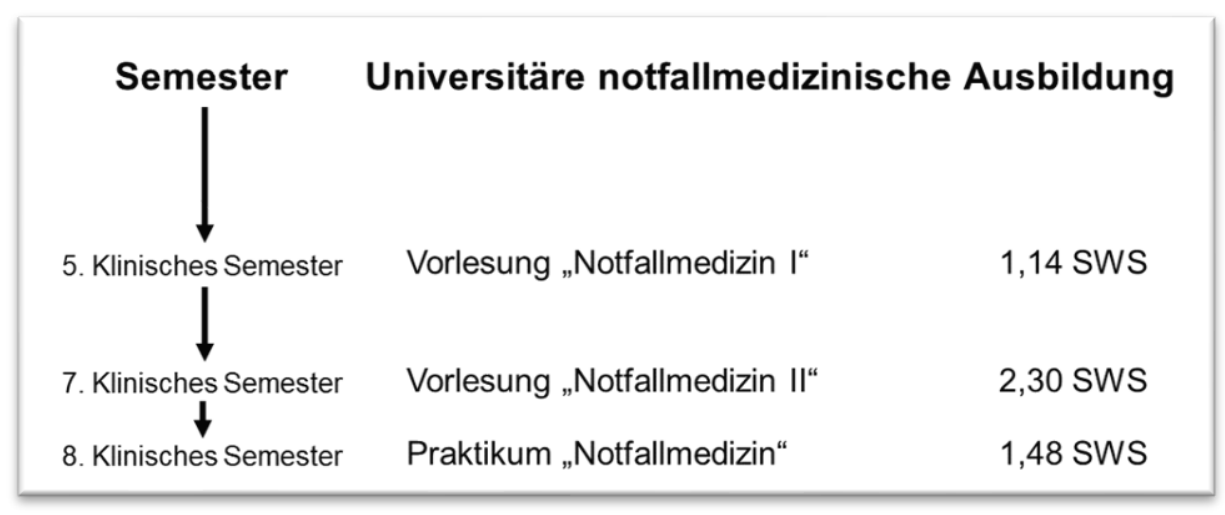

Abbildung 4: Universitäre notfallmedizinische Ausbildung am Beispiel des QB 8 der Goethe-Universität Frankfurt, Stand 2020.

\subsubsection{Erste-Hilfe-Schein zur Vorlage für den 1. Abschnitt der Ärztlichen Prü- fung}

Die Studierenden sind verpflichtet, bei Anmeldung zum 1. Abschnitt der Ärztlichen Prüfung einen Erste-Hilfe-Schein vorzulegen. Die Ausbildung soll über einen Mindestumfang von 9 Unterrichtseinheiten à 45 Minuten gehen. Exkludiert sind Studierende, die eine Berufsausbildung als Krankenpfleger:in, Kinderkrankenpfleger:in, Rettungsassistent:in, Rettungssanitäter:in, Medizinisch-technische Assistent:in, Anästhesietechnische Assistent:in oder Operationstechnische Assistent:in abgeschlossen haben.

Generell gilt, dass ein einmal absolvierter Erste-Hilfe-Kurs nicht verfällt, es sei denn, die Ausbildungsstätte hat dies explizit auf der Teilnahmebescheinigung erwähnt. Die Wahl, welcher Kurs für den vorzulegenden Schein gewählt wird, liegt bei den Studierenden selbst. Aufgrund der zahlreichen Angebote beispielsweise durch den Malteser Hilfsdienst, das Deutsche Rote Kreuz und auch private Anbieter, gibt es keinen 
einheitlichen Qualitätsstandard dieser Kurse. Gerade in sehr kostengünstigen, externen Kursen von privaten Firmen werden viele Teilnehmende untergebracht. Aufgrund der großen Gruppengröße ist es häufig nicht möglich, effektiv praktische Maßnahmen zu üben.

Einige Universitäten haben die beschriebene Problematik bereits erkannt und reagiert: An über $50 \%$ der medizinischen Fakultäten werden Erste-Hilfe-Kurse für Vorklinikstudierende angeboten.

\begin{tabular}{|c|c|c|}
\hline Universität & EH-Kurs & Organisiert durch: \\
\hline \multicolumn{3}{|c|}{ Staatliche Medizinische Universitäten in Deutschland } \\
\hline RWTH Aachen & $\mathrm{Ja}$ & Universitär/Curriculär \\
\hline Universität Augsburg & - & * \\
\hline Humboldt-Universität zu Berlin & - & * \\
\hline Ruhr-Universität Bochum & $\mathrm{Ja}$ & Studentisch \\
\hline Friedrich-Wilhelms-Universität Bonn & - & * \\
\hline Technische Universität Dresden & $\mathrm{Ja}$ & Studentisch: AG EH-Med \\
\hline Heinrich-Heine-Universität Düsseldorf & $\mathrm{Ja}$ & Kooperation \\
\hline Friedrich-Alexander-Universität Erlangen-Nürnberg & $\mathrm{Ja}$ & Universitätsklinikum \\
\hline Universität Duisburg-Essen & $\mathrm{Ja}$ & Kooperation \\
\hline Goethe-Universität Frankfurt am Main & $\mathrm{Ja}$ & Kooperation \\
\hline Albert-Ludwigs-Universität Freiburg & - & * \\
\hline Justus-Liebig-Universität Gießen & $\mathrm{Ja}$ & Studentisch: AG EH-Med \\
\hline Georg-August-Universität Göttingen & $\mathrm{Ja}$ & Studentisch \\
\hline Ernst-Moritz-Arndt-Universität Greifswald & $\mathrm{Ja}$ & Studentisch: AG EH-Med \\
\hline Martin-Luther-Universität Halle-Wittenberg & $\mathrm{Ja}$ & Universitätsklinikum \\
\hline Universität Hamburg & $\mathrm{Ja}$ & Studentisch - DRK \\
\hline Medizinische Hochschule Hannover & - & * \\
\hline Ruprecht-Karls-Universität Heidelberg & $\mathrm{Ja}$ & Kooperation \\
\hline Universität des Saarlandes Homburg & $\mathrm{Ja}$ & Studentisch - Malteser \\
\hline Friedrich-Schiller-Universität Jena & - & nicht ersichtlich \\
\hline Christian-Albrechts-Universität Kiel & - & * \\
\hline Universität Köln & $\mathrm{Ja}$ & Universität \\
\hline Universität Leipzig & $\mathrm{Ja}$ & Studentisch: AG EH-Med \\
\hline
\end{tabular}




\begin{tabular}{|c|c|c|}
\hline Universität Lübeck & $\mathrm{Ja}$ & Studentisch: AG EH-Med \\
\hline Otto-von-Guericke-Universität Magdeburg & - & * \\
\hline Medizinische Fakultät Mannheim & $\mathrm{Ja}$ & Universität: Wahlfach \\
\hline Johannes-Gutenberg-Universität Mainz & $\mathrm{Ja}$ & Kooperation \\
\hline Philipps-Universität Marburg & - & * \\
\hline Ludwig-Maximilians-Universität München & - & * \\
\hline Technische Universität München & - & \\
\hline Westfälische Wilhelms-Universität Münster & $\mathrm{Ja}$ & Studentisch: AGN \\
\hline Carl-von-Ossietzky-Universität Oldenburg & - & * \\
\hline Universität Regensburg & $\mathrm{Ja}$ & Studentisch \\
\hline Universität Rostock & $\mathrm{Ja}$ & Studentisch: AG EH-Med \\
\hline Eberhard-Karls-Universität Tübingen & $\mathrm{Ja}$ & Kooperation \\
\hline Universität UIm & - & * \\
\hline Julius-Maximilians-Universität Würzburg & - & * \\
\hline \multicolumn{3}{|c|}{ Private Medizinische Universitäten in Deutschland: } \\
\hline Universität Witten/Herdecke & $\mathrm{Ja}$ & Studentisch \\
\hline Medizinische Hochschule Brandenburg & - & * \\
\hline Paracelsus-Universität Standort Nürnberg & - & * \\
\hline \multirow[t]{2}{*}{ Kassel School of Medicine } & - & * \\
\hline & Anzahl & \\
\hline Gesamtzahl Universitäten & 41 & \\
\hline Erste-Hilfe-Kurs angeboten & 24 & \\
\hline Von der Universität angeboten & 3 & \\
\hline Von Studierenden selbst angeboten & 13 & \\
\hline Vom Universitätsklinikum angeboten & 2 & \\
\hline In Kooperation Universität - Studierende & 6 & \\
\hline
\end{tabular}

Tabelle 1: Erste-Hilfe-Kurse für Vorklinikstudierende an den medizinischen Fakultäten. Selbstrecherche auf den Internetseiten der jeweiligen Universitäten, Stand November 2019. *Keine Angaben auf der Universitäten Internetseite verfügbar.

Davon ist bei nur zwei Universitäten (Aachen ${ }^{66}$, Köln) der Kurs curricular eingebunden und somit für die Studierenden verpflichtend. Bei den übrigen handelt es sich meist um studentische Initiativen, in einigen Fällen auch um Kooperationen mit der Universität oder den dazugehörigen Skills Labs der Universität. Diese Kurse sind 
speziell auf Medizinstudierende zugeschnitten und garantieren eine „zielgruppenspezifische Ausbildung mit einer Steigerung des Identifikationsgrads ${ }^{34 “}$, wie Wiese et al. ${ }^{34}$ empfehlen.

Die Rheinisch-Westfälische Technische Hochschule Aachen hat im Zuge der Umstellung zum Reformstudiengang Medizin auch die notfallmedizinische Ausbildung der Studierenden angepasst. Hier starten die Studierenden in ihrem ersten Semester mit einer Einführung in die Notfallmedizin. ${ }^{66}$ Dort lernen sie theoretisch und praktisch notfallmedizinische Basismaßnahmen wie bspw. BLS und die Nutzung eines AED. ${ }^{66}$ Im Verlauf des Studiums werden die Kenntnisse durch aufeinander folgende Kurse immer weiter bis zum Advanced Life Support ausgebaut. ${ }^{66}$ Dieses im Jahr 2002 eingeführte System war deutschlandweit einzigartig und fortschrittlich in der notfallmedizinischen Ausbildung Medizinstudierender. ${ }^{66}$

Als Beispiel für einen studentisch geleiteten, multizentrischen Notfallkurs speziell für Vorklinikstudierende kann das Kurskonzept der Arbeitsgemeinschaft Erste Hilfe und Notfallkunde für Medizinstudierende e.V. (AGEHMED) aufgeführt werden. Dieses „wurde erstmals 1996 an der Freien Universität Berlin [...] aufgrund eines unzureichenden Angebots von zielgruppenorientierten Notfallkursen durchgeführtt ${ }^{67 \text { " und }}$ hat sich mehrfach bewährt. ${ }^{67}$ Auch dieser Kurs, der von Studierenden für Studierende konzipiert wurde, besteht aus den „Grundlagen der Ersten Hilfe sowie erweiterten ärztlichen und rettungsdienstlichen Maßnahmen der notfallmedizinischen Versorgung67“. Im Rahmen des Peer Assisted Learning (PAL) „wurde ein fünfstufiges, supervidiertes Qualifizierungskonzept etabliert, welches den Medizinstudierenden unabhängig von Vorkenntnissen ermöglicht, eine Ausbilderbefähigung zu erlan-

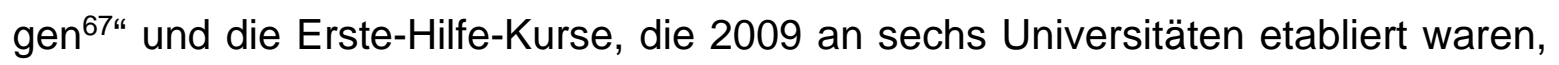
zu unterrichten. Das multizentrische Kurskonzept, welches zum Untersuchungszeitpunkt der Studie von lblher et al. ${ }^{67}$ bereits seit sieben Jahren bestand, zeigte eine „kontinuierlich hohe Projektakzeptanz [...] seitens der Kursteilnehmer ${ }^{67 “ .}$ 


\subsubsection{Wissensstand und praktische Kompetenzen der Medizinstudierenden}

Eine Studie von Ammirati et al. ${ }^{68}$ an Medizinstudierenden im ersten Studienjahr in Frankreich zeigt, dass nur 4,5\% der Teilnehmenden im Rahmen eines Herz-Kreislauf-Stillstands den AED einsetzten. 30\% legten die Patient:innen zunächst in die stabile Seitenlage, bevor sie mit der Thoraxkompression begannen. ${ }^{68}$

Diese Studie ist kein Einzelfall: viele vorangegangene Studien zeigen mehrfach, dass das Basiswissen in Erster Hilfe und die praktischen Kompetenzen in der Herzlungenwiederbelebung von Medizinstudierenden nicht ausreichen, um adäquat Notfälle bewältigen zu können. ${ }^{69-74}$ Selbst wenn die Studierenden ein halbes Jahr vorher einen BLS-Kurs im Rahmen des Führerscheins absolviert haben, zeigte sich ein enttäuschendes Ergebnis und kein Unterschied zu Studierenden, die zuvor keinen BLSKurs besucht haben. ${ }^{71}$

Auch in Deutschland fiel auf, dass das notfallmedizinische Training in der Ausbildung von Medizinstudierenden unstrukturiert und nicht ausreichend ist. ${ }^{63}$ Somit empfehlen Expert:innen bereits eine frühzeitige Etablierung eines BLS-Kurses, um notfallmedizinische Basiskompetenzen zu erlernen. ${ }^{71,75,76}$ Seidman et al. ${ }^{77}$ vermuten, dass eine frühe Ausbildung klinisch-praktischer Fähigkeiten den Studierenden in den klinischen Studienabschnitten ermöglicht, schneller ein höheres Maß an Vertrauen zu erlangen, als wenn das Training erst innerhalb des klinischen Studiums begonnen wird. Darüber hinaus bietet eine frühzeitige standardisierte Schulung aller Studierenden die Möglichkeit, eine Grundlage für die Ausbildung von klinischen Fertigkeiten zu schaffen. ${ }^{77}$

Störmann et al. ${ }^{78}$ fanden heraus, dass "die Leistungen von Studierenden im Praktischen Jahr bei der Durchführung klinisch-praktischer Kompetenzen im Rahmen einer mündlich-praktischen Prüfung [...] Raum für Verbesserung [bieten]. Nahezu zwei Drittel der Studierenden zeigen lediglich eine befriedigende oder ausreichende Leistung; jeder zehnte Teilnehmer versagt in der Prüfung. Dabei überschätzt knapp die Hälfte der Studierenden die eigene Leistung. ${ }^{78 “}$

Eine Studie von Dafsari et al. ${ }^{79}$ zeigt, dass auch die Studierenden den klinisch-praktischen Disziplinen durchweg mehr praktische Unterrichtszeit zugestehen würden, 
als den klinisch-theoretischen und dort auch die Lernmotivation und der Nutzen im ärztlichen Alltag signifikant höher eingestuft wurde. Es zeigte sich, dass parallel zum Studium verlaufende Skill Trainings eine gute Vorbereitung auf klinische Praktika darstellen und die Lernfähigkeiten in diesen verbessern. ${ }^{80}$ Seale et al. ${ }^{81}$ konnten in ihrer Studie darstellen, das Studierende praktisch-klinische Fähigkeiten im ersten Jahr des Medizinstudiums begrüßen würden und diese die Lernmotivation und Bereitschaft der Studierenden zur Durchführung der gelehrten Techniken steigert. Die Studierenden fordern insgesamt mehr Übungs- und Feedbackmöglichkeiten durch geschulte Tutor:innen, bei vorwiegend auf Praxis basierenden Lehrmodulen. ${ }^{81,82} \mathrm{Zu}-$ dem wünschen sie sich eine häufigere und intensivere notfallmedizinische Ausbildung. ${ }^{69}$

\subsection{Standardisierte Aus- und Weiterbildung in der Notfallmedizin}

Sowohl ärztliches als auch rettungsdienstliches Personal hat die Möglichkeit, sich in standardisierten Kursen, die sich an evidenzbasierter Medizin und gängigen Leitlinien orientieren, fortzubilden. Zu diesen Kursformaten zählen u.a. Kurse der ERC, der AHA oder des amerikanischen Rettungsdienst-Berufsverbandes, der National Association of Emergency Medical Technicians (NAEMT), in denen "manuelle Fertigkeiten, Algorithmen und Entscheidungshilfen gelehrt83" werden, die für die Abarbeitung präklinischer Notfälle hilfreich sind. ${ }^{84-86}$ „Ein wesentlicher Aspekt ist dabei das strukturierte Erkennen und Behandeln lebensbedrohlicher Zustände. ${ }^{87 “}$

Die Forderung von Expert:innen „EBM-Leitlinien und konzertierte[n] Empfehlungen nationaler/europäischer oder international führender Fachgesellschaften [...] deutlicher in den Mittelpunkt zu stellen“ wird immer lauter, da eine „einheitliche Herangehensweise [...] einen Gewinn für den Patienten ${ }^{42 “}$ darstellt. ${ }^{88-90}$ Zusätzlich wird eine kontinuierliche Fortbildung gefordert, „die auf einem standardisierten medizinischen und didaktischen Konzept beruhen, um eine vergleichbare und hohe Qualität ${ }^{88 \text { " }} \mathrm{zu}$ erreichen. ${ }^{84,90}$ 


\subsubsection{ABCDE-Schema}

In der präklinischen Notfallmedizin wird zur strukturierten Erstuntersuchung der $\mathrm{Pa}$ tient:innen vorwiegend das ABCDE-Schema verwendet. Als standardisierter Algorithmus hat sich dieses Schema seit mehreren Jahren bewährt, ${ }^{91}$ um „den Zustand des Patienten schnell zu erfassen, ohne dabei lebensbedrohliche Zustände zu übersehen ${ }^{92 “ . ~ D a b e i ~ s t e h e n ~ d i e ~ B u c h s t a b e n ~ f u ̈ r ~ d i e ~ e i n z e l n e ~ A b s c h n i t t e ~ d e r ~ U n t e r s u-~}$ chung an Patient:innen, um lebensbedrohliche Situationen schnell zu erkennen und zielführend zu behandeln. A steht dabei für Airway, B für Breathing, $C$ für Circulation, D für Disability und E für Environment/Exposure. ${ }^{91,92}$ Die strukturierte Untersuchung ist für Erwachsene und Kinder nutzbar und kann von jeder Person ohne nötige Hilfsmittel angewendet werden. ${ }^{91}$

\begin{tabular}{|l|l|l|}
\hline A & $\begin{array}{l}\text { Airway } \\
\text { (Atemwege) }\end{array}$ & $\begin{array}{l}\text { Atemwege überprüfen } \\
\text { Ggf. Atemwege freimachen } \\
\text { Ggf. Kopf überstrecken }\end{array}$ \\
\hline B & $\begin{array}{l}\text { Breathing } \\
\text { (Atmung, Beatmung) }\end{array}$ & $\begin{array}{l}\text { Atmung überprüfen } \\
\text { Ggf. O2-Gabe } \\
\text { Ggf. Beatmung }\end{array}$ \\
\hline C & $\begin{array}{l}\text { Circulation } \\
\text { (Kreislauf) }\end{array}$ & Kreislauf überprüfen \\
\hline D & $\begin{array}{l}\text { Disability } \\
\text { (neurologisches Defizit) }\end{array}$ & $\begin{array}{l}\text { Oewusstsein überprüfen } \\
\text { Orientierende neurologische Untersuchung }\end{array}$ \\
\hline E & $\begin{array}{l}\text { Environment/Exposure } \\
\text { (weitere Untersuchung) }\end{array}$ & Patient entkleiden und orientierend untersuchen \\
\hline
\end{tabular}

Tabelle 2: ABCDE-Schema nach Ziegenfuß et al. ${ }^{93}$

Medizinische Algorithmen repräsentieren anerkannte Richtlinien und bilden hieraus einheitliche Behandlungsleitlinien. Sie dienen dazu, schnell und zuverlässig standardisierte Programme abarbeiten zu können: „Dies erleichtert die Zusammenarbeit, strukturiert das präklinische und das klinische Vorgehen und trägt wesentlich zur Standardisierung [...] bei. ${ }^{94 “}$ Algorithmen zeichnen einen strukturierten Lösungsweg 
auf und geben vor allem unter Zeitdruck Sicherheit und Rückhalt. Aufgrund dessen verbessert die Nutzung des ABCDE-Schemas das Outcome der Patient:innen und sollte gehäuft eingesetzt werden. ${ }^{91}$

Die gängigen Erste-Hilfe-Kurse orientieren sich wie oben bereits beschrieben überwiegend an der Rettungskette und gliedern dort die erforderlichen Notfallmaßnahmen unter. Das ABCDE-Schema spielt bisher in den Laien-Kursen keine elementare Rolle.

\subsection{Ziele der Arbeit}

Es stellt sich die Frage, welche notfallmedizinischen Basiskompetenzen Vorklinikstudierende beherrschen und ob diese ausreichen, um Notfallsituationen als Ersthelfende adäquat zu bewältigen. Des Weiteren ergibt sich aus dem vorangehenden Abschnitt die Frage, ob durch einen speziell auf Medizinstudierende zugeschnittenen Erste-Hilfe-Kurs anhand des ABCDE-Schemas die notfallmedizinischen Basiskompetenzen gesteigert werden können.

Daher sind die Ziele dieser Arbeit die Durchführung einer Statusanalyse des vorhandenen Erste-Hilfe-Wissens und der dazugehörigen Kompetenzen bei den Studierenden im vorklinischen Studienabschnitt sowie die Entwicklung und Etablierung eines Erste-Hilfe-Kurses für Vorklinikstudierende. Weiterhin erfolgt die Erfolgskontrolle nach Implementierung des Kurses und die kritische Auseinandersetzung mit der Frage, inwieweit die universitären Strukturen verbessert werden können, um die Studierenden auf Notfallsituationen in der Klinik ausreichend vorzubereiten. 


\section{Fragestellungen}

Aufgrund der zuvor genannten Problematik werden folgende Forschungsfragen im Rahmen der vorliegenden Arbeit untersucht:

1. Welche notfallmedizinischen Basiskompetenzen besitzen die Studierenden im vorklinischen Studienabschnitt der Goethe-Universität Frankfurt vor der Absolvierung des Erste-Hilfe-Kurses?

2. Kann ein Erste-Hilfe-Kurs basierend auf dem ABCDE-Schema konzipiert und durchgeführt werden?

3. Können durch einen Erste-Hilfe-Kurs basierend auf dem ABCDE-Schema die notfallmedizinischen Kompetenzen der Studierenden verbessert werden? 


\section{Material und Methoden}

\subsection{Konzeption des Erste-Hilfe-Kurses}

Die Idee zur Konzeption und Etablierung eines Erste-Hilfe-Kurses für Medizinstudierende in Frankfurt entstand innerhalb der Fachschaft Medizin, da viele Studierende die Qualität, der in Frankfurt durch verschiedenste Anbieter angebotenen Kurse für Erste Hilfe bemängelten.

Die Zielsetzung der sich daraufhin entwickelnden Arbeitsgruppe, bestehend aus Medizinstudierenden der Fachschaft und Mitarbeitenden des Frankfurter Interdisziplinären Simulationstrainings (FIneST), war es, einen qualitativ hochwertigen Kurs speziell für Medizinstudierende zu möglichst geringen Teilnahmekosten anzubieten. Die Konzeption des Erste-Hilfe-Kurses erfolgte in mehreren Schritten, die im Folgenden dargestellt sind und den sechs Schritten des Kernzyklus zur Curriculumsentwicklung entsprechen (Abb. 3).

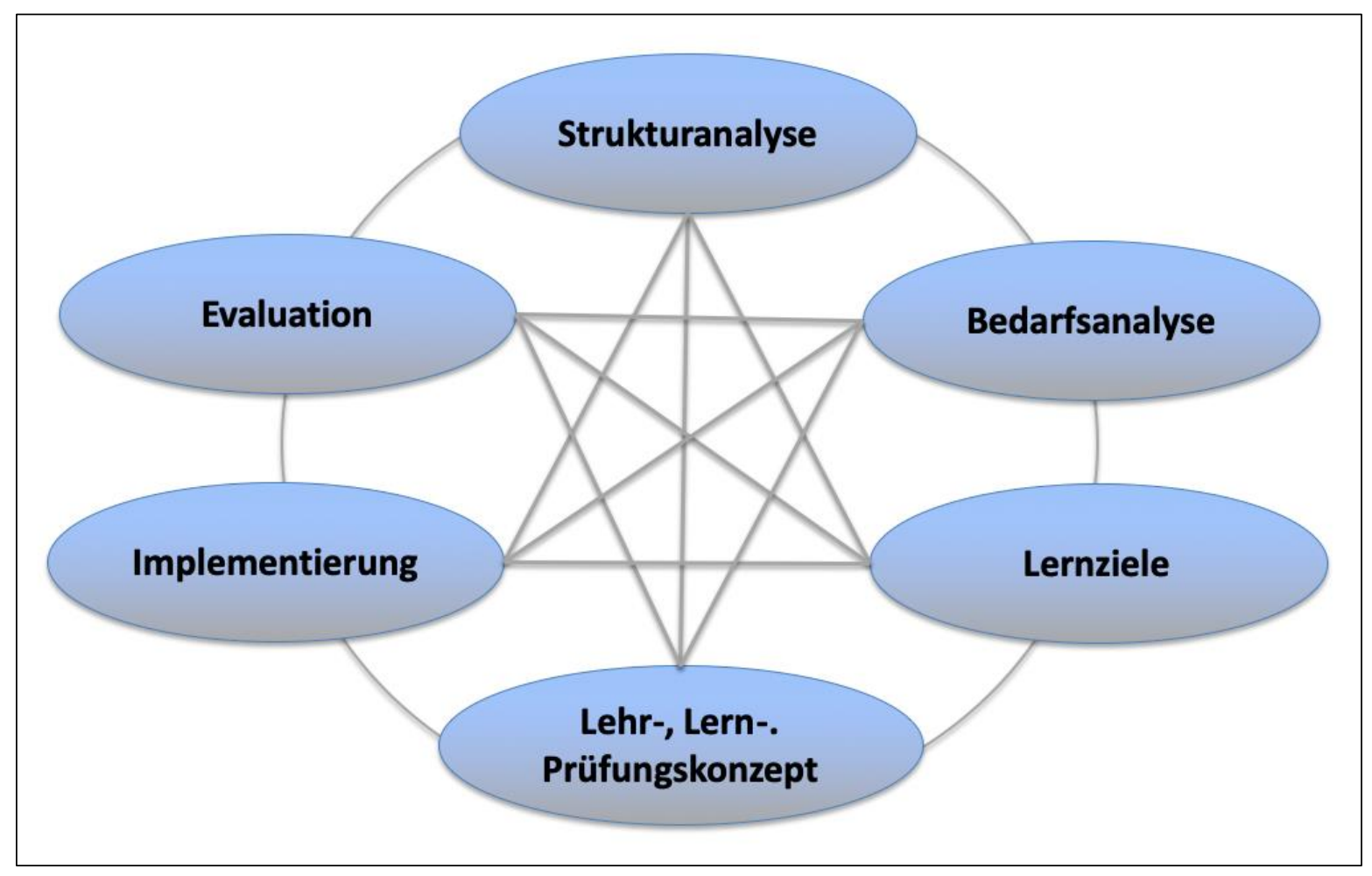

Abbildung 5: Arbeitsschritte zur Curriculumsentwicklung basierend auf den 6-Schritten nach Kern. ${ }^{95}$ 
Zunächst analysierte die Arbeitsgruppe im Rahmen einer Strukturanalyse die rechtlichen Grundlagen und Vorgaben der Inhalte, Kursstrukturen, Gruppengröße, etc. zu Erste-Hilfe-Kursen in Deutschland. Diese wurden in der Einleitung vorgestellt. Anschließend erfolgte die Bedarfsanalyse. Hier zeigte sich wie bereits erläutert, dass es zwar eine Vielzahl von Erste-Hilfe-Kursen gab, die von verschiedensten Anbietern in der Rhein-Main-Region angeboten werden, jedoch konnte keiner in Hinblick auf Gruppengröße, Anteil praktischer Übungen, vermittelte Lernziele, etc. die Kriterien eines adäquaten Kurses für Medizinstudierende erfüllen.

\subsubsection{Lernziele}

\subsubsection{Lehr-/Lernkonzept}

Das Besondere an dem Projekt war die inhaltlich-didaktische Ausgestaltung des Kurses, der sich in wesentlichen Punkten von den bisher angebotenen Kursformaten unterscheidet. Wie Soar et al. ${ }^{96}$ feststellten, soll „das Training [...] auf die Bedürfnisseder unterschiedlichen Lerntypen und -stile zugeschnitten sein, um das Erwerben und Behalten von Wissen sowie Fertigkeiten der Wiederbelebung sicherzustellen. ${ }^{96 "}$ Es zeigte sich als hilfreich, die Motivation der Studierenden und die klinische Relevanz der einzelnen, zu vermittelnden Themen im Voraus zu beleuchten. ${ }^{97}$

Aus diesem Grund liegt die Schwerpunktsetzung dieses Erste-Hilfe-Kurses auf dem algorithmenorientierten Lernen, welches für Medizinstudierende im klinischen Studienabschnitt eine wesentliche Grundlage für das Lernen darstellt, bis dato jedoch nicht in Erste-Hilfe-Kursen implementiert wurde. Hierzu wurden die zu erlernenden theoretischen und praktischen Lernziele in strukturierter Reihenfolge basierend auf dem ABCDE-Schema eingegliedert. So können die Studierenden jede erlernte Maßnahme inhaltlich-sinnvoll im Zeitverlauf einer Notfallsituation nachvollziehen und werden gleichzeitig direkt mit der klinischen Praxis vertraut.

Zudem integriert das Kurskonzept basierend auf den didaktischen Konzepten der Erwachsenenbildung das vorhandene Vorwissen der Medizinstudierenden aus Anatomie, Biochemie und Physiologie. ${ }^{98-101}$ Diese werden aktiv wiederholt, mit neuem 
Wissen verknüpft und in die konkrete Problemlösung eingebracht, um den Lernprozess zu verbessern. ${ }^{99-101}$ Mit dieser Form des kognitiven Lernens wird versucht, durch Verknüpfung von vorhandenem Wissen das neu Erlernte leichter zu behalten. ${ }^{102}$ Durch die von der Arbeitsgruppe gebildeten Handlungspläne und die „Verknüpfung einzelner Informationen zu Schlüssen ergeben sich Lernschritte ${ }^{102 “}$, die die Studierenden möglichst effektiv und in kurzer Zeit zum Lernerfolg führen sollen. Mit dem Wissen der einzelnen Lerntypen wurde versucht, ein möglichst breites methodisch-didaktisches Kursprogramm zu etablieren.

\begin{tabular}{|c|c|c|}
\hline Lerntyp & Eigenschaften & Lernhilfen \\
\hline $\begin{array}{l}\text { visueller } \\
\text { Lerntyp }\end{array}$ & $\begin{array}{l}\text { - liest gerne } \\
\text { - schaut Illustrationen und Grafiken an } \\
\text { - arbeitet gern mit schriftlichen Unterlagen }\end{array}$ & $\begin{array}{l}\text { Bücher, Skizzen, Bilder, } \\
\text { Lernposter, Videos, } \\
\text { Lernkarteien, (selbst er- } \\
\text { stellte) Mindmaps, } \\
\text { Schaubilder, Tabellen }\end{array}$ \\
\hline $\begin{array}{l}\text { auditiver } \\
\text { Lerntyp }\end{array}$ & $\begin{array}{l}\text { - kann leicht gehörte Informationen aufnehmen } \\
\text { • kann mündliche Erklärungen aufnehmen } \\
\text { • kann gut auswendig lernen }\end{array}$ & $\begin{array}{l}\text { Lern-Audioprogramme, } \\
\text { Gespräche, Vorträge }\end{array}$ \\
\hline $\begin{array}{l}\text { haptischer } \\
\text { Lerntyp }\end{array}$ & $\begin{array}{l}\text { • „Learning-by-doing“ } \\
\text { • führt Handlungsabläufe selber durch } \\
\text { • Erklärungen „begreift“ er } \\
\text { • probiert gern aus (Experimente) }\end{array}$ & $\begin{array}{l}\text { Imitation, Gruppenaktivi- } \\
\text { täten, Rollenspiele, Si- } \\
\text { mulationen }\end{array}$ \\
\hline $\begin{array}{l}\text { kommunikativer } \\
\text { Lerntyp }\end{array}$ & $\begin{array}{l}\text { - gelangt durch Gespräche und Diskussionen zum } \\
\text { größten Lernerfolg } \\
\text { - muss Erklärungen durchsprechen, diskutieren } \\
\text { - Position des Fragenden und des Erklärenden }\end{array}$ & $\begin{array}{l}\text { Dialoge, Diskussionen, } \\
\text { Lerngruppen, Rollen- } \\
\text { spiele, Frage-Antwort- } \\
\text { Spiele }\end{array}$ \\
\hline
\end{tabular}

Tabelle 3: Die verschiedenen Lerntypen nach Ahlemann et al. ${ }^{102}$

Um die Struktur der Rettungskette und die Gliederung des ABCDE-Schemas zu verdeutlichen, wurde während des Kurses ein Schaubild auf der zur Verfügung stehenden Tafel erarbeitet. Im Rahmen einer PowerPoint-Präsentation wurden Bilder und 
Videos eingesetzt, sowie Vorträge durch Peer-Dozierende gehalten. Zusätzlich wurden komplizierte Sachverhalte in Form von Gruppenarbeiten erarbeitet und praktische Maßnahmen geübt.

Ein besonderer Fokus des Konzeptes liegt auf den praktischen Übungen der HLW. In den existierenden Erste-Hilfe-Kurskonzepten werden diese meist nur sehr kurz thematisiert, jedoch sind das Erkennen eines Herzkreislaufstillstandes und der direkte Beginn der Reanimation für das Überleben und das Outcome der Betroffenen von entscheidender Bedeutung. Aus diesem Grund wurde das CPR-Training an professionellen Reanimations-Puppen mit Feedback-Systemen geübt. Es konnte gezeigt werden, dass „Hilfsmittel, die CPR-Kommandos oder -Rückmeldung geben, [...] das Erlernen und Behalten von CPR-Fertigkeiten [verbessern]. .96" Insbesondere Feedback-Systeme in Echtzeit scheinen in Trainings hilfreich zu sein und das Bewusstsein für die klinische Wichtigkeit zu verbessern. ${ }^{103,104}$ Die Kombination aus Instruktor:innen-Feedback und integriertem Feedback-System stellte sich als nützlich heraus und wurde aus diesem Grund in das Kurssystem des Erste-Hilfe-Kurses etabliert. ${ }^{105}$

Ein weiteres Anliegen bei der Konzipierung war die Einbindung des Nationalen Kompetenzbasierten Lernzielkataloges Medizin (NKLM). Eine Integration der geforderten Basiskompetenzen im Kapitel „Notfallmedizin“ in den Kurs wurde angestrebt.

\begin{tabular}{|c|c|c|c|c|c|c|}
\hline Position & Bezeichnung & GK & BK & PJ K & Wb K & WK \\
\hline 17.1.3.1 & $\begin{array}{l}\text { Die Maßnahmen zum Eigenschutz, } \\
\text { zum Schutz von Patientinnen und } \\
\text { Patienten und weiteren Anwesenden } \\
\text { an einer Einsatzstelle erläutern und } \\
\text { anwenden. }\end{array}$ & & 1 & 2 & 2 & \\
\hline 17.1.3.2 & $\begin{array}{l}\text { die Rettung von Patientinnen und } \mathrm{Pa} \text { - } \\
\text { tienten aus einem Gefahrenbereich } \\
\text { durchführen. }\end{array}$ & & $3 a$ & $3 a$ & $3 a$ & \\
\hline
\end{tabular}




\begin{tabular}{|c|c|c|c|c|}
\hline 17.5.1.1 & $\begin{array}{l}\text { unterschiedliche Lagerungsarten im } \\
\text { Notfall und ihre Indikationen benen- } \\
\text { nen sowie die Lagerung bei gegebe- } \\
\text { ner Indikation demonstrieren. }\end{array}$ & $3 a$ & $3 b$ & $3 b$ \\
\hline 17.5.2.1 & $\begin{array}{l}\text { den Basic Life Support-Algorithmus } \\
\text { anwenden }\end{array}$ & $3 a$ & $3 b$ & $3 b$ \\
\hline 17.6.1.1 & $\begin{array}{l}\text { Bewusstseinsstörungen und neurolo- } \\
\text { gische Defizite aus notfallmedizini- } \\
\text { scher Sicht erläutern. }\end{array}$ & 1 & 2 & 2 \\
\hline 17.6.1.2 & $\begin{array}{l}\text { die akute Atemnot aus notfallmedizi- } \\
\text { nischer Sicht erläutern. }\end{array}$ & 1 & 2 & 2 \\
\hline 17.6.1.3 & $\begin{array}{l}\text { den Brustschmerz aus notfallmedizi- } \\
\text { nischer Sicht erläutern. }\end{array}$ & 1 & 2 & 2 \\
\hline 17.6.1.4 & $\begin{array}{l}\text { Blutungen aus notfallmedizinischer } \\
\text { Sicht erläutern. }\end{array}$ & 1 & 2 & 2 \\
\hline 17.6.1.5 & $\begin{array}{l}\text { den Schock aus notfallmedizinischer } \\
\text { Sicht erläutern. }\end{array}$ & 1 & 2 & 2 \\
\hline 17.6.1.7 & $\begin{array}{l}\text { den abdominalen Schmerz aus not- } \\
\text { fallmedizinischer Sicht erläutern. }\end{array}$ & 1 & 2 & 2 \\
\hline 17.6.1.8 & $\begin{array}{l}\text { akute psychische Störungen aus not- } \\
\text { fallmedizinischer Sicht benennen und } \\
\text { erkennen. }\end{array}$ & 1 & 2 & 2 \\
\hline 17.6.1.10 & $\begin{array}{l}\text { Kolikschmerzen erkennen, deren Dif- } \\
\text { ferenzialdiagnosen benennen und } \\
\text { Beispiele für dringliche Handlungsin- } \\
\text { dikationen aufführen. }\end{array}$ & 1 & 2 & 2 \\
\hline
\end{tabular}

Tabelle 4: Notfallmedizinische Lernziele nach dem NKLM. ${ }^{106} 1$ = Faktenwissen, 2 = Handlungs- und Begründungswissen, 3 = Handlungskompetenz: $3 \mathrm{a}=$ unter Anleitung selbst durchführen und demonstrieren. $3 \mathrm{~b}=$ selbstständig und situationsadäquat in Kenntnis der Konsequenzen durchführen. GK = Grundlagenkompetenz, $\mathrm{BK}=$ Ärztliche Basiskompetenzen für die Ausbildung mit unmittelbarem Patientenbezug, PJ K = PJ-Kompetenz, Wb K = Ärztliche Approbation und Weiterbildungskompetenz, WK = Wissenschaftskompetenz 
Insbesondere folgende theoretische und praktische Inhalte, die im Lernzielkatalog gefordert werden, wurden in das Kurskonzept eingegliedert:

\begin{tabular}{|l|l|}
\hline Lagerungsarten & $\begin{array}{l}\text { Stabile Seitenlage, Kopf-Hoch-Lagerung, Oberkör- } \\
\text { per-Hoch-Lagerung, Bauch-Schon-Lagerung, } \\
\text { Schock-Lagerung, Links-Seitenlage }\end{array}$ \\
\hline Basic Life Support & $\begin{array}{l}\text { BLS-Algorithmus, Herzdruckmassage; Kardiover- } \\
\text { sion, Defibrillation, Atemwege frei machen (Kopf } \\
\text { überstrecken, Kinn anheben) }\end{array}$ \\
\hline Bewusstseinsstörungen \\
und neurologische Defizite & $\begin{array}{l}\text { Hypo-/Hyperglykämie, Schädel-Hirn-Trauma, } \\
\text { Schlaganfall, Krampfanfall, Intoxikation }\end{array}$ \\
\hline akute Atemnot & $\begin{array}{l}\text { AKS, Lungenembolie, Asthma/exazerbierte COPD, } \\
\text { Lungenödem, (Spannungs-) Pneumothorax, } \\
\text { Fremdkörper-Aspiration, Hyperventilation }\end{array}$ \\
\hline Blutungen & $\begin{array}{l}\text { AKS, Lungenembolie, Aortendissektion, } \\
\text { (Spannungs-) Pneumothorax, Panikattacke }\end{array}$ \\
\hline Schock & $\begin{array}{l}\text { Gastrointestinale Blutungen, Trauma [...] } \\
\text { hypovolämisch, kardial, anaphylaktisch, septisch, } \\
\text { neurogen }\end{array}$ \\
\hline Abdominalen Schmerz & $\begin{array}{l}\text { Aortendissektion, Perforation von Hohlorganen, } \\
\text { Ileus, Koliken, entzündliche Prozesse (z.B. Appen- } \\
\text { dizitis), extrauterine Gravidität, Trauma }\end{array}$ \\
\hline Psychische Störung & $\begin{array}{l}\text { Panikattacke, akute Eigen- oder Fremdgefährdung, } \\
\text { Parasuizidalität/Suizidandrohung/Suizid, Brust- } \\
\text { schmerz bei Herzangst, Alkoholintoxikation [...] }\end{array}$ \\
\hline
\end{tabular}

Tabelle 5: Integrierte Kursinhalte des Erste-Hilfe-Kurses basierend auf den Lernzielen im NKLM. ${ }^{106}$ 


\subsubsection{Kursplan}

Im Vorfeld des Erste-Hilfe-Kurses waren einige Vorarbeiten nötig: Eine aus Studierenden bestehende Arbeitsgruppe entwickelte das Kurskonzept, mit genauem Ablaufplan des Kurses sowie einem festgelegten Curriculum. Dieses basiert auf den aktuellen Leitlinien der AHA und der ERC und enthält die gängigen Erste-Hilfe-Kursinhalte. Die gesamte Kurslänge beträgt, wie seit dem 01. April 2015 vorgeschrieben, 9 Unterrichtseinheiten à 45 Minuten, eine kurze Übersicht ist in der folgenden Abbildung dargestellt.

\begin{tabular}{|c|c|}
\hline 1. UE & Erstes Glied der Rettungskette \\
\hline 2. UE & Zweites Glied der Rettungskette: Airway, Breathing \\
\hline 3. UE & Zweites Glied der Rettungskette: Herz-Lungen-Wiederbelebung \\
\hline 4. UE & Zweites Glied der Rettungskette: Herz-Lungen-Wiederbelebung \\
\hline 5. UE & $\begin{array}{l}\text { Zweites Glied der Rettungskette: } \\
\text { Bewusstlosigkeit erkennen, Seitenlage, Helmabnahme }\end{array}$ \\
\hline 6. UE & Zweites Glied der Rettungskette: Circulation \\
\hline 7. UE & Zweites Glied der Rettungskette: Disability, Environment/Exposure \\
\hline 8. UE & Drittes Glied der Rettungskette \\
\hline 9. UE & Viertes und fünftes Glied der Rettungskette \\
\hline
\end{tabular}

Tabelle 6: Die 9 Unterrichtseinheiten des Erste-Hilfe-Kurses.

Innerhalb der Unterrichtseinheiten gibt es klare Lernzieldefinitionen für die zu vermittelnden Maßnahmen und theoretischen Inhalte. Zusätzlich wurde darauf geachtet, die praktischen Maßnahmen zu priorisieren und dafür innerhalb des Kurses ausreichend Zeit zu schaffen.

\subsubsection{Peer-Dozierende}

„Peer" kommt aus dem Englischen und bedeutet „der Gleichrangige“. Bei Peer-Dozierenden handelt es sich um Dozierende, die keine professionellen Lehrer:innen sind, sondern aus einer ähnlichen sozialen Gruppe stammen, beispielsweise Studierende, die sich gegenseitig helfen. ${ }^{107}$ Peer-geleitete Kurse sind in Deutschland 
längst keine Seltenheit mehr und fest in der studentischen, medizinischen Lehre implementiert. ${ }^{108,109}$ Der Anstieg des PAL ist u.a. durch den Anstieg der Studierendenzahl, begrenzte Lehrmittel und den Ansprüchen an Lehre, Leistungsbewertung und Kompetenzen der Medizinstudierenden zu erklären. ${ }^{110}$ Mehrere Studien zeigten, dass Peer-Dozierende nicht nur für Schüler:innen, ${ }^{111-113}$ sondern auch für Medizinstudierende ${ }^{114-122}$ genauso effektiv in der Vermittlung der Lernziele in der Notfallmedizin, insbesondere im BLS-Training sind, wie professionelle Instruktor:innen. Vor allem bei Kursen, die aufgrund kleiner Gruppengrößen und benötigter, intensiver Betreuung auf eine hohe Anzahl von Personal angewiesen sind, hat sich der Einsatz von Peer-Dozierenden bewährt. ${ }^{116}$ Harvey et al. ${ }^{117}$ zeigten in einer groß angelegten Studie über 15 Jahre, dass Peer-geleitete BLS-Kurse herausragende Ergebnisse erzielen konnten und die Teilnehmenden sehr zufrieden waren. Die Peer-Instruktor:innen waren kompetent, zeitlich flexibel und insgesamt kostengünstigere Arbeitskräfte, als professionelles Klinikpersonal. ${ }^{117}$ In der Studie von Hughes et al. ${ }^{118}$ wurde beleuchtet, dass Medizinstudierende aus höheren Semestern mit klinischer Vorbildung geeignet sind, jüngere Semester auch in fortgeschrittenen Reanimationsmaßnahmen zu unterrichten. Auch die Peer-Gruppe profitiert in dieser Rolle, lernt dazu und wird motiviert, die Lehr-Rolle in der weiteren klinischen Karriere fortzuführen. ${ }^{121,123}$

Aus diesem Grund entschied sich die Arbeitsgruppe bei der Konzipierung des Kurses, mit Peer-Dozierenden zu arbeiten. Diese wurden vor Beginn des ersten Durchganges rekrutiert und wie von Expert:innen empfohlen, speziell für die Erste-HilfeKurse geschult. ${ }^{109}$ Hierfür wurde ausgehend von den oben beschriebenen Vorarbeiten der Arbeitsgruppe ein zweitägiges Seminar für die Tutor:innen erstellt. Die Veranstaltung wurde in enger Zusammenarbeit mit den Lehrkoordinatorinnen der Chirurgie, ärztlichen Dozierenden, Unterrichtsbeauftragten sowie den erfahreneren studentischen Hilfskräften, die an der Entwicklung des Kurskonzepts beteiligt waren, durchgeführt. Im Rahmen des Qualitätsmanagements wurden die Instruktor:innen zusätzlich zu den theoretischen und praktischen Inhalten didaktisch geschult. Hier 
lag der Fokus auf den „Kenntnissen in der Motivation und Ausbildung von Erwachsenen ${ }^{124 “}$, wie Kanz et al. ${ }^{124}$ empfehlen und beinhaltete u.a. „Kompetenzen in Gruppenleitung, Rollenspielen, Feedbackgabe und Lerndidaktik" nach Empfehlung von Blohm et al. ${ }^{109}$.

Im Zuge des ständigen Qualitätsmanagements wiederholen die Peer-Dozierenden in regelmäßig stattfindenden Tutor:innen-Schulungen den theoretischen Inhalt des Kurses, werden nochmals methodisch-didaktisch geschult und evaluieren dabei regelmäßig das aktuelle Kurskonzept des Erste-Hilfe-Kurses.

\subsubsection{Gruppengröße}

Mahling et al. ${ }^{125}$ konnten zeigen, dass sich eine Gruppengröße von maximal acht Studierenden im BLS-Training als effektiv erwies. Die Studie von Cho et al. ${ }^{126}$ wies darauf hin, dass Studierende bei kleinerer Gruppengröße aktiver am Kurs teilnahmen und die Teilnehmenden insgesamt eine kleinere Gruppengröße bevorzugten. Dadurch, dass in diesem konzipierten Erste-Hilfe-Kurs 6 der 9 UE fast ausschließlich praktische Übungen beinhalten, entschied die Arbeitsgruppe, dass acht Kursteilnehmenden von zwei geschulten Peer-Instruktor:innen unterrichtet werden, um eine intensive Betreuung und effektive Vermittlung der notfallmedizinischen Maßnahmen zu gewährleisten. Um möglichst viele interessierte Vorklinik-Studierende schulen zu können, fanden immer zwei Kurse parallel statt.

\subsubsection{Einsatz des ABCDE-Schemas}

Abweichend von vielen anderen Kursen wurde sich beim Kurskonzept am ABCDESchema orientiert, da dieses Schema fester Bestandteil der notfallmedizinischen Algorithmen ist. Die vorgesehenen Erste-Hilfe-Kursinhalte wurden innerhalb der $A B C D E-L e i t s t r u k t u r$ gegliedert und mit vorklinischen Exkursen ergänzt.

Mehrere Studien konnten zeigen, dass das ABCDE-Schema zur strukturierten und erfolgreichen Abarbeitung eines Notfalls geeignet ist. ${ }^{91,92}$ Die Entscheidung, auch bei der Erstversorgung der Patient:innen durch Laien das ABCDE-Schema anzuwenden und die Erste-Hilfe-Inhalte darunter zu gliedern wurde vor dem Hintergrund 
getroffen, zum einen eine sinnvolle und strukturierte Abarbeitung eines Notfalles anhand des erwähnten Schemas zu gewährleisten. Zum anderen versuchte die Arbeitsgruppe eine Lernspirale zu schaffen, da den Studierenden das ABCDESchema immer wieder im Laufe ihrer klinischen Karriere begegnen wird. Somit wird versucht, schon frühzeitig und langfristig das ABCDE-Schema in die klinische Arbeit der Studierenden an Notfallpatient:innen zu etablieren.

\subsubsection{Implementierung}

In mehreren Probeläufen wurde das Kurskonzept, die Materialien und die Unterlagen optimiert. Am 07. und 08. April 2017 fand die erste Schulung der Kursdozierenden statt. Insgesamt konnten zu Beginn der Kursimplementierung elf Studierende als Dozierenden geschult werden.

Die Instruktor:innen erhielten in der Schulung das Curriculum des Kurses sowie einen genauen zeitlichen Ablaufplan. Zusätzlich wurde eine PowerPoint-Präsentation zur Unterstützung des Kurses zur Verfügung gestellt.

\subsubsection{Kursort}

Der Kurs fand in den Räumlichkeiten des Simulationszentrums der Goethe-Universität Frankfur, kurz FInesT, unter der Leitung von Prof. Dr. Miriam Rüsseler statt. In zwei Seminarräumen wurden dort getrennt voneinander acht Studierende in Kleingruppen von je zwei Peer-Dozierenden in Erster Hilfe unterrichtet. Die Räume baten für die Gruppengröße ausreichend Platz, um praktische Maßnahmen zu üben. Zusätzlich standen für die Objective Structured Clinical Examinations (OSCE) bzw. Objective Structured Practical Examinations (OSPE) Räumlichkeiten des Simulationskrankenhauses auf der gleichen Etage zur Verfügung. 


\subsubsection{Probeläufe}

Der Erste-Hilfe-Kurs wurde in mehreren Probeläufen durch die geschulten ErsteHilfe-Dozierenden, weitere Mitarbeiter:innen des FIneST sowie durch ärztliches Personal getestet und basierend auf den Evaluationen weiter überarbeitet.

\subsubsection{Kursbeginn}

Die Kurse wurden im Mai und Juni 2017 jeden Samstag durchgeführt. Die Studierenden hatten die Möglichkeit, sich für die vorgegebenen fünf Termine vorab anzumelden. Aufgrund der hohen Nachfrage und um alle interessierten Studierenden in Erster Hilfe schulen zu können, wurden im Sommersemester 2017 jeweils drei Zusatztermine unter der Woche ermöglicht. Der zweite Durchlauf der Erste-Hilfe-Kurse fand im November und Dezember 2017 statt.

\subsubsection{Evaluation}

Durch die kontinuierliche Qualitätskontrolle im Rahmen einer regelmäßigen Evaluation der Studierenden konnte die Qualität der Unterrichtsdurchführung gesteigert werden. ${ }^{124}$ Aus diesem Grund fand im Rahmen des Qualitätsmanagements nach jedem Kurs die schriftlichen Evaluationen durch die Teilnehmenden statt.

\begin{tabular}{|l|l|}
\hline 1. & Allgemeines zum Kurs \\
Wie bewertet Ihr den Kurs insgesamt? \\
Wie war die Atmosphäre im Kurs? \\
Wie war das Auftreten/die Kommunikation des Instruktors? \\
\hline Wie habt ihr Euch in unseren Räumlichkeiten gefühlt? \\
\hline 2. & Struktur und Aufbau des Kurses \\
\hline Die Dauer des Kurses war insgesamt \\
\hline Der Praxisanteil war \\
\hline Der Theorieanteil war \\
\hline Die Pausen waren \\
\hline 3. & Das Erste-Hilfe Training war \\
\hline
\end{tabular}




\begin{tabular}{|l|l|}
\hline & Theoretisch interessant \\
\hline Notwendig \\
\hline Praktisch für mich persönlich umsetzbar \\
\hline 4. & Deinen Vorstellungen entsprechend \\
\hline Hat klar und deutlich gesprochen \\
\hline Geht angemessen auf Anregungen und Fragen ein \\
\hline Erklärt Sachverhalte und Zusammenhänge verständlich \\
\hline Vermittelt den Stoff in einem angenehmen Tempo \\
\hline 5. & Räumlichkeiten und Material \\
\hline Der Kursraum war groß genug \\
\hline Die Anzahl der Teilnehmenden war angemessen \\
\hline Es gab ausreichend Material zum Üben \\
\hline 6. & $\begin{array}{l}\text { Würdest Du den Kurs weiterempfehlen und wenn ja warum, bzw. warum } \\
\text { nicht? }\end{array}$ \\
\hline 7. & Hilft dir das vorgestellte ABCDE-Schema im Notfall? \\
\hline 8. & Ich fühle mich nach dem Kurs in der Lage, professionell Erste-Hilfe zu \\
leisten?
\end{tabular}

Tabelle 7: Fragen der Evaluation am Ende des Erste-Hilfe-Kurses.

Die Ergebnisse der studentischen Evaluationen wurden nach Durchlauf eines KursBlockes in der Arbeitsgruppe anonymisiert vorgestellt und auf dieser Grundlage der Kurs optimiert. Ebenfalls Bestandteil der Vorstellung war, wie von Kromrey et al. ${ }^{127}$ empfohlen, der mündliche Evaluations-Bericht der Peer-Dozierenden.

\subsection{Studienvorbereitung}

Basierend auf den Fragestellungen und Hypothesen der vorliegenden Arbeit wurde sich für ein Prä-Post-Studiendesign entschieden. Es zeigte sich, dass eine möglichst objektive Abfrage der Kompetenzen bei Medizinstudierenden die Verwendung von 
unterschiedlichen Instrumenten erfordert. ${ }^{128}$ Aus diesem Grund wurde ein Selbsteinschätzungsbogen, zehn Multiple-Choice-Fragen (MC-Fragen) zur theoretischen Wissensabfrage sowie drei OSCE-/OSPE-Stationen ausgewählt.

\subsubsection{Selbsteinschätzungsbogen}

Für die Bewertung des Wissens- und Kompetenzerwerbs der Studierenden wurde zunächst ein Selbsteinschätzungsbogen (Tab. 8) erarbeitet.

1. Wenn jemand einen Herzstillstand in deiner Gegenwart erleidet, würdest du Wiederbelebungsmaßnahmen durchführen?

2. Fühlst du dich momentan in der Lage Wiederbelebungsmaßnahmen durchzuführen?

3. Wie sicher fühlst du dich in den einzelnen Maßnahmen?

Ansprechen, Hilfe rufen, Atmung überprüfen, Notruf absetzen, Herzdruckmassage, Anwendung eines AED, Beatmung

4. Wie oft sollte ein solcher Kurs deiner Meinung nach wiederholt werden?

5. Erachtest du es als sinnvoll, einen Erste-Hilfe-Kurs als Zulassungsvoraussetzung für das Physikum absolvieren zu müssen?

Tabelle 8: Fragen des Selbsteinschätzungsbogens zum Messzeitpunkt 1 und 2.

Ein besonderer Fokus lag dabei auf der Einschätzung der Sicherheit in elementaren Maßnahmen innerhalb der HLW mit einer 3-Punkte-Likert-Skala („Sehr sicher“, „Sicher", „Unsicher"). Des Weiteren wurde erfragt, wann die Studierenden die Wiederholung eines Erste-Hilfe-Kurses ansetzen würden („alle 6 Monate“, „einmal im Jahr“, „alle 2 Jahre“, „alle 5 Jahre“, „alle 10 Jahre“, „gar nicht") und ob sie es als sinnvoll erachten, den Kurs als Zulassungsvoraussetzung für die Anmeldung zum Ersten Abschnitt der Ärztlichen Prüfung zu absolvieren. 


\subsubsection{Multiple-Choice-Fragen zur Wissensüberprüfung}

Neben der Selbsteinschätzung wurde das Faktenwissen mittels zehn MC-Fragen erfasst. Bereits seit 1970 werden in Deutschland MC-Fragen in den zentral organisierten Ärztlichen Prüfungen eingesetzt. ${ }^{129}$ Aufgrund vorherrschender guter Erfahrungen mit dieser Fragenform zur Überprüfung des theoretischen Kenntnisstandes der Studierenden, entschied sich die Arbeitsgruppe dafür. Dabei wurden formale Gütekriterien zur Steigerung von Validität, Reliabilität und Objektivität der MC-Fragen beachtet. ${ }^{130}$ Nach Empfehlung von Öchsner et al. ${ }^{131}$ wurden vorab hochwertig konstruierte und methodisch vorbildliche MC-Fragen zum Thema Erste Hilfe gesichtet und daraus zehn Fragen abgeleitet (Tab. 9).

Die Studierenden beantworteten die Fragen online auf Tablets. Die Dauer der Bearbeitung war dabei nicht festgeschrieben, es wurden keine Hilfsmittel zugelassen. Die Fragen wurden aus dem Kontext der Ersten Hilfe ausgewählt.
1. Du siehst jemanden kollabieren, wie gehst du als erstes vor?
2. Wie lange versuchst du herauszufinden, ob die Person einen Kreislauf hat?
3. Wie schnell drückt man bei der Herzdruckmassage?
4. Wie viele Herzdruckmassagen führst du im Rahmen eines Zyklus beim Herz- kreislaufstillstand durch?
5. Welches der folgenden Zeichen ist KEIN sicheres Zeichen für eine Fraktur?
6. Was gehört zu den Anzeichen eines Schlaganfalls?
7. Im Supermarkt triffst du auf einen Kunden, der über starke Brustschmerzen klagt. Wie gehst du weiter mit inm vor?
8. Du bist Zeuge eines Verkehrsunfalls. Ein Motorradfahrer liegt bewusstlos auf der Straße. Du nimmst den Helm...
9. Du versorgst eine stark blutende Wunde, wie gehst du dabei als erstes vor?
10. Du sollst einen Notruf absetzen, welche Nummer wählst du?

Tabelle 9: Multiple-Choice-Fragen zum Messpunkt 1 und 2. 


\subsubsection{OSCE-/OSPE-Stationen}

Es werden bereits flächendeckend OSCE/OSPEs als Prüfungsmethode in Deutschland eingesetzt. ${ }^{132,133}$ Diese bieten nach Jünger et al. ${ }^{134}$ die „Möglichkeit einer frühzeitigen gezielten Intervention zur Förderung einer korrekten Einschätzung der eigenen Kompetenz. ${ }^{134 " ~ Z u s a ̈ t z l i c h ~ s i n d ~ d i e ~ " S e l b s t e i n s c h a ̈ t z u n g e n ~ d e r ~ a ̈ r z t l i c h e n ~}$ Kompetenzerwartung in Verbindung mit der objektiven Leistungsfeststellung mittels

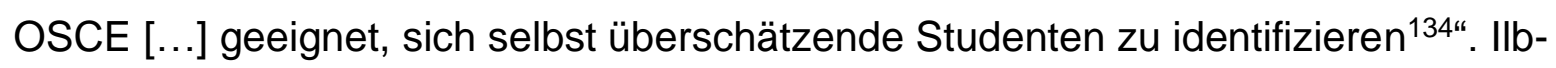
her et al. ${ }^{135}$ zeigten in ihrer Untersuchung, „dass bei Lernziel- und Blueprint-basierter Aufgabengestaltung und Einhaltung eines strukturierten Reviewprozesses befriedigende Ergebnisse hinsichtlich methodischer Kriterien auch mit relativ wenigen [notfallmedizinischen OSCE-] Stationen erreicht werden können. ${ }^{135 ،}$

Aus diesem Grund entschied sich die Arbeitsgruppe für drei OSCE-/OSPE-Stationen zur Überprüfung der praktischen Kompetenzen. Die praktischen Stationen orientieren sich an den zu vermittelnden Erste-Hilfe-Kursinhalten und überprüfen einfache Erstmaßnahmen im Rahmen eines Notfalls.

\section{Reanimation bei Herz-Kreislauf-Stillstand}

2. Seitenlage bei Bewusstlosigkeit

3. Reanimation und Anwendung eines AED bei Herz-Kreislauf-Stillstand

Tabelle 10: Die drei OSCE/OSPE-Stationen zum Messzeitpunkt 1 und 2.

Um die CPR-Maßnahmen der Studierenden bewerten zu können, wurden Simulationspuppen mit einem virtuellen Feedback-System genutzt. Über eine Anzeige auf einem SimPad, welches mit der Puppe verbunden ist, erhalten die Prüfenden eine Rückmeldung über die CPR-Qualität des Geprüften. Es werden die Drucktiefe, Frequenz und korrekte Entlastung angezeigt und farblich mit grün (korrekte Durchführung) oder rot (nicht korrekte Durchführung) verdeutlicht. 


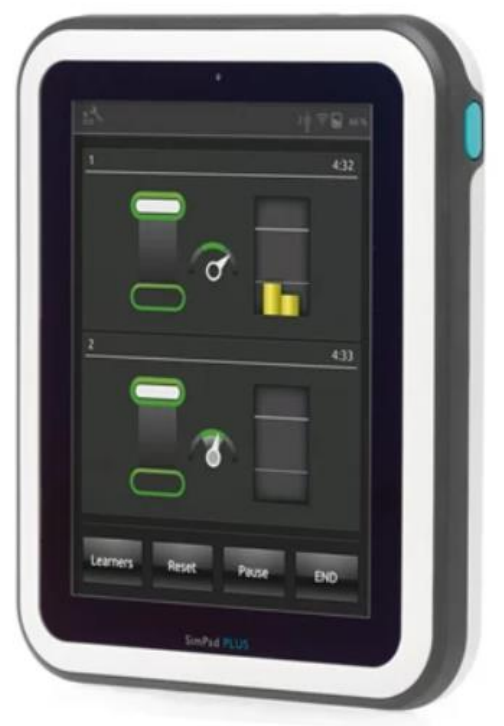

Abbildung 6: SimPad SkillReporter von Laerdal (@ Laerdal Medical)

\subsubsection{Blueprint}

Die Grundlage für die OSCE/OSPE-Stationen bildeten die gängigen Inhalte der Erste-Hilfe-Kurse. Hier wurde sich zunächst für die kardiopulmonale Reanimation und die stabile Seitenlage als Inhalt jeweils einer OSCE/OSPE-Station entschieden. Nach Durchlauf des ersten Kurses kam die Station „Reanimation mit AED“ hinzu, da viele Studierenden im ersten Durchlauf des Kurses bei der Nutzung des AED Unsicherheit äußerten. Basierend auf den Themen der Stationen wurden die durchzuführenden Maßnahmen sowohl definiert als auch anhand ihrer Schwere und Dringlichkeit priorisiert. Anhand der Stationen wurde ein „Blueprint” erstellt (Tab. 11), um die zu prüfenden Inhalte der Stationen mit dem zugrunde liegenden Curriculum des Kurses und den gängigen Erste-Hilfe-Kursinhalten abzugleichen.

\begin{tabular}{|l|l|l|}
\hline \multicolumn{1}{|c|}{ Problemfeld } & \multicolumn{1}{|c|}{ Szenario } & \multicolumn{1}{c|}{ Maßnahmen } \\
\hline Kreislaufstillstand & BLS & Ein-Helfer-CPR \\
\hline Kreislaufstillstand & BLS und AED & $\begin{array}{l}\text { Zwei-Helfer-CPR, Nutzung AED, Anleitung } \\
\text { Passant }\end{array}$ \\
\hline
\end{tabular}




\begin{tabular}{|l|l|l|}
\hline Synkope & $\begin{array}{l}\text { Vasovagale } \\
\text { Synkope }\end{array}$ & $\begin{array}{l}\text { Erkennen, Primärversorgung (Seitenlage), } \\
\text { weiteres Management }\end{array}$ \\
\hline
\end{tabular}

Tabelle 11: Erster Blueprint der drei OSCE-/OSPE-Stationen.

\subsubsection{Szenarienvorbereitung}

Der erstellte Blueprint war die Basis für die OSCE/OSPE-Stationen. In diesen sollte der Schwerpunkt vor allem auf der korrekten Durchführung der einzelnen Maßnahmen liegen. Dafür wurden diese als Checkliste zusammengestellt. Korrekt durchgeführte Maßnahmen wurden mit je einem Punkt bewertet, Schlüsselmaßnahmen sowie komplexe Maßnahmen teilweise mit zwei Punkten. Um die Studierenden auf die Szenarien vorzubereiten, wurden Türtexte entwickelt, die das Auffinde-Szenario möglichst kurz, aber präzise beschreiben. Anschließend an die Beschreibung der Situation fand sich eine Aufgabenstellung für die Studierenden. Die Szenarien wurden durch Notärzt:innen, Fachärzt:innen und durch Mitarbeitende des FIneST revidiert.

Szenario „Kardiopulmonale Ein-Helfer-Reanimation“

\begin{tabular}{|l|c|}
\hline \multicolumn{1}{|c|}{ Maßnahme } & Punkte \\
\hline Eigengefährdung ausschließen & 1 \\
\hline Ansprechen & 1 \\
\hline Vorsichtiges Schütteln an der Schulter & 1 \\
\hline „Um Hilfe rufen“ & 1 \\
\hline Atemwege freimachen & 1 \\
- Kopf reklinieren & 1 \\
- Kinn vorziehen & \\
\hline Atemkontrolle & 1 \\
- Sehen, Hören, Fühlen & 1 \\
\hline - Zeitdauer max. 10 Sekunden & 2 \\
\hline Veranlassen eines Notrufs & \\
\hline
\end{tabular}




\begin{tabular}{|l|l|}
\hline Aufsuchen des korrekten Druckpunktes & 1 \\
\hline Korrekte Hand- und Körperhaltung & 1 \\
\hline 30 Herzdruckmassagen & 1 \\
- Frequenz: 100-120 Kompressionen/Min. & 2 \\
- Drucktiefe: 5-6 cm & 2 \\
- Kompression \& Entlastung gleich lang, vollständige Entlastung & 1 \\
\hline
\end{tabular}

Tabelle 12: Checkliste der OSCE/OSPE-Station „Kardiopulmonale Ein-Helfer-Reanimation“.

\begin{tabular}{|l|c|c|}
\hline Maßnahme & Prozent & Punkte \\
\hline Frequenz & $80 \%$ & 2 \\
\hline Drucktiefe & $60 \%$ & 1 \\
\hline Kompression \& Entlastung & $80 \%$ & 2 \\
\hline
\end{tabular}

Tabelle 13: Ergänzung der Checkliste zur OSCE/OSPE-Station „Kardiopulmonale Ein-Helfer-Reanimation“.

Erwartet wurde, dass die Studienproband:innen zunächst auf die Eigengefährdung achten, diese ausschließen und anschließend einen klaren Patientenkontakt durch Ansprache und vorsichtiges Schütteln herstellen. Eine weitere Möglichkeit war der Weckversuch durch einen Schmerzreiz. Die Studierenden sollten frühzeitig um Hilfe rufen und mit dem Freimachen der Atemwege beginnen. Darunter fielen die Maßnahmen „Kopf reklinieren“ und „Kinn vorziehen“. Anschließend sollte die Atemkontrolle maximal zehn Sekunden mit „Sehen, Hören und Fühlen“ der Atmung stattfinden. Spätestens nach Feststellung der fehlenden Atmung sollte der Notruf korrekt unter der Notrufnummer 112 mit Informationen zu Notfallort und Notfallsituation abgegeben werden. Dies wurde als Schlüsselmaßnahme mit zwei Punkten bewertet. Danach sollten die Proband:innen den korrekten Druckpunkt aufsuchen und mit 30 Herzdruckmassagen in korrekter Hand- und Körperhaltung beginnen. Dabei war auf die richtige Frequenz mit 100-120 Kompressionen/Minute, die Drucktiefe mit mindestens $5 \mathrm{~cm}$ und maximal $6 \mathrm{~cm}$ sowie auf eine vollständige Entlastung zu achten. 
Wenn die Teilnehmenden bei $80 \%$ der Zeit suffizient reanimierten, wurden die Frequenz und Drucktiefe als Schlüsselmaßnahmen bewertet und die Teilnehmenden erhielten jeweils zwei Punkte. Bei $60 \%$ suffizient durchgeführten Thoraxkompressionen erhielten die Teilnehmenden einen Punkt. Sobald die Teilnehmenden mit den geforderten 30 Herzdruckmassagen fertig waren und Zeit übrigblieb, wurden sie aufgefordert, weiter die Herzdruckmassage weiterzuführen.

Szenario „Vasovagale Synkope“

\begin{tabular}{|l|c|}
\hline \multicolumn{1}{|c|}{ Maßnahme } & Punkte \\
\hline Ausschließen Eigengefährdung & 1 \\
\hline Ansprechen & 1 \\
\hline Vorsichtiges Schütteln an der Schulter & 1 \\
\hline „Um Hilfe rufen“ & 1 \\
\hline Atemwege freimachen & \\
- Kopf reklinieren & 1 \\
- Kinn vorziehen & 1 \\
\hline $\begin{array}{l}\text { Atemkontrolle } \\
\text { - Sehen, Hören, Fühlen }\end{array}$ & 1 \\
- Zeitdauer max. 10 Sekunden & 1 \\
\hline $\begin{array}{l}\text { Veranlassen eines Notrufs } \\
\text { Lagerung der Patientin in Rückenlage, Position des Studierenden } \\
\text { kniend seitlich der Patientin }\end{array}$ & 2 \\
\hline $\begin{array}{l}\text { Korrekte Lagerung in eine stabile Seitenlage, ohne die Patientin zu } \\
\text { verletzen } \\
\text { Überstrecken des Kopfes und Öffnen des Mundes }\end{array}$ & 1 \\
\hline
\end{tabular}

Tabelle 14: Checkliste der OSCE/OSPE-Station „Stabile Seitenlage“.

Nach Ausschließen der Eigengefährdung sollten die Proband:innen die Patientin ansprechen und durch vorsichtiges Schütteln an der Schulter den Bewusstseinsstatus überprüfen. Auch hier sollten die Atemwege mittels Kopfreklination und Vorziehen 
des Kinns freigemacht und die Atmung mittels „Sehen, Hören, Fühlen“ maximal 10 Sekunden überprüft werden. Die Probanden sollten frühzeitig um Hilfe rufen.

Nach Ansage durch die Prüfenden, dass eine Atmung hörbar ist, sollte die Patientin in die korrekte Lagerung in Form einer Seitenlage gebracht werden, ohne sie dabei zu verletzen. Schlüsselmaßnahmen an dieser Station und mit je zwei Punkten bewertet, waren wieder das Veranlassen eines Notrufs, das Überstrecken des Kopfes und Öffnen des Mundes der Patientin am Ende der Seitenlagerung.

Szenario „Kardiopulmonale Zwei-Helfer-Reanimation mit AED“

\begin{tabular}{|l|c|}
\hline \multicolumn{1}{|c|}{ Maßnahme } & Punkte \\
\hline Eigengefährdung ausschließen & 1 \\
\hline Startet mit der CPR & 1 \\
\hline Bindet den Passanten sinnvoll bei der CPR ein & 1 \\
\hline Setzt den AED ein & 2 \\
\hline Korrekte Befolgung der Anweisungen & 1 \\
\hline Beachtet eine sinnvolle Reihenfolge (1; 2; 3; 4): & 2 \\
Anschalten ( ), Aufkleben ( ), Anschließen ( ), Analyse ( ) & \\
\hline Gibt Kommando: „Alle weg vom Patienten“ & 1 \\
\hline Kontrolle, dass keiner den Patienten berührt & 2 \\
\hline Gibt Kommando: „Achtung Schock“ & 1 \\
\hline Auslösung des Schocks & 1 \\
\hline $\begin{array}{l}\text { Startet zügig weiter mit CPR } \\
\text { verzögert (_min) }\end{array}$ & 1 \\
\hline
\end{tabular}

Tabelle 15: Checkliste der OSCE/OSPE-Station „Kardiopulmonale Zwei-Helfer-Reanimation mit AED“.

In dieser Notfallsituation fanden die Studierenden einen Schauspieler in der Rolle eines Passanten bei einer leblosen Person vor. Der Notruf wurde durch den Passanten bereits abgesetzt. Die Aufgabe der Proband:innen war, nach Ausschluss der Eigengefährdung mit der Reanimation zu beginnen und vorhandene Hilfsmittel für 
die Wiederbelebung einzusetzen. Es wurde erwartet, dass die Reanimation leitliniengerecht begonnen und nach Möglichkeit der Passant als Hilfe in die Reanimation eingebunden wird. Des Weiteren sollte der AED eingesetzt, ordnungsgemäß verwendet und gestartet werden. Die Teilnehmenden sollten den akustischen Anweisungen des AED korrekt Folge leisten und die eigene sowie die Sicherheit des Passanten bei der Schockabgabe im Blick behalten. Dafür wurden Kommandos, den Patienten nicht zu berühren sowie das Kommando der Schockabgabe mit jeweils einem Punkt bewertet. Die Kontrolle, dass keiner der Anwesenden den Patienten berührt, wurde als Schlüsselmaßnahme der sicheren Durchführung des Schockes mit je zwei Punkten bewertet.

Nach Absetzen des ersten Schocks sollte sofort die Herzdruckmassage korrekt fortgesetzt werden, um eine No-flow-time möglichst zu vermeiden. Eine eventuell stattgefundene Zeitverzögerung wurde entsprechend dokumentiert.

\subsubsection{Simulator}

In den drei OSCE/OSPE-Stationen wurden verschiedene Simulatoren eingesetzt (Tab.16). In den beiden Stationen mit CPR wurden Rescuci Anne Simulatoren (Laerdal, Stavanger, Norwegen. Copyright (c) 2020 Laerdal Medical) genutzt. Diese funktionieren mittels WLAN und sind dadurch ideal durch die Prüfenden steuerbar. Durch Knopfdruck können Herz-, Atemfrequenz und Blutdruck modifiziert werden, sodass zum Beispiel der Trainings-AED den eingestellten Rhythmus erkennt und automatisch in den defibrillierbaren Modus springt.

Des Weiteren lassen sich mittels des Touchpads die genaue Drucktiefe, Frequenz und Entlastung bei der Reanimation ablesen, was sich für die objektive Beurteilung bei den Checklisten als essenziell erweist.

Für die Station der stabilen Seitenlage wurde ein Blueman genutzt. Vorteil dieser Puppe ist die realitätsnahe Darstellung des Körpergewichtes einer bewusstlosen Person, weswegen sich in dieser Station für diesen Simulator entschieden wurde. Da dieser nicht in der Lage ist, wie die Rescuci Anne-Simulatoren Herz-, Atemfrequenz und Blutdruck zu simulieren, wurde sich im Rahmen der vorliegenden Studie 
daher entschieden, die Prüfenden ansagen zu lassen, dass die Person atmet, wenn die Geprüften dies kontrollierten.

\begin{tabular}{|c|c|}
\hline Station & Simulator \\
\hline CPR & Anne \\
\hline Stabile Seitenlage & Blueman \\
\hline CPR mit AED & Anne \\
\hline
\end{tabular}

Tabelle 16: Einsatz von verschiedenen Simulatoren an den drei OSCE-/OSPE-Stationen.

\subsubsection{Prüfende}

Schwill et al. ${ }^{136}$ zeigten, dass auch Peer-Dozierende als Prüfende eingesetzt werden können. Aus Ressourcengründen wurde sich deshalb bei den Prüfenden der OSCE/OSPE-Stationen für Peers in Form von erfahrenen BLS-Instruktor:innen des FIneST entschieden. Sie erhielten vorab eine Einführung in den Ablauf sowie die Prüfungsbögen und prüften sowohl den ersten Durchlauf vor dem Erste-Hilfe-Kurs als auch den zweiten Durchlauf danach.

\subsubsection{Durchführbarkeitsanalyse der OSCE/OSPE-Stationen}

Alle geplanten OSCE/OSPE-Stationen wurden im Vorfeld von Mitarbeiter:innen des FIneST durchlaufen. Hier sollte die Durchführbarkeit der drei Stationen, die zeitliche Determinierung mit Wechselzeiten sowie die erstellten Checklisten getestet werden. Mit einer Stationsdauer von fünf Minuten konnten alle Teilnehmenden jede Station erfolgreich abschließen. Im Durchschnitt waren die Freiwilligen sogar bereits nach drei bis vier Minuten mit der Station fertig, weswegen der zeitlich gesetzte Rahmen für Laien angemessen erschien und die zeitliche Determinierung auf 5:00 Minuten für alle Stationen festgesetzt wurde.

Insgesamt wurden alle Stationen noch einmal in einem Gesamtdurchlauf mit Wechselzeiten getestet. In dieser sind die Prüfenden mit der Nachbereitung des Szenarios (Vervollständigung der Checkliste, Wiederherstellung des Auffinde-Szenarios) beschäftigt, während die Teilnehmenden die Zeit vom Beenden eines Szenarios bis 
zum Beginn der nächsten Station benötigen, um den Raum zu wechseln und den Informationstext mit der Aufgabenstellung für die nächste Station zu lesen. Insgesamt dauerte ein Durchlauf der drei Stationen 15 Minuten zuzüglich drei Minuten Wechselzeit. Beim zweiten Durchlauf der Stationen zeigte sich, dass die Teilnehmenden deutlich weniger Zeit für die Stationen und zum Wechsel benötigen, da die Stationen und die Aufgabenstellungen nun bekannt waren und die Teilnehmenden die vorausgesetzten praktischen Inhalte beherrschten. Doch um sowohl eine Vergleichbarkeit als auch einen reibungslosen Ablauf zu gewährleisten und um den Prüfenden die benötigte Zeit der objektiven Beurteilung zu geben, wurde der zeitliche Rahmen der Stationen belassen.

Im Rahmen der Testläufe konnte die Durchführbarkeit des kompletten OSCE/OSPEZyklus nachgewiesen und die zeitliche Determinierung von fünf Minuten pro Station und eine Minute Wechselzeit als sinnvoll und machbar bestätigt werden. Die Checklistenbeurteilung zeigte sich in diesem zeitlichen Rahmen ebenfalls als machbar. Die Checklisten für die drei zu absolvierenden Stationen wurden basierend auf standardisierten Algorithmen aus der Notfallmedizin erarbeitet. Im Rahmen der Testläufe konnten die Checklisten basierend auf dem Feedback der Mitarbeitenden weiter optimiert werden.

\subsection{Studie}

\subsubsection{Studiendesign}

Die Studie basiert wie bereits beschrieben auf drei OSCE/OSPE-Stationen sowie zehn MC-Fragen und einer Selbsteinschätzung vor Durchführung des Kurses, sowie auf denselben drei OSCE/OSPE-Stationen und MC-Fragen sowie einer Kursevaluation danach.

Die Studierenden wurden von erfahrenen Erste-Hilfe-Instruktor:innen des FIneST geprüft. Die Prüfenden erhielten vor Beginn der Studie eine Einweisung in diese sowie ein standardisiertes Training zur Durchführung der OSCE/OSPE-Stationen mittels der Checklistenbeurteilung. 


\subsubsection{Studienteilnehmende}

Die Studienteilnehmenden waren Studierende des Studiengangs Humanmedizin im vorklinischen Studienabschnitt an der Goethe-Universität Frankfurt am Main, die den Erste-Hilfe-Kurs absolvieren wollten. Die Studierenden wurden vor Beginn des Kurses über die geplante Studie informiert. Bei Interesse erfolgte die ausführliche Aufklärung über die Studie. Die Studieneinwilligung erfolgte schriftlich, jeder Studienteilnehmende erhielt zudem eine Kopie der Aufklärung. Die Teilnahme an der Studie war für die Studierenden freiwillig und erfolgte nach schriftlicher Einverständniserklärung, die jederzeit widerrufbar war.

\subsubsection{Studienprotokoll}

Alle Teilnehmenden bekamen zu Beginn der OSCE/OSPE-Prüfung eine kurze Einführung in den generellen Ablauf, auf welches Kommando sie die Stationen betreten sollen, die Zeit, die sie innerhalb der Stationen haben, sowie die Wechselzeit zwischen den Stationen. Alle Studienteilnehmenden absolvieren den oben beschrieben regulären Erste-Hilfe-Kurs. Vor Kursbeginn starten sie jedoch im Vergleich zu den Teilnehmenden ohne Studienteilnahme mit dem Messzeitpunkt 1 (Abb. 7).

\begin{tabular}{|l|l|}
\hline Messzeitpunkt 1 & $\begin{array}{l}\text { 3 OSCE/OSCPE-Stationen } \\
\text { Fragebogen Selbsteinschätzung } \\
\text { 10 MC Fragen }\end{array}$ \\
Erste Hilfe Kurs & \\
Messzeitpunkt 2 & $\begin{array}{l}\text { 3 OSCE/OSCPE-Stationen } \\
\text { Fragebogen Selbsteinschätzung } \\
\text { 10 MC Fragen }\end{array}$ \\
\hline
\end{tabular}

Abbildung 7: Schematische Darstellung des Studienprotokolls. 


\subsubsection{Messzeitpunkt 1}

Am Messzeitpunkt 1 erhielten die Studienteilnehmenden vor Beginn des Durchlaufs eine 5-minütige Einführung in den Ablauf der OSCE/OSPE-Stationen. In dieser wurde auf den Ablauf der Rotation, die Wechselzeit zwischen den Stationen sowie die Funktion der Prüferinnen und Prüfer eingegangen. Anschließend wurde der Durchlauf der drei OSCE/OSPE-Stationen gestartet. Insgesamt benötigte jeder Studierende 18 Minuten, um die praktischen Stationen zu bewältigen (5 Minuten pro Station, 1 Minute Wechselzeit). Durch ein Tonsignal wurden die Wechsel zwischen den einzelnen Stationen angekündigt. Am Ende des praktischen Teils bekamen die Studierenden Touchpads ausgehändigt, auf denen sie online den Eingangsfragebogen beantworteten. Dieser enthielt außer der Abfrage demografischer Daten eine

Selbsteinschätzung sowie die zehn MC-Fragen zu deb Erste-Hilfe-Maßnahmen. Nach Beendigung der Stationen schloss sich der aus 9 UE bestehende Erste-HilfeKurs für Medizinstudierende an.

\subsubsection{Messzeitpunkt 2}

Im Anschluss an den Erste-Hilfe-Kurs durchliefen die Teilnehmenden erneut die OSCE/OSPE-Stationen und beantworteten dieselben MC-Fragen wie zuvor, sowie den Selbsteinschätzungsbogen. Zusätzlich füllten alle Teilnehmenden einen Evaluationsbogen aus. An jedem Durchlauf nahmen maximal 16 Studierende teil.

\subsubsection{Datenauswertung}

Die Ergebnisse der OSCE/OSPE-Stationen wurden in Bewertungsbögen festgehalten und anschließend ausgewertet. Die Ergebnisse der Multiple-Choice-Fragen wurden online gesammelt. Anschließend erfolgte die Eingabe der Ergebnisse der Checklisten und der Evaluationen in Microsoft Excel (Microsoft Office Excel 2007, (C) Microsoft Corporation). Die Auswertung der arithmetischen Mittelwerte, der Standardabweichung und der Evaluationen erfolgte ebenfalls in Excel, die Datenauswertung mittels SPSS (@ IBM,Armonk, USA). 
Bei der subjektiven Einschätzung und den MC-Fragen wurden zur deskriptiven Übersicht Häufigkeitsverteilungen sowie deskriptive Statistiken (arithmetischer Mittelwert, Standardabweichung, Median, Minimum, Maximum) ausgerechnet. Die dichotom (0/1) kodierten Einzelitems wurden mit Hilfe des nichtparametrischen $\mathrm{McNe}$ mar-Tests auf signifikante Unterschiede zwischen den beiden Messzeitpunkten getestet und die Unterschiede anhand deskriptiver Verteilungen in Kreuztabellen dargestellt. Bei den ordinal skalierten Selbsteinschätzungen erfolgte die Testung auf signifikante Unterschiede zwischen Messzeitpunkt 1 und Messzeitpunkt 2 mittels des nichtparametrischen Wilcoxon-Tests.

Bei den MC-Fragen wurden bivariate deskriptive Verteilungen in Kreuztabellen ausgegeben und anhand des Chi-Quadrat-Tests geprüft, ob bei den analysierten Daten und deren Verteilung ein statistisch signifikanter Zusammenhang vorliegt oder nicht. Das verwendete Signifikanzniveau war $p<0,05$.

In der Kursevaluation wurden mehrere Kriterien abgefragt, zunächst in einer 5Punkte-Likert-Skala (,sehr gut“/,gut“/,,mittelmäßig“/,schlecht“/,sehr schlecht") Allgemeines zum Erste-Hilfe-Kurs, dann mit einer 5-Punkte-Likert-Skala („zu lang“/,länger als geplant“/,,optimal“/,kürzer als geplant“/,zu kurz“) die generelle Struktur und der Aufbau des Kurses. Der Inhalt des Erste-Hilfe-Trainings, die Qualität der Instruktor:innen sowie die Räumlichkeiten und das Material wurden noch einmal gesondert mit einer 5-Punkte-Likert-Skala („trifft zu“/,trifft eher zu“/,teils-teils“/,trifft eher nicht zu“/,trifft nicht zu“) abgefragt.

Bei den praktischen Stationen wurden zur deskriptiven Übersicht Häufigkeitsverteilungen sowie deskriptive Statistiken (arithmetischer Mittelwert, Standardabweichungen, Minimum, Maximum) angegeben. Zur Testung der nicht normalverteilten Punkte und Prozente und der ordinal skalierten Einzelitems auf signifikante Unterschiede zwischen Messzeitpunkt 1 und Messzeitpunkt 2 wurde der nichtparametrische Wilcoxon-Test verwendet. Zur Testung der dichotom (0/1) kodierten Einzelitems auf signifikante Unterschiede zwischen den beiden Zeitpunkten wurde der nichtparametrische McNemar-Test verwendet. 
Um die jeweiligen Unterschiede darzustellen, wurden deskriptive bivariate Verteilungen in Kreuztabellen ausgegeben. Das verwendete Signifikanzniveau war $p<0,05$. An den beiden OSCE/OSPE-Stationen, die eine Reanimationssituation beinhalteten, konnten bei den beiden Einzelitems „Frequenz" und „Drucktiefe“ 0,1 oder 2 Punkte erreicht werden. Somit lag eine ordinale Skalierung vor, die anschließend mit dem Wilcoxon-Test getestet wurden. Des Weiteren wurden der arithmetische Mittelwert, der Median sowie die Standardabweichung zu den Items „Frequenz“ und „Drucktiefe“ berechnet. 


\section{Ergebnisse}

\subsection{Allgemein}

Insgesamt haben 232 Studierende im Studienzeitraum vom Sommersemester 2017 bis zum Sommersemester 2018 den Erste-Hilfe-Kurs absolviert. Hiervon haben 160 Studierende (69\%) an der Studienteilnahme eingewilligt. Tabelle 17 zeigt die Anzahl der Studienteilnehmenden, die an den einzelnen Studienkomponenten teilgenommen haben.

\begin{tabular}{|l|c|c|}
\hline & Messzeitpunkt 1 & Messzeitpunkt 2 \\
\hline Demographische Daten & 152 & entfällt \\
\hline Selbsteinschätzung & 152 & 152 \\
\hline MC-Fragen & 152 & 152 \\
\hline OSCE/OSPE 1 - Reanimation & 159 & 159 \\
\hline OSCE/OSPE 2 - Stabile Seitenlage & 160 & 160 \\
\hline OSCE/OSPE 3 - Einsatz AED & 91 & 91 \\
\hline
\end{tabular}

Tabelle 17: Anzahl der Studienteilnehmenden an den Messzeitpunkten vor dem Kurs (1) und danach (2).

\subsection{Demographische Daten}

Es konnten von insgesamt 152 Teilnehmenden die demographischen Daten erhoben werden. Der größte Anteil der Studienteilnehmenden mit 65,1\% war im 4. klinischen Semester.

6,3\% der Studierenden gab an, vor Studiumsbeginn eine Berufsausbildung absolviert zu haben. Genannt wurden hier die Ausbildung zur Physiotherapeut:in, Biologielaborant:in und ein Teilnehmer hatte die Ausbildung zum Rettungssanitäter abgeschlossen. Des Weiteren wurden als bereits abgeschlossene Studiengänge Bachelor of Science, Biowissenschaften und Zahnmedizin genannt.

Insgesamt haben $76,3 \%$ der Teilnehmenden vor Kursteilnahme an mindestens einem Erste-Hilfe-Kurs teilgenommen, welcher meist mehr als 3 Jahre zurück lag 
(32,5\%). 13,2\% der Studierenden haben bereits ein Reanimationstraining durchlaufen. Auch hier lag das Training bei den meisten mehr als 3 Jahre zurück (50,0\%). Nur $12,5 \%$ aller teilnehmenden Vorklinikstudierenden gab an, schon einmal selbst Erste Hilfe geleistet zu haben.

\begin{tabular}{|l|c|}
\hline & $\mathbf{n} \%$ \\
\hline Semester & $0,6 \%$ \\
\hline - 1. Klinisches Semester & $17,1 \%$ \\
- 2. Klinisches Semester & $0,9 \%$ \\
- 3. Klinisches Semester & $65,1 \%$ \\
\hline - 4. Klinisches Semester & 0 \\
- 5. Klinisches Semester & $0,6 \%$ \\
- 6. Klinisches Semester & $6,6 \%$ \\
\hline - keine Angabe & $6,3 \%$ \\
\hline Abgeschlossene Berufsausbildung & $76,3 \%$ \\
\hline Vorherige Teilnahme an Erste-Hilfe-Kurs & \\
\hline Zeitpunkt des letzten Kurses & $0,8 \%$ \\
- bis vor 6 Monaten & $1,7 \%$ \\
\hline - 7-12 Monate & $15,3 \%$ \\
- vor 2 Jahre & $29,7 \%$ \\
\hline - vor 3 Jahren & $52,5 \%$ \\
\hline - vor mehr als 3 Jahren & $13,2 \%$ \\
\hline Teilnahme an einem Reanimationstraining & \\
\hline Zeitpunkt des letzten Trainings & $5,0 \%$ \\
\hline - bis vor 6 Monaten & $15,0 \%$ \\
\hline - 7-12 Monate & $10,0 \%$ \\
\hline - vor 2 Jahre & $20,0 \%$ \\
\hline - vor 3 Jahren & $50,0 \%$ \\
\hline - vor mehr als 3 Jahren & $12,5 \%$ \\
\hline Schon einmal selbst Erste Hilfe geleistet & \\
\hline & \\
\hline
\end{tabular}


Tabelle 18: Demographische Daten der Teilnehmenden, dargestellt als prozentualer Anteil.

Tabelle 19 zeigt die von den Teilnehmenden angegebenen Gründe zur Teilnahme am Kurs. Über $80 \%$ der Studierenden nahmen am Kurs teil, um eine Kursbescheinigung zur Vorlage für die Anmeldung zum 1. Abschnitt der Ärztlichen Prüfung zu erwerben. Lediglich 5,3\% gaben an, aus persönlichem Interesse teilzunehmen.

\begin{tabular}{|l|c|}
\hline \multicolumn{2}{|l|}{ Subjektive Gründe für die Teilnahme am Kurs } \\
\hline Kurs wird für das Physikum benötigt & $82,9 \%$ \\
\hline Interesse an den Inhalten des Kurses & $5,3 \%$ \\
\hline Andere Gründe & $1,3 \%$ \\
\hline Keine Angabe & $9,9 \%$ \\
\hline
\end{tabular}

Tabelle 19: Gründe der Teilnehmenden für die Teilnahme am Kurs, dargestellt als prozentualer Anteil.

\subsection{Ergebnisse der Selbsteinschätzung}

Die teilnehmenden Studierenden schätzten sich vor dem Erste-Hilfe-Kurs durchweg unsicher in den Einzelmaßnahmen der HLW ein. Nach dem Kurs zeigte sich in allen Punkten nach dem Erste-Hilfe-Kurs eine signifikante Steigerung der Selbsteinschätzung bei „Sehr sicher“. Zur Testung auf signifikante Unterschiede zwischen Messzeitpunkt 1 und Messzeitpunkt 2 wurde der Wilcoxon-Test verwendet. Bei der Selbsteinschätzung zu den Maßnahmen besteht zwischen den beiden Zeitpunkten jeweils ein hochsignifikanter Unterschied ( $p$-Wert jeweils $<0,001$ ). In den Basismaßnahmen „Ansprechen“, „Hilfe rufen“, „Notruf absetzen“ und „Herzdruckmassage“ fühlte sich nach der Kursteilnahme keiner der Studierenden unsicher. Tabelle 20 zeigt die abgefragten Maßnahmen sowie die Selbsteinschätzung der Studierenden.

\begin{tabular}{|l|l|c|c|c|}
\hline Maßnahmen & Einschätzung & Messzeitpunkt 1 & Messzeitpunkt 2 & p-Wert \\
& Sehr sicher & $51,4 \%$ & $82,2 \%$ & \\
\multirow{3}{*}{ Ansprechen } & Sicher & $29,0 \%$ & $17,8 \%$ & $<0,001$ \\
\cline { 2 - 5 } & Unsicher & $19,6 \%$ & 0 & \\
\hline
\end{tabular}




\begin{tabular}{|c|c|c|c|c|}
\hline \multirow{3}{*}{ Hilfe rufen } & Sehr sicher & $69,2 \%$ & $95,3 \%$ & \multirow{3}{*}{$<0,001$} \\
\hline & Sicher & $29,0 \%$ & $4,7 \%$ & \\
\hline & Unsicher & $1,9 \%$ & 0 & \\
\hline \multirow{3}{*}{$\begin{array}{l}\text { Atmung } \\
\text { überprüfen }\end{array}$} & Sehr sicher & $21,5 \%$ & $83,2 \%$ & \multirow{3}{*}{$<0,001$} \\
\hline & Sicher & $55,1 \%$ & $15,9 \%$ & \\
\hline & Unsicher & $23,4 \%$ & $0,9 \%$ & \\
\hline \multirow{3}{*}{$\begin{array}{l}\text { Notruf abset- } \\
\text { zen }\end{array}$} & Sehr sicher & $57,0 \%$ & $93,5 \%$ & \multirow{3}{*}{$<0,001$} \\
\hline & Sicher & $37,4 \%$ & $6,5 \%$ & \\
\hline & Unsicher & $5,6 \%$ & 0 & \\
\hline \multirow{3}{*}{$\begin{array}{l}\text { Herzdruck- } \\
\text { massage }\end{array}$} & Sehr sicher & $7,5 \%$ & $60,7 \%$ & \multirow{3}{*}{$<0,001$} \\
\hline & Sicher & $24,3 \%$ & $39,3 \%$ & \\
\hline & Unsicher & $68,2 \%$ & 0 & \\
\hline \multirow{3}{*}{$\begin{array}{l}\text { Anwendung } \\
\text { AED }\end{array}$} & Sehr sicher & $0,9 \%$ & $53,3 \%$ & \multirow{3}{*}{$<0,001$} \\
\hline & Sicher & $5,6 \%$ & $44,9 \%$ & \\
\hline & Unsicher & $93,5 \%$ & $1,9 \%$ & \\
\hline \multirow{3}{*}{ Beatmung } & Sehr sicher & $1,9 \%$ & $39,3 \%$ & \multirow{3}{*}{$<0,001$} \\
\hline & Sicher & $16,8 \%$ & $53,3 \%$ & \\
\hline & Unsicher & $81,3 \%$ & $7,5 \%$ & \\
\hline
\end{tabular}

Tabelle 20: Subjektive Einschätzung der Teilnehmenden zu Wiederbelebungsmaßnahmen zum Messzeitpunkt 1 und 2, dargestellt als prozentualer Anteil der jeweiligen Nennung.

Die Schlüsselmaßnahmen „Atmung überprüfen“, „Herzdruckmassage“, „Anwendung eines AED“ und "Beatmung" sind in der Abbildung 8 im Einzelnen dargestellt. Hier zeigt sich eine signifikante Steigerung der Selbsteinschätzung nach Teilnahme am Kurs. Dennoch fühlen sich auch nach dem Kurs noch nicht alle Teilnehmenden sicher in der Durchführung dieser Schlüsselmaßnahmen. 


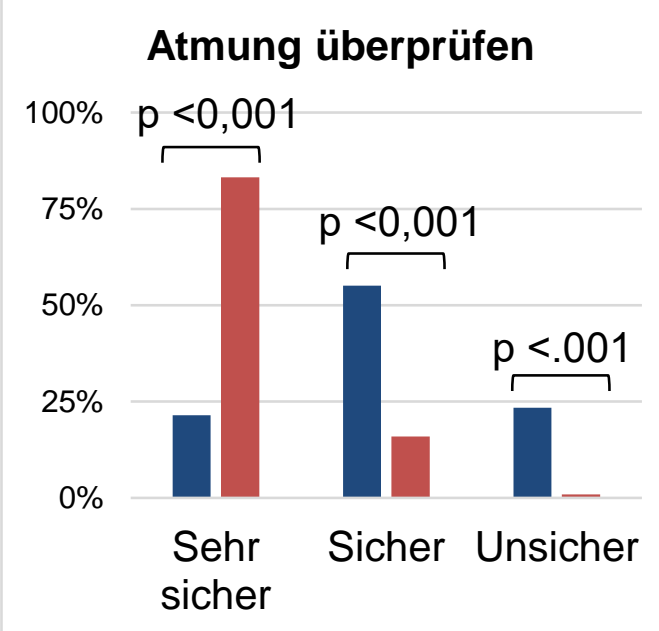

Anwendung AED

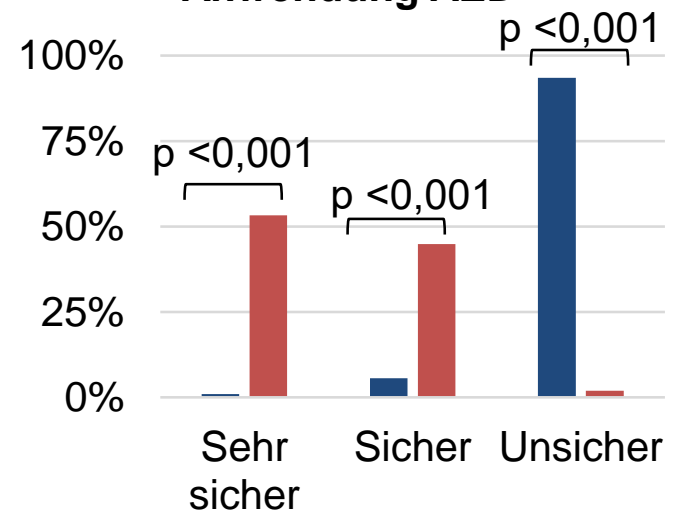

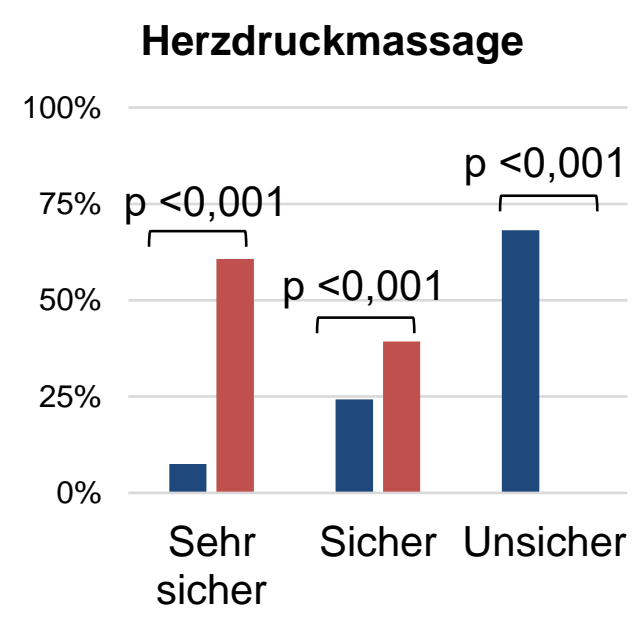

\section{Beatmung}

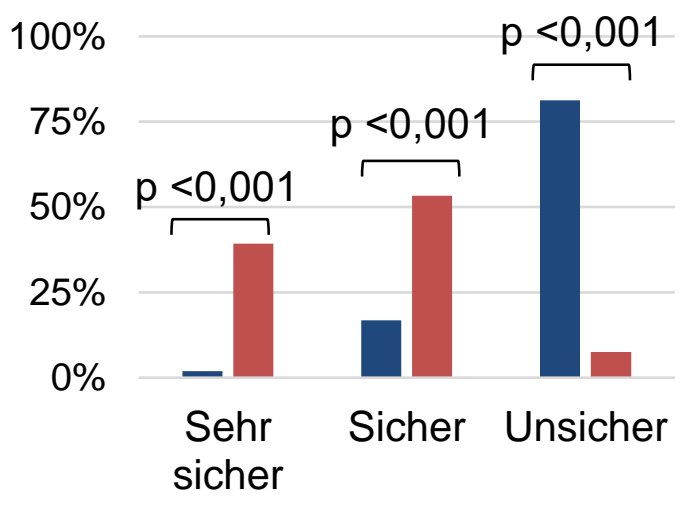

Abbildung 8: Grafische Darstellung der Schlüsselmaßnahmen der Reanimation . Messzeitpunkt 1 vor dem Kurs (blau) und Messzeitpunkt 2 nach dem Kurs (rot).

Zusätzlich zur Selbsteinschätzung wurden die Studierenden vor und nach dem Kurs gefragt, ob sie Wiederbelebungsmaßnahmen bei einem Herzstillstand durchführen würden. Nach der Kursteilnahme gaben signifikant mehr Studierende an, Wiederbelebungsmaßnahmen durchzuführen als vor dem Kurs (prä = 87,9\%; post $=98,1 \%$, $p<0,007)$. Die Teilnehmenden, die vor dem Kurs keine Wiederbelebung durchführen würden, nannten als häufigsten Grund, dass sie nicht wüssten, wie man eine HLW durchführt (66,7\%). Weitere Gründe waren die Angst, den Menschen zu verletzten, Angst vor Infektion und Angst vor rechtlichem Belangen. 
Nur 31,8\% der Studierenden fühlten sich vor dem Erste-Hilfe-Kurs in der Lage, Wiederbelebungsmaßnahmen durchzuführen. Zwischen Messzeitpunkt 1 und Messzeitpunkt 2 bestand ein hochsignifikanter Unterschied $(p<0,001)$ : nach Kursteilnahme trauten sich $98,1 \%$ der Studierenden zu, Wiederbelebungsmaßnahmen durchzuführen.

Bei der Frage, ob die Studierenden einen Erste-Hilfe-Kurs als Zulassungsvoraussetzung für das Physikum als sinnvoll erachten, bestand kein signifikanter Unterschied zwischen Messzeitpunkt 1 und Messzeitpunkt 2 ( $p<1,000$ ). 92,9\% hielten inn bereits vor Teilnahme des Kurses sinnvoll, nach dem Kurs waren es 95,7\%.

Sowohl vor als auch nach der Kursteilnahme befürwortete die Mehrheit der Studierenden eine Kurswiederholung alle zwei Jahre (prä $=59,0 \%$; post $=61,0 \%$ ). Obwohl sich diesbezüglich keine Änderungen zeigten, gibt es eine deutliche Zunahme des Wunsches nach einer Kurswiederholung jedes Jahr (prä $=28,0 \%$; post $=34,0 \%$ ) und eine Abnahme der Angabe "Gar nicht" (prä = 3,0\%; post = 1,0\%) und "nur alle 5 Jahre" (prä $=5,0 \%$; post $=0,0 \%$ ).

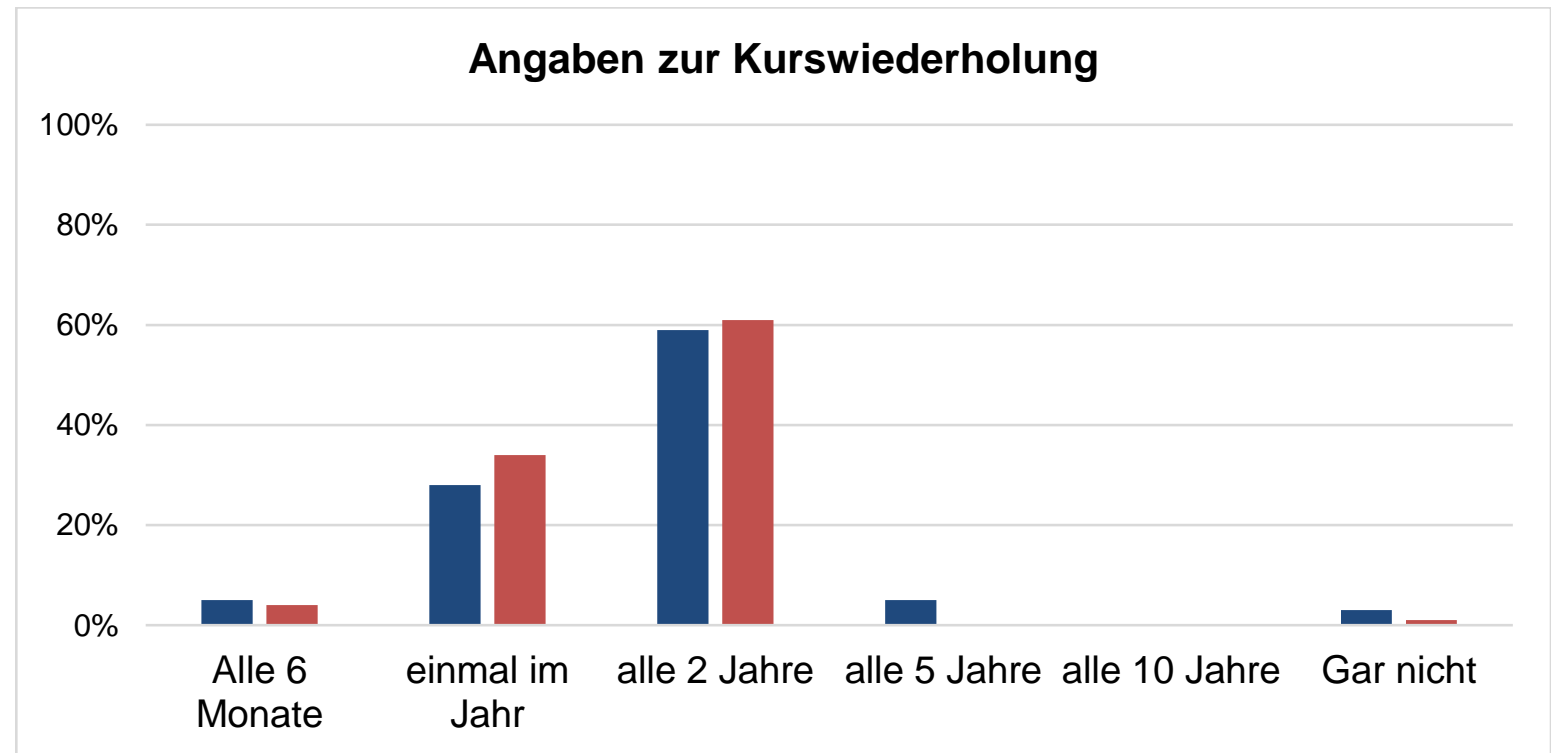

Abbildung 9: Angaben zur Kurswiederholung zum Messzeitpunkt 1 (blau) vor dem Kurs und Messzeitpunkt 2 (rot) nach dem Kurs, dargestellt als prozentualer Anteil. 


\subsection{Ergebnisse der Wissensüberprüfung}

Das theoretische Wissen der Studierenden wurde anhand eines 10-Items MC-Tests sowohl vor dem Kurs (Messzeitpunkt 1) als auch nach dem Kurs (Messzeitpunkt 2) überprüft. Die Studierenden hatten hierbei mehrere Antwortmöglichkeiten zur Verfügung. Bei neun der zehn MC-Fragen steigerten sich die Studierenden signifikant in Bezug auf die Angabe der korrekten Antwortmöglichkeit.

Um einen statistisch signifikanten Zusammenhang zu berechnen, wurden Kreuztabellen und der Chi-Quadrat-Tests bei kategorialen Variablen verwendet. Dieser gibt an, ob bei der Verteilung in der Kreuztabelle ein statistisch signifikanter Zusammenhang besteht oder nicht. Bei zwei der MC-Fragen konnte dieser Zusammenhang hergestellt werden. Insgesamt lässt sich bei den Studierenden ein klarer Wissenszuwachs zwischen Kursbeginn und -ende feststellen.

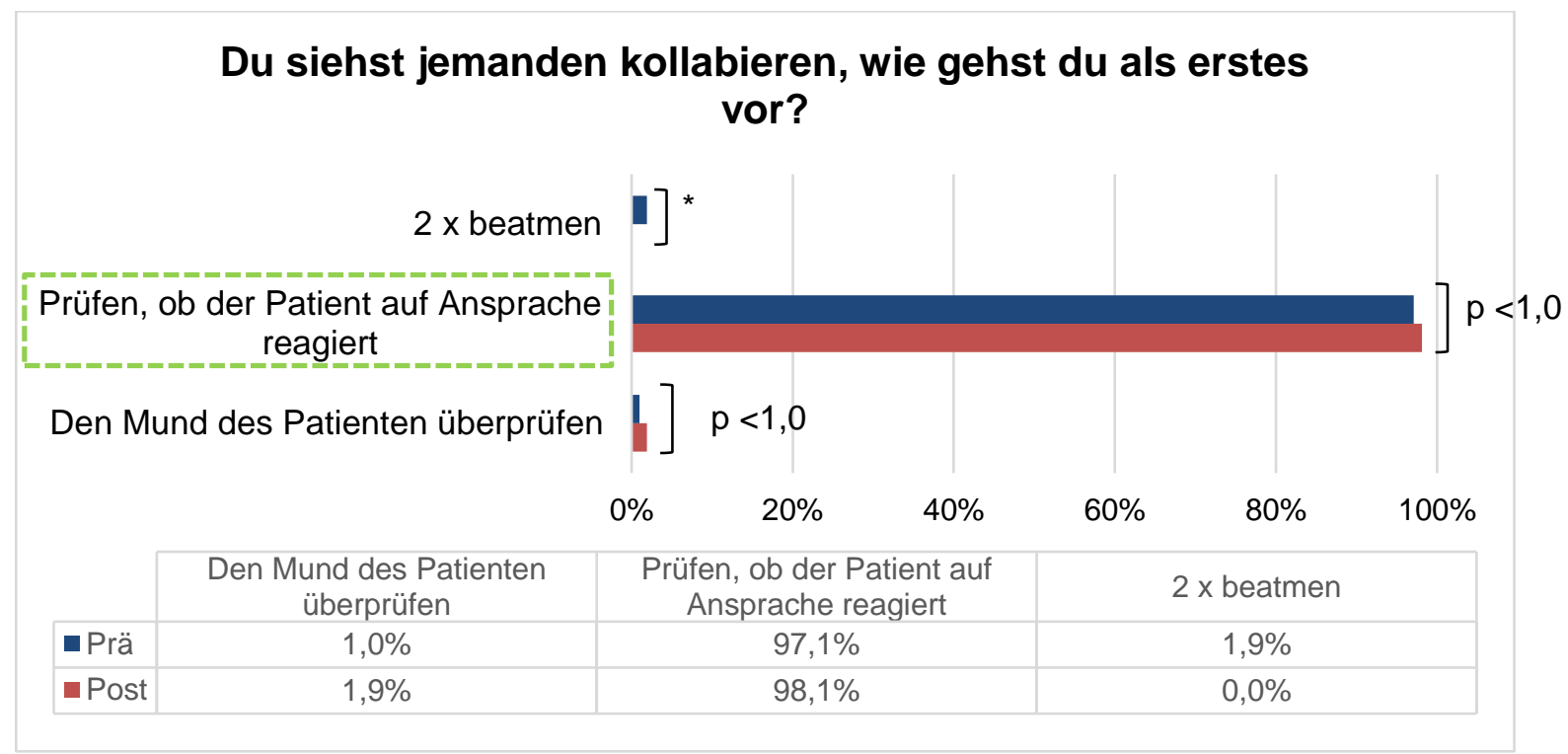

Abbildung 10: Antworten der 1. MC-Frage zum Messpunkt 1 (blau) und Messpunkt 2 (rot). Die richtige Antwort ist grün umrandet. *Hier konnte kein $p$-Wert berechnet werden, da zum Messpunkt 2 eine Konstante vorliegt.

Sowohl vor als auch nach dem Kurs beantworteten über 97\% der Studierenden die Frage richtig. Da der p-Wert mit 0,970 bei Berechnung durch den Chi-Quadrat-Test deutlich größer als das konventionelle Signifikanzniveau von 0,05 ist, besteht kein statistisch signifikanter Zusammenhang. 


\section{Wie lange versuchst du herauszufinden, ob die Person einen Kreislauf hat?}

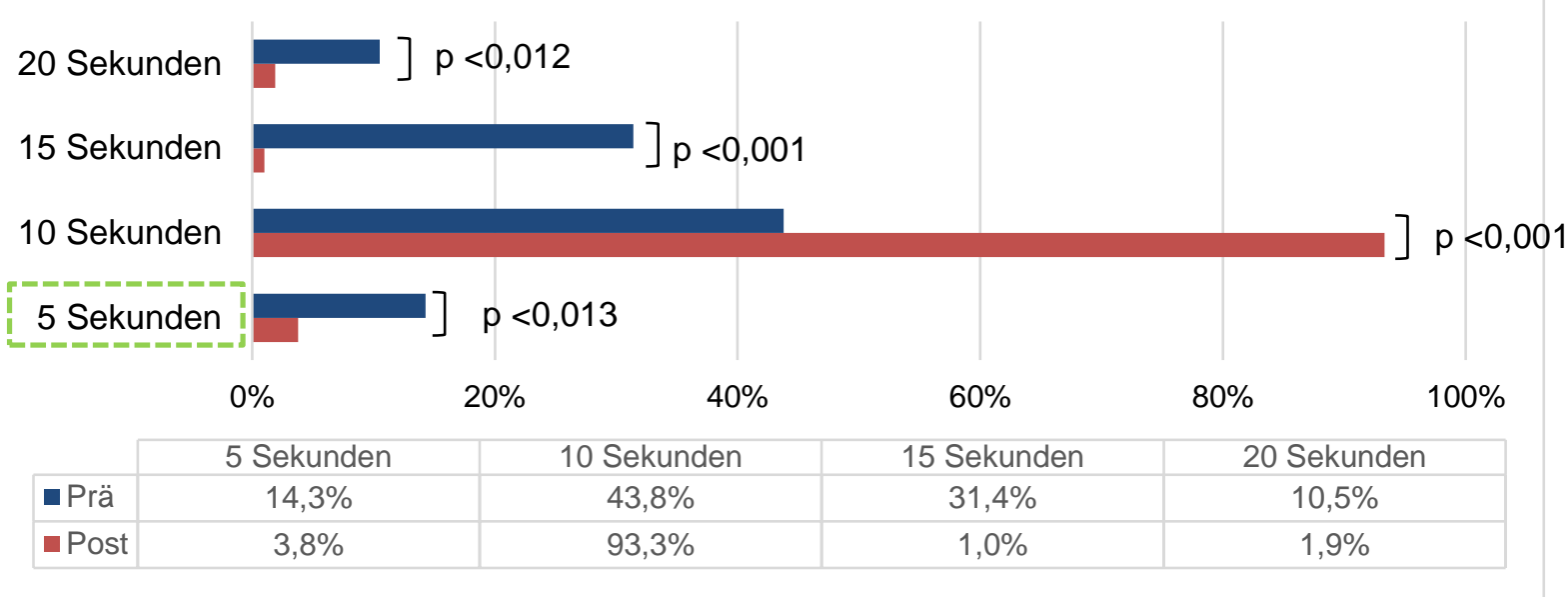

Abbildung 11: Antworten der 2. MC-Frage zum Messpunkt 1 (blau) und Messpunkt 2 (rot). Die richtige Antwort ist grün umrandet.

Wie in Abbildung 11 dargestellt, beantworteten über 90\% der Studierenden die Frage, wie lange man versucht herauszufinden, ob der Patient einen Kreislauf hat, nach dem Kurs richtig. Die Mehrheit der Studierenden hätte sich vor Kursteilnahme 15 Sekunden Zeit genommen, die Atmung zu überprüfen. Die asymptotische Signifikanz nach dem Chi-Quadrat-Test nach Pearson beträgt $p<0,570$. 


\section{Wie schnell drückt man bei der Herzdruckmassage?}

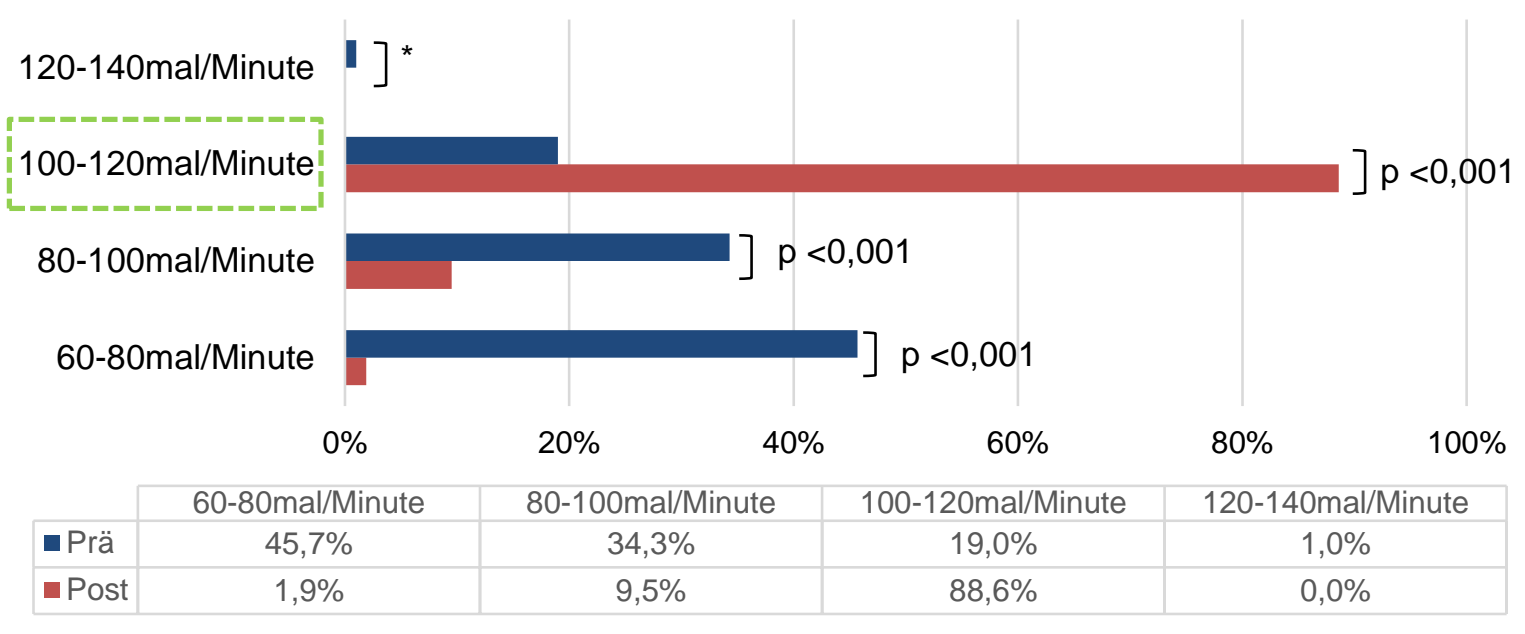

Abbildung 12: Antworten der 3. MC-Frage zum Messpunkt 1 (blau) und Messpunkt 2 (rot). Die richtige Antwort ist grün umrandet. * Hier konnte kein $p$-Wert berechnet werden, da zum Messpunkt 2 eine Konstante vorliegt.

Vor dem Kurs haben 45,7\% der Teilnehmenden eine Frequenz von 60-80/Minute für die Herzdruckmassage angegeben. Nach dem Kurs wählten die Studierenden mit 88,6\% die richtige Antwort mit 100-120mal/Minute. Hier liegt die asymptotische Signifikanz bei $p<0,993$.

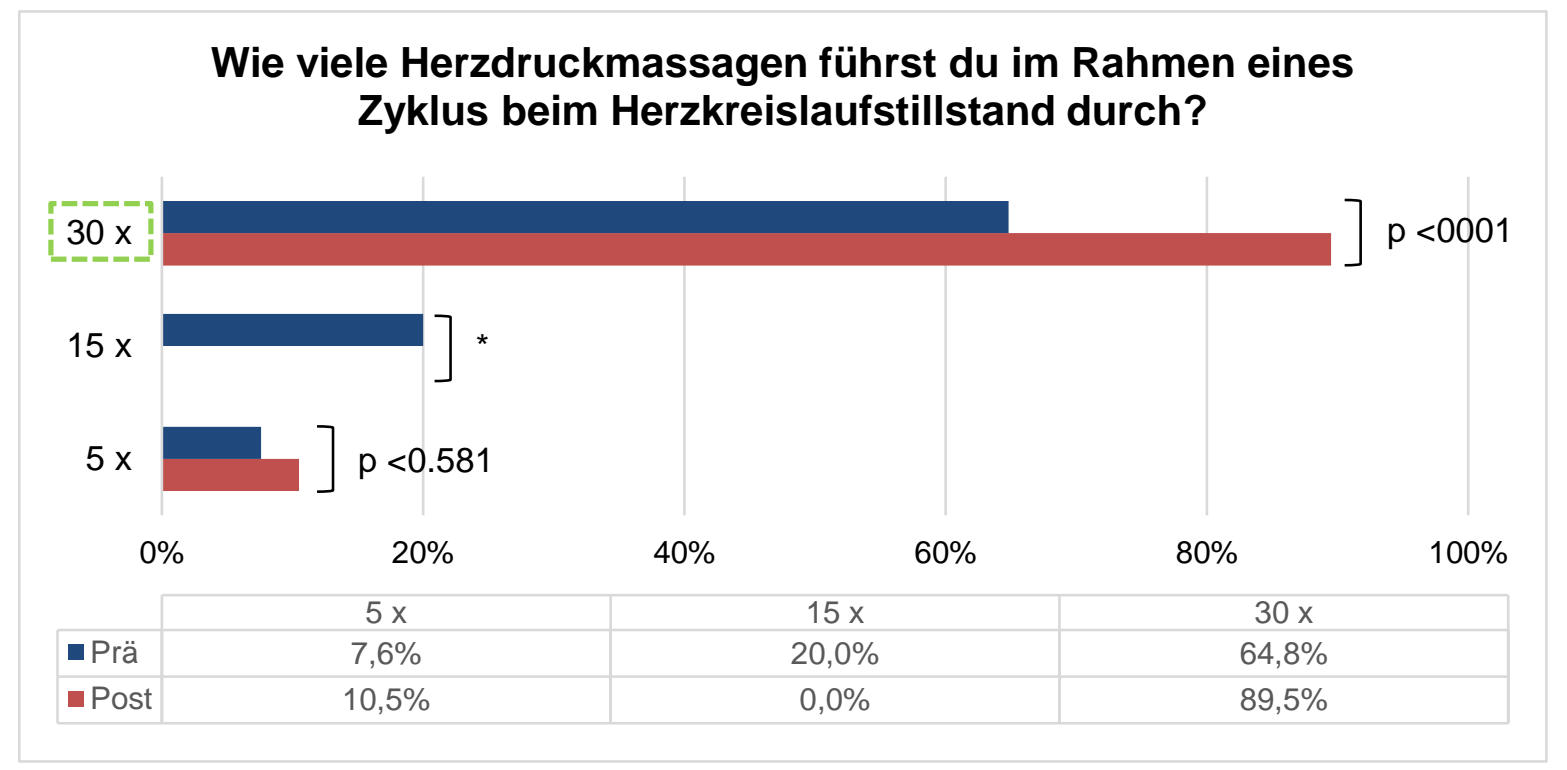

Abbildung 13. Antworten der 4. MC-Frage zum Messpunkt 1 (blau) und Messpunkt 2 (rot). Die richtige Antwort ist grün umrandet. *Hier konnte kein $p$-Wert berechnet werden, da zum Messpunkt 2 eine Konstante vorliegt. 
Fast $90 \%$ der Studierenden gaben nach dem Kurs an, 30 Herzdruckmassagen in einem Zyklus durchzuführen, während vor dem Kurs 64,8\% die richtige Antwort wählten. Die asymptotische Signifikanz nach Chi-Quadrat nach Pearson beträgt $p<0,061$.

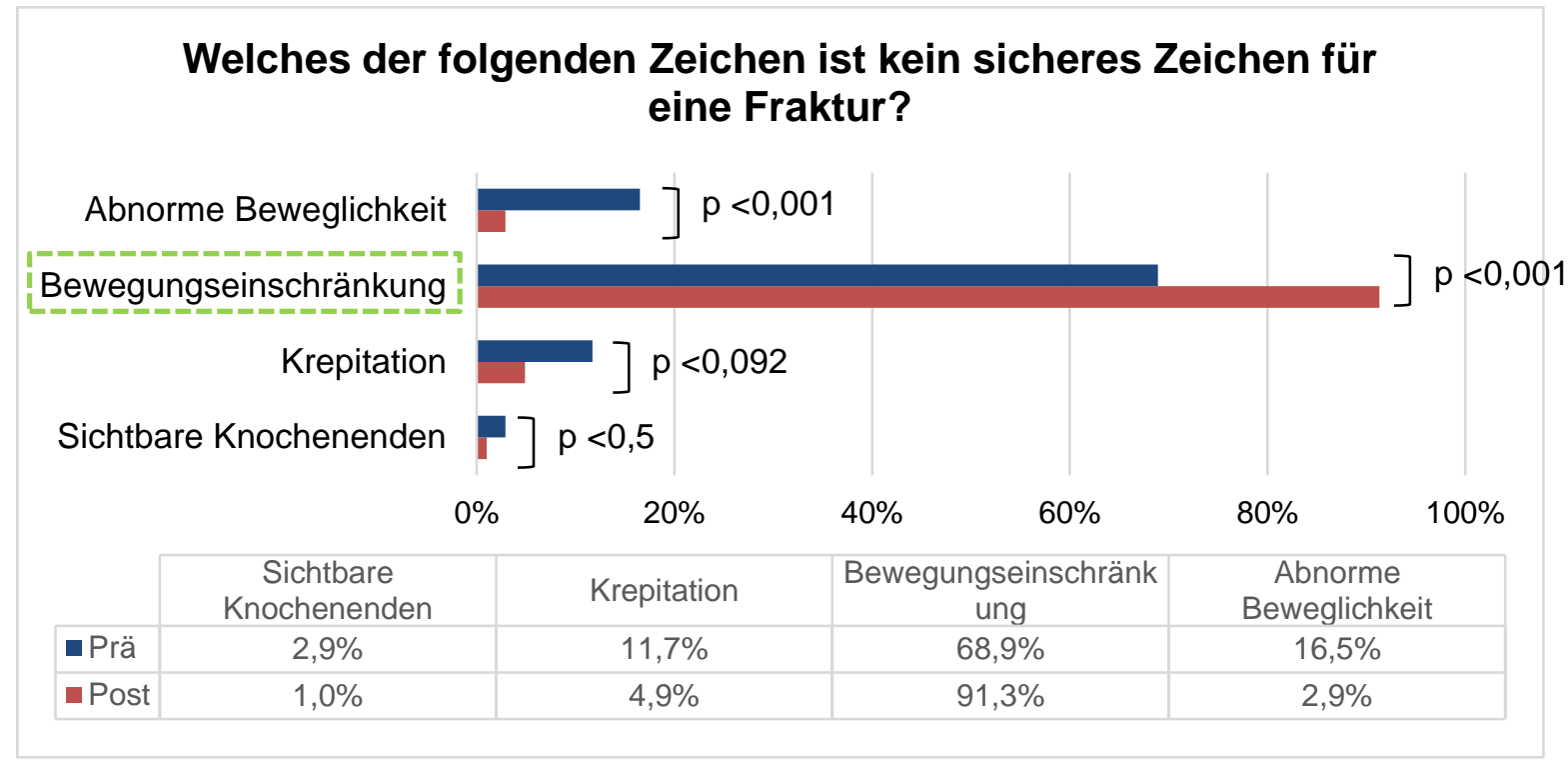

Abbildung 14: Antworten der 5. MC-Frage zum Messpunkt 1 (blau) und Messpunkt 2 (rot). Die richtige Antwort ist grün umrandet.

Nach dem Kurs konnten über 90\% der Studierenden die Antwortmöglichkeit „Bewegungseinschränkung" als die richtige Antwort identifizieren. Hier besteht ein statistisch signifikanter Zusammenhang bei $p<0,001$. 


\section{Welche der folgenden Symptome können bei einem Schlaganfall auftreten?}

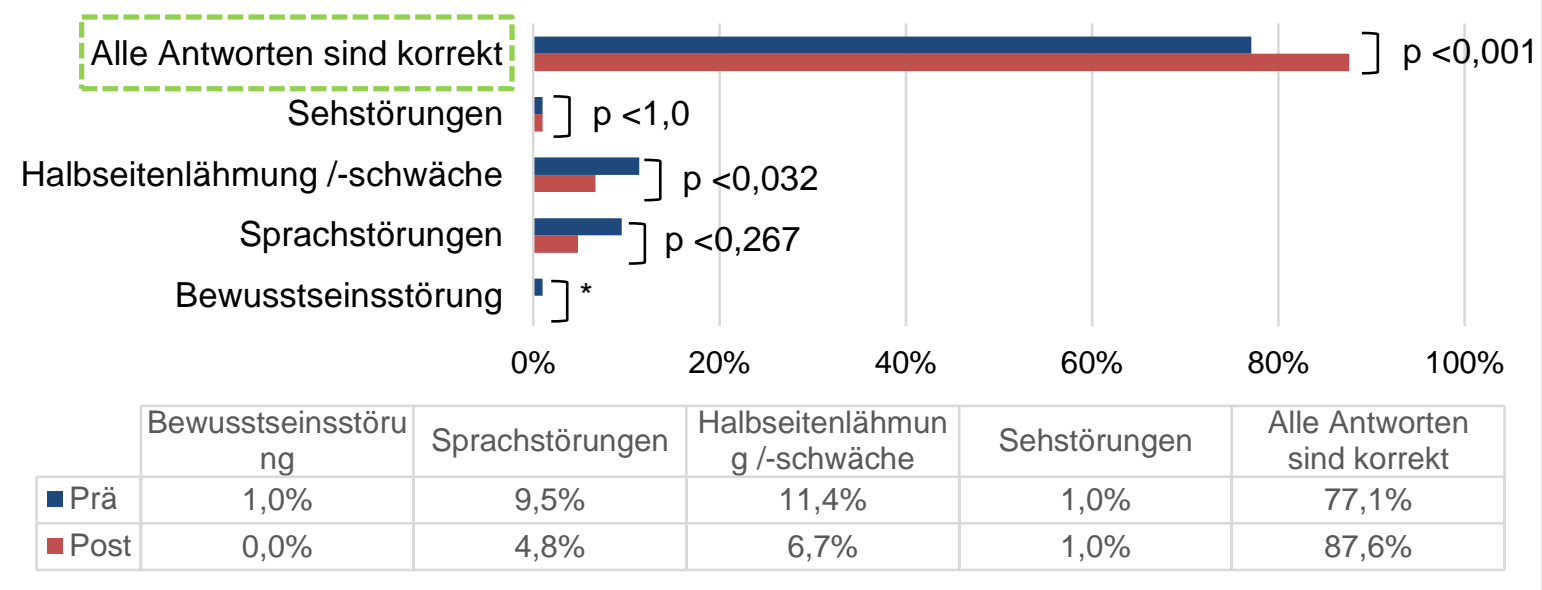

Abbildung 15: Antworten der 6. MC-Frage zum Messpunkt 1 (blau) und Messpunkt 2 (rot). Die richtige Antwort ist grün umrandet. *Hier konnte kein p-Wert berechnet werden, da zum Messpunkt 2 eine Konstante vorliegt.

Die Teilnehmenden konnten sowohl vor als auch nach dem Kurs mehrheitlich die korrekte Antwort „Alle Antworten sind korrekt“ identifizieren. Die asymptotische Signifikanz liegt bei $p<0,975$.

\section{Im Supermarkt triffst du auf einen Kunden, der über starke Brustschmerzen klagt. Wie gehst du weiter mit inm vor?}

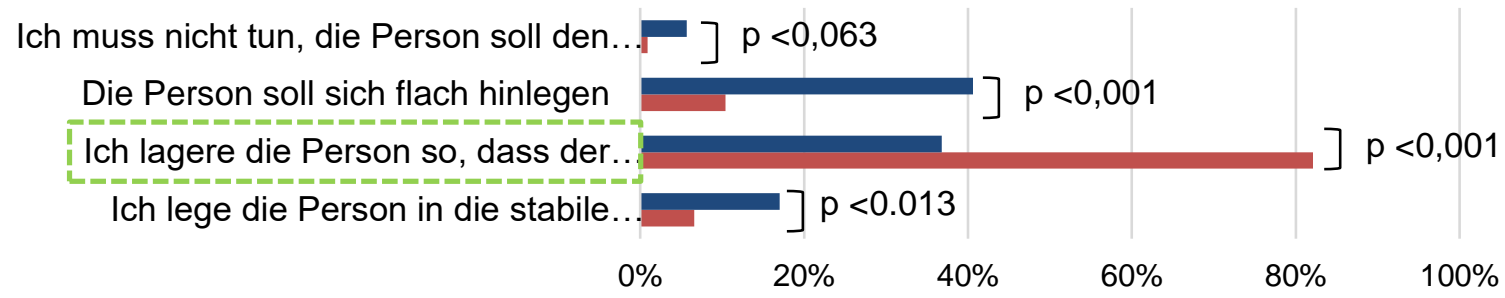

\begin{tabular}{|c|c|c|c|c|}
\hline & $\begin{array}{l}\text { Ich lege die Person in } \\
\text { die stabile Seitenlage }\end{array}$ & $\begin{array}{c}\text { Ich lagere die Person } \\
\text { so, dass der } \\
\text { Oberkörper möglichst } \\
\text { erhöht ist }\end{array}$ & $\begin{array}{l}\text { Die Person soll sich } \\
\text { flach hinlegen }\end{array}$ & $\begin{array}{c}\text { Ich muss nicht tun, die } \\
\text { Person soll den } \\
\text { Hausarzt aufsuchen }\end{array}$ \\
\hline - Prä & $17,0 \%$ & $36,8 \%$ & $40,6 \%$ & $5,7 \%$ \\
\hline Post & $6,6 \%$ & $82,1 \%$ & $10,4 \%$ & $0,9 \%$ \\
\hline
\end{tabular}

Abbildung 16: Antworten der 7. MC-Frage zum Messpunkt 1 (blau) und Messpunkt 2 (rot). Die richtige Antwort ist grün umrandet. 
Nach dem Kurs konnten 82,1\% der Studierenden die richtige Antwort identifizieren. 10,2\% wählten auch nach dem Kurs die Flachlagerung als Antwort. Laut Chi-Quadrat-Test besteht hier eine asymptotische Signifikanz mit $p<0,002$.

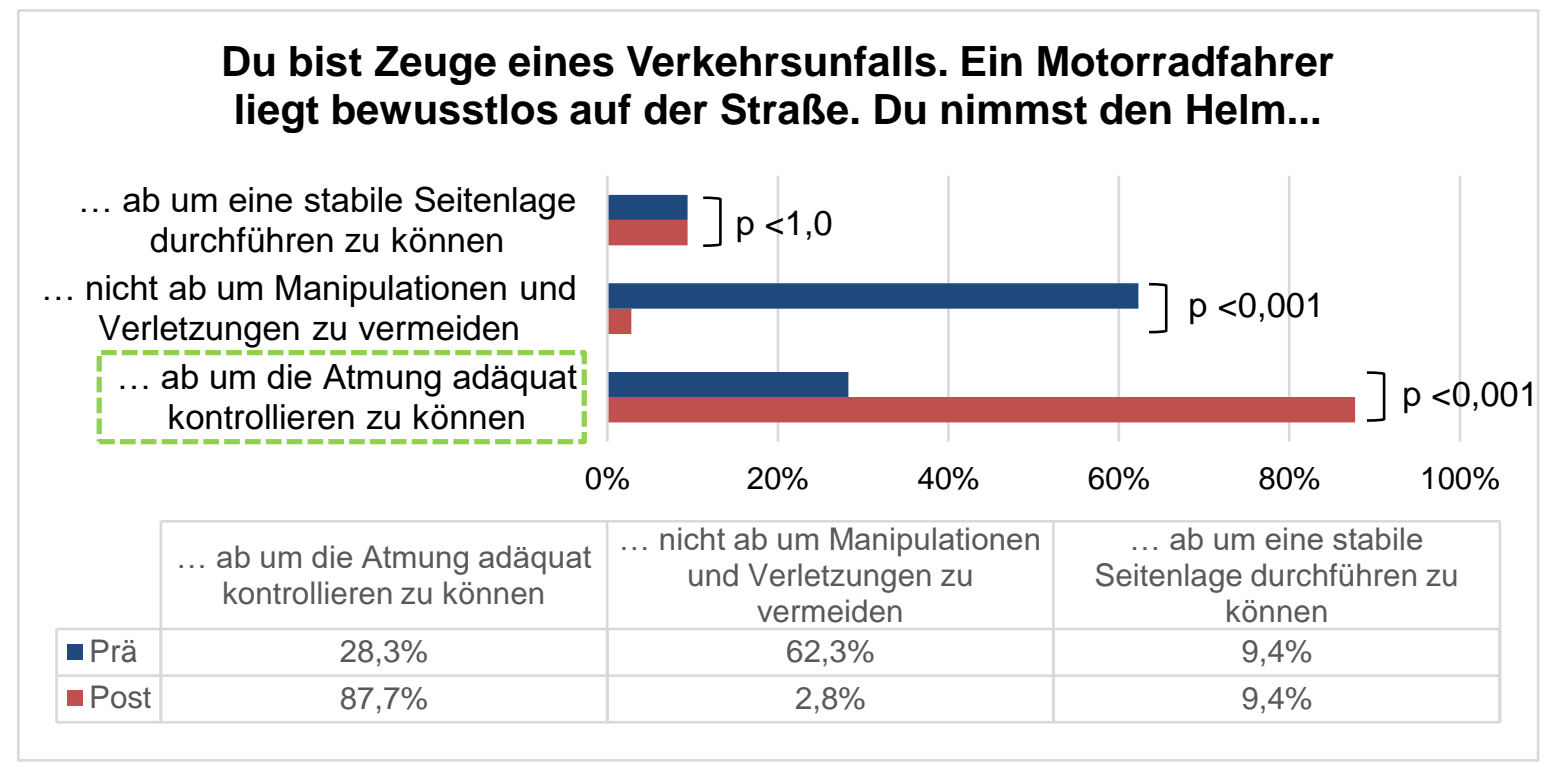

Abbildung 17: Antworten der 8. MC-Frage zum Messpunkt 1 (blau) und Messpunkt 2 (rot). Die richtige Antwort ist grün umrandet.

Vor dem Kurs nannten nur 27,7\% der Studierenden die richtige Antwort "Du nimmst den Helm ab, um die Atmung adäquat kontrollieren zu können“. Nach dem Kurs konnte diese Zahl auf 87,7\% gesteigert werden. Die Mehrheit der Studierenden gab zum Messzeitpunkt 1 vor dem Kurs an, den Helm nicht abzunehmen, um Verletzungen und Manipulationen zu vermeiden. Die asymptotische Signifikanz nach dem Chi-Quadrat-Test ist hier mit $p<0,415$ nicht gegeben. 


Du versorgst eine stark blutende Wunde, wie gehst du dabei
als erstes vor?
Keine der Aussagen trifft zu
Ka

Abbildung 18: Antworten der 9. MC-Frage zum Messpunkt 1 (blau) und Messpunkt 2 (rot). Die richtige Antwort ist grün umrandet. *Hier konnte kein p-Wert berechnet werden, da zum Messpunkt 2 eine Konstante vorliegt.

Bereits 75,5\% der Studierenden hätten zum Messzeitpunkt 1 bei einer stark blutenden Wunde einen Druckverband angelegt. Nach dem Kurs waren es 88,7\%. 8,5\% hätten nach dem Kurs die Blutung durch Abdrücken mit der Hand gestoppt. Der ChiQuadrat-Test nach Pearson ergibt hier $p<0,891$.

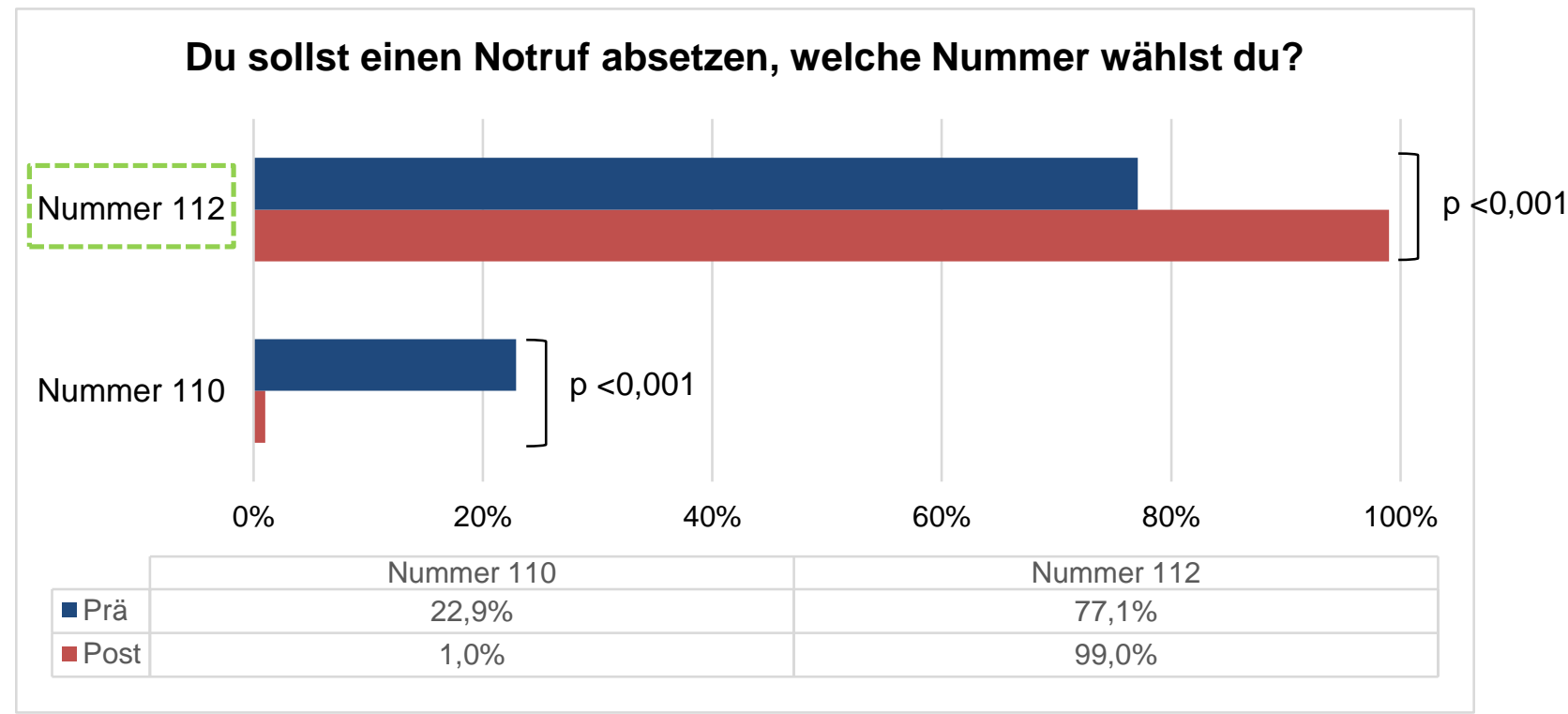

Abbildung 19: Antworten der 10. MC-Frage zum Messpunkt 1 (blau) und Messpunkt 2 (rot). Die richtige Antwort ist grün umrandet. 
Bei dieser Frage herrschte vor dem Kurs noch eine größere Unsicherheit bei den Studierenden, welche Nummer die passende für einen Notfall ist. Nach dem Kurs konnten 99\% aller Teilnehmenden die 112 als richtige Notrufnummer identifizieren. Die asymptotische Signifikanz liegt bei $p<0,584$.

\subsection{Ergebnisse der Kompetenzerfassung}

In der Kompetenzerfassung mittels der drei OSCE/OSPE-Stationen erzielten die Studienteilnehmenden an allen Stationen am Messzeitpunkt 1 jeweils im Mittel unter 50\% der möglichen Punktzahl. Im OSCE/OSPE 1 „Reanimation“ konnten maximal 18 Punkte erreicht werden. Hier wurden $42,4 \%$ vor und $80 \%$ der Punkte nach dem Kurs erreicht. Im OSCE/OSPE 2 „Stabile Seitenlage“ konnten maximal 14 Punkte erreicht werden. Hier wurden vor dem Kurs 44,1\% und nach dem Kurs 82,1\% aller Punkte erreicht. Im dritten OSCE/OSPE „Einsetzen eines AED und Anleitung eines Passanten“ konnten auch insgesamt 14 Punkte erreicht werden. Hier schafften die Studierenden vor dem Kurs im Mittel 41,9\% und nach dem Kurs im Mittel 84,2\% der zu erreichenden Punkte.

Nach dem Erste-Hilfe-Kurs, am Messzeitpunkt 2, zeigt sich an allen drei Stationen ein signifikanter Kompetenzzuwachs (Tab. 21).

\begin{tabular}{|c|c|c|c|c|}
\hline OSCE/OSPE-Station & & Messzeitpunkt 1 & Messzeitpunkt 2 & p-Wert \\
\hline \multirow[t]{2}{*}{ Ein-Helfer-Reanimation } & $\mu$ & 7,64 & 14,41 & $<0,001$ \\
\hline & $\underline{\sigma}$ & $\pm 3,62$ & $\pm 2,67$ & \\
\hline \multirow[t]{2}{*}{ Stabile Seitenlage } & $\mu$ & 6,18 & $\pm 11,5$ & $<0,001$ \\
\hline & $\underline{\sigma}$ & $\pm 2,53$ & 2,32 & \\
\hline \multirow{2}{*}{$\begin{array}{l}\text { Zwei-Helfer-Reanimation, } \\
\text { Einsetzen AED und Anlei- } \\
\text { tung Passant }\end{array}$} & $\mu$ & 5,87 & 11,79 & $<0,001$ \\
\hline & $\underline{\sigma}$ & $\pm 3,085$ & $\pm 2,183$ & \\
\hline
\end{tabular}

Tabelle 21: Erreichte absolute Punktzahl an den 3 OSCE/OSPE-Stationen zum Messzeitpunkt 1 und 2 als Mittelwert $(=\mu)$ und Standardabweichung $(=\sigma)$, sowie die Signifikanz. 


\subsubsection{OSCE/OSPE 1 - Ein-Helfer-Reanimation}

Bei der Detailauswertung der Einzelitems an der jeweiligen OSCE/OSPE-Station zeigen sich ebenfalls signifikante Kompetenzsteigerungen für die einzelnen Items (Tab. 22). In der OSCE/OSPE-Station „Reanimation“ zeigten sich deutliche Unterschiede im Prä-Post-Vergleich. So haben nur 3,1\% der Studienteilnehmenden zum Messzeitpunkt 1 eine Eigengefährdung ausgeschlossen, am Messzeitpunkt 2 63,1\% $(p<0,001)$. Ein Notruf wurde zum Messzeitpunkt 1 von 47,5\% der Teilnehmenden abgesetzt, am Messzeitpunkt 2 von 81,3\% ( $p<0,001)$.

\begin{tabular}{|c|c|c|c|c|}
\hline \multicolumn{2}{|l|}{ Maßnahme } & $\begin{array}{c}\text { Mess- } \\
\text { zeitpunkt } \\
1\end{array}$ & $\begin{array}{l}\text { Mess- } \\
\text { zeitpunkt } \\
2\end{array}$ & p-Wert \\
\hline \multicolumn{2}{|c|}{ Eigengefährdung ausgeschlossen } & $3,1 \%$ & $63,1 \%$ & $<0,001$ \\
\hline \multicolumn{2}{|c|}{ Patient angesprochen } & $71,3 \%$ & $95,0 \%$ & $<0,001$ \\
\hline \multicolumn{2}{|l|}{ Patient geschüttelt } & $50,0 \%$ & $86,9 \%$ & $<0,001$ \\
\hline \multicolumn{2}{|l|}{ Um Hilfe gerufen } & $52,5 \%$ & $66,9 \%$ & 0,008 \\
\hline \multicolumn{5}{|c|}{ Überprüfung der Atemwege: } \\
\hline \multicolumn{2}{|l|}{ Kopf rekliniert } & $22,5 \%$ & $84,4 \%$ & $<0,001$ \\
\hline \multicolumn{2}{|l|}{ Kinn vorgezogen } & $15,1 \%$ & $58,5 \%$ & $<0,001$ \\
\hline \multicolumn{5}{|l|}{ Atemkontrolle: } \\
\hline \multicolumn{2}{|c|}{ Sehen, hören, fühlen korrekt durchgeführt } & $51,9 \%$ & $96,3 \%$ & $<0,001$ \\
\hline \multicolumn{2}{|c|}{10 Sekunden die Atmung kontrolliert } & $20,0 \%$ & $81,3 \%$ & $<0,001$ \\
\hline \multicolumn{2}{|l|}{ Notruf abgesetzt } & $47,5 \%$ & $81,3 \%$ & $<0,001$ \\
\hline \multicolumn{5}{|l|}{ Reanimation: } \\
\hline \multicolumn{2}{|l|}{ Richtiger Druckpunkt } & $61,9 \%$ & $86,3 \%$ & $<0,001$ \\
\hline \multicolumn{2}{|c|}{ Korrekte Hand- und Körperhaltung } & $58,8 \%$ & $97,5 \%$ & $<0,001$ \\
\hline \multicolumn{2}{|c|}{30 Herzdruckmassagen durchgeführt } & $61,3 \%$ & $96,3 \%$ & $<0,001$ \\
\hline \multirow{2}{*}{$\begin{array}{l}\text { Korrekte Frequenz } \\
(100-120 \mathrm{bpm})\end{array}$} & 1 Punkt & $26,9 \%$ & $21,3 \%$ & \multirow[t]{2}{*}{$<0,001$} \\
\hline & 2 Punkte & $26,9 \%$ & $70,0 \%$ & \\
\hline Korrekte Drucktiefe & 1 Punkt & $28,7 \%$ & $33,8 \%$ & $<0,001$ \\
\hline
\end{tabular}




\begin{tabular}{|l|l|l|l|l|}
\hline$(5$ bis max. $6 \mathrm{~cm})$ & 2 Punkte & $23,8 \%$ & $52,5 \%$ & \\
\hline Entlastung & & $43,1 \%$ & $66,3 \%$ & $<0,001$ \\
\hline
\end{tabular}

Tabelle 22: Kompetenzerfassung der OSCE/OSPE-Station „Ein-Helfer-Reanimation“ - dargestellt sind für alle Items der Checkliste zu beiden Messzeitpunkten jeweils die erreichten Prozent sowie die Signifikanz.

Die Qualitätsindikatoren einer adäquaten Reanimation sind insbesondere die Druckfrequenz, Drucktiefe und Entlastung. Hier konnte zwischen Messzeitpunkt 1 und 2 eine signifikante Verbesserung gezeigt werden $(p<0,001)$ (Abb. 20).

30 Herzdruckmassagen durchgeführt

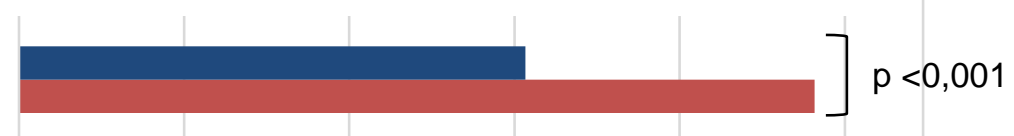

Korrekte Drucktiefe

Korrekte Frequenz

Richtiger Druckpunkt

Entlastung

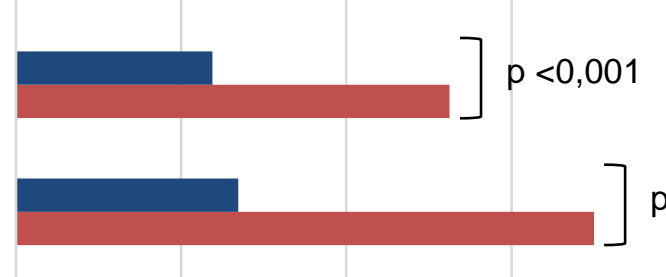

$p<0,001$

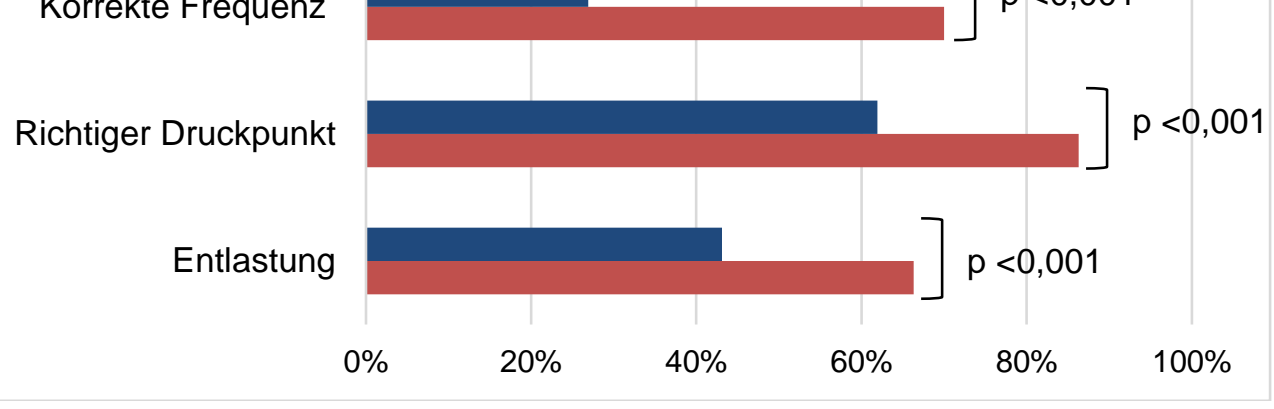

Abbildung 20: Schlüsselmaßnahmen der Reanimationen - dargestellt sind die Ergebnisse der Studienteilnehmenden in Prozent zum Messzeitpunkt 1 (blau) und Messzeitpunkt 2 nach dem Kurs (rot) für die fünf Einzelitems sowie die Signifikanz.

\subsubsection{OSCE/OSPE 2 - Stabile Seitenlage}

Auch in der OSCE/OSPE-Station "Stabile Seitenlage" zeigten sich deutliche Unterschiede im Prä-Post-Vergleich (Tab. 23). Hervorzuheben ist, dass sich die Studierenden bei den Maßnahmen der Atemkontrolle mit Sehen, Hören und Fühlen (prä = $81,8 \%$, post $=97,5 \%, p<0,001$ ) und sich 10 Sekunden dafür Zeit nehmen (prä = $28,3 \%$, pos $t=87,4 \%, p<0,001)$ nach dem Kurs signifikant gesteigert haben. Auch die Schlüsselmaßnahmen der Stabilen Seitenlage mit Kopf überstrecken (prä = $59,1 \%$, post $=95,0 \%, p<0,001$ ) und Mund öffnen (prä $=29,1 \%$, post $=82,9 \%$, $p<0,001)$ konnten nach Kursteilnahme signifikant verbessert werden. 


\begin{tabular}{|l|c|c|c|}
\hline Maßnahme & $\begin{array}{c}\text { Mess- } \\
\text { zeitpunkt }\end{array}$ & $\begin{array}{c}\text { Mess- } \\
\text { zeitpunkt }\end{array}$ & p-Wert \\
\hline Eigengefährdung ausgeschlossen & $\mathbf{1}$ & $\mathbf{2}$ & \\
\hline Patient angesprochen & $3,1 \%$ & $60,4 \%$ & $<0,001$ \\
\hline Patient geschüttelt & $64,8 \%$ & $96,2 \%$ & $<0,001$ \\
\hline Um Hilfe gerufen & $54,1 \%$ & $88,1 \%$ & $<0,001$ \\
\hline Überprüfung der Atemwege: & $41,5 \%$ & $54,7 \%$ & 0,016 \\
\hline Kopf rekliniert & & & \\
\hline Kinn vorgezogen & $20,8 \%$ & $73,0 \%$ & $<0,001$ \\
\hline Atemkontrolle: & $11,9 \%$ & $67,3 \%$ & $<0,001$ \\
\hline Sehen, hören, fühlen korrekt durchgeführt & $81,8 \%$ & $97,5 \%$ & $<0,001$ \\
\hline 10 Sekunden die Atmung kontrolliert & $28,3 \%$ & $87,4 \%$ & $<0,001$ \\
\hline Notruf abgesetzt & $49,7 \%$ & $81,1 \%$ & $<0,001$ \\
\hline Durchführung der Seitenlage: & & & \\
\hline Lagerung in Rückenlage & $89,3 \%$ & $98,1 \%$ & 0,001 \\
\hline Stabile Seitenlage & $59,1 \%$ & $95,0 \%$ & $<0,001$ \\
\hline Überstrecken Kopf, & $5,7 \%$ & $5,1 \%$ & $<0,001$ \\
\hline Öffnung Mund & $29,1 \%$ & $82,9 \%$ & \\
\hline
\end{tabular}

Tabelle 23: Kompetenzerfassung OSCE/OSPE-Station "Stabile Seitenlage“ - dargestellt sind für alle Items der Checkliste zu beiden Messzeitpunkten jeweils die erreichten Prozent sowie die Signifikanz.

Die Mehrheit der Studierenden beherrschten vor dem Kurs keine der Schlüsselmaßnahmen der Stabilen Seitenlage wie das Überstrecken des Kopfes und das Öffnen des Mundes ausreichend. Nach dem Kurs zeigte sich wie in Abbildung 21 dargestellt ein signifikanter Zuwachs der Kompetenzen. 


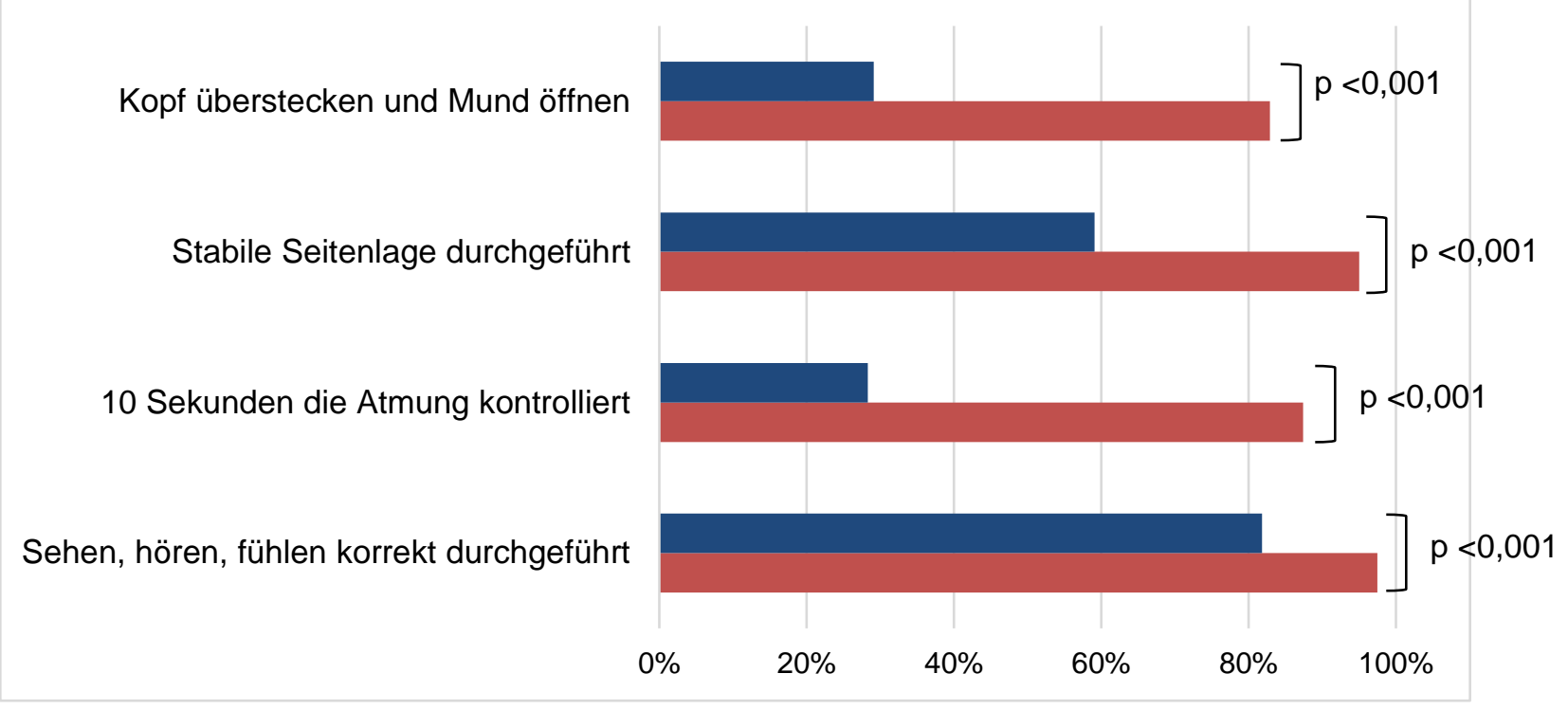

Abbildung 21: Schlüsselmaßnahmen der Stabilen Seitenlage - dargestellt sind die Ergebnisse der Studienteilnehmenden in Prozent zum Messzeitpunkt 1 vor dem Kurs (blau) und Messzeitpunkt 2 nach dem Kurs (rot) für die vier Einzelitems sowie die Signifikanz.

\subsubsection{OSCE/OSPE 3 - Zwei-Helfer-Reanimation, Einsetzen des AED und An- leitung eines Passanten}

Auch an der dritten OSCE/OSPE-Station „Zwei-Helfer-Reanimation, Einsetzen eines AED und Anleitung eines Passanten“ waren alle Ergebnisse hoch signifikant (Tab. 24). Besonders fällt auf, dass sich die Studierenden in der Wiederaufnahme der CPR-Maßnahmen nach dem Kurs signifikant steigern konnten (prä $=45,1 \%$, post $=93,4 \%, p<0,001)$ und somit die No-Flow-Time stark reduziert wurde.

\begin{tabular}{|l|c|c|c|c|}
\hline Maßnahme & & $\begin{array}{c}\text { Mess- } \\
\text { zeitpunkt }\end{array}$ & $\begin{array}{c}\text { Mess- } \\
\text { zeitpunkt }\end{array}$ & p-Wert \\
\cline { 1 - 5 } & & $\mathbf{1}$ & $\mathbf{2}$ & \\
\hline Eigengefährdung ausgeschlossen & $7,7 \%$ & $62,6 \%$ & $<0,001$ \\
\hline Startet mit der CPR & & $86,8 \%$ & $96,7 \%$ & 0,035 \\
\hline Bindet Passanten ein & & $67,0 \%$ & $97,8 \%$ & $<0,001$ \\
Setzt den AED ein & 1 Punkt & $2,2 \%$ & - & $<0,001$ \\
& 2 Punkte & $73,6 \%$ & $100 \%$ & \\
\hline
\end{tabular}




\begin{tabular}{|l|l|c|c|c|}
\hline Befolgt Anweisungen des AEDs korrekt & $64,8 \%$ & $97,8 \%$ & $<0,001$ \\
\cline { 1 - 4 } Beachtet korrekte Reihenfolge & 1 Punkt & $6,6 \%$ & $1,1 \%$ & $<0,001$ \\
\cline { 2 - 5 } & 2 Punkte & $48,4 \%$ & $83,5 \%$ & \\
\hline Gibt Kommando „Alle weg“ & $3,3 \%$ & $74,7 \%$ & $<0,001$ \\
\hline Kontrolle, dass keiner Patienten berührt & $4,4 \%$ & $72,5 \%$ & $<0,001$ \\
\hline Gibt Kommando: „Schock“ & $1,1 \%$ & $56,0 \%$ & $<0,001$ \\
\hline Löst Schock aus & $49,5 \%$ & $86,8 \%$ & $<0,001$ \\
\hline CPR sofort weiter fortgeführt & $45,1 \%$ & $93,4 \%$ & $<0,001$ \\
\hline
\end{tabular}

Tabelle 24: Kompetenzerfassung OSCE/OSPE-Station „Zwei-Helder-Reanimation, Einsetzen eines AED und Anleitung eines Passanten“-dargestellt sind für alle Items der Checkliste zu beiden Messzeitpunkten jeweils die erreichten Prozent sowie die Signifikanz.

Vor dem Kurs setzten nur 73,6\% der Studierenden einen AED ein und weniger als die Hälfte beachtete die korrekte Reihenfolge der Anweisungen des AED. Nur 49,5\% lösten einen Schock aus und beachteten dabei die Sicherheitsmaßnahmen bei der Schockabgabe. Insgesamt konnten die Schlüsselmaßnahmen dieser Station vor dem Kurs nicht ausreichend von den Studierenden erreicht werden. Nach dem Kurs gab es in allen Items eine signifikante Steigerung.

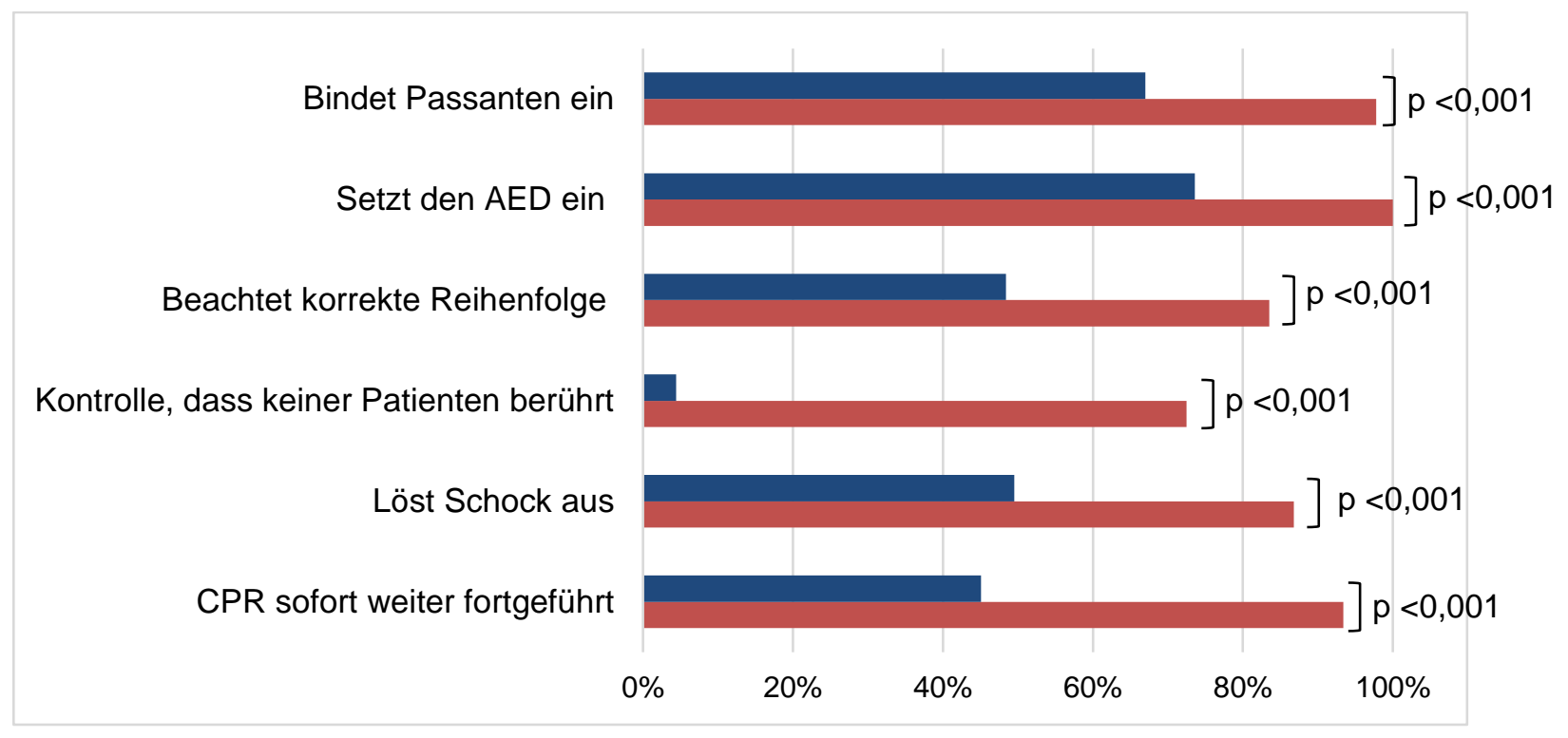

Abbildung 22: Schlüsselmaßnahmen bei der Zwei-Helfer-Reanimation und Einsatz eines AED - dargestellt sind die Ergebnisse der Studienteilnehmenden in Prozent zum Messzeitpunkt 1 vor dem Kurs (blau) und Messzeitpunkt 2 nach dem Kurs (rot) für die sechs Einzelitems sowie die Signifikanz. 


\subsection{Ergebnisse Kursevaluation}

Insgesamt war die Evaluation des Erste-Hilfe-Kurses durchweg sehr positiv. Zunächst wurde Allgemeines zum Kurs anhand einer 5-Punkt Likert-Skala ( $1=$ „sehr

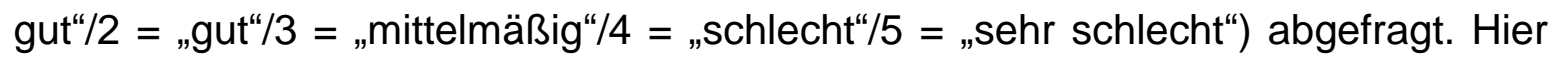
war die Zufriedenheit der Studierenden sehr hoch und reichte von 1,1 $\pm 0,353$ bis $1,35 \pm 0,657$ (MD $\pm S D$ ). Über $80 \%$ der Studierenden bewerteten den Kurs sehr gut, es gab keine mittelmäßigen, schlechten oder sehr schlechten Bewertungen.

\begin{tabular}{|l|c|c|c|}
\hline Frage & MW \pm SD & Min & Max \\
\hline Wie bewertet ihr den Kurs insgesamt? & $1,14 \pm 0,350$ & 1 & 2 \\
\hline Wie war die Atmosphäre im Kurs? & $1,13 \pm 0,421$ & 1 & 4 \\
\hline $\begin{array}{l}\text { Wie war das Auftreten/die Kommunikation des Instruk- } \\
\text { tors? }\end{array}$ & $1,10 \pm 0,353$ & 1 & 3 \\
\hline \begin{tabular}{l} 
Wie habt ihr Euch in unseren Räumlichkeiten gefühlt? \\
\hline
\end{tabular} & $1,35 \pm 0,657$ & 1 & 5 \\
\hline
\end{tabular}

Tabelle 25: Tabellarische Darstellung der Evaluation zu den allgemeinen Kursinhalten. $M W=$ arithmetischer Mittelwert, SD = Standardabweichung, Min = Minimum, Max = Maximum.

\section{Allgemeines zum Kurs}

Wie habt ihr Euch in unseren Räumlichkeiten gefühlt?

Wie war das Auftreten/die Kommunikation des Instruktors?

Wie war die Atmosphäre im Kurs?

Wie bewertet Ihr den Kurs insgesamt?
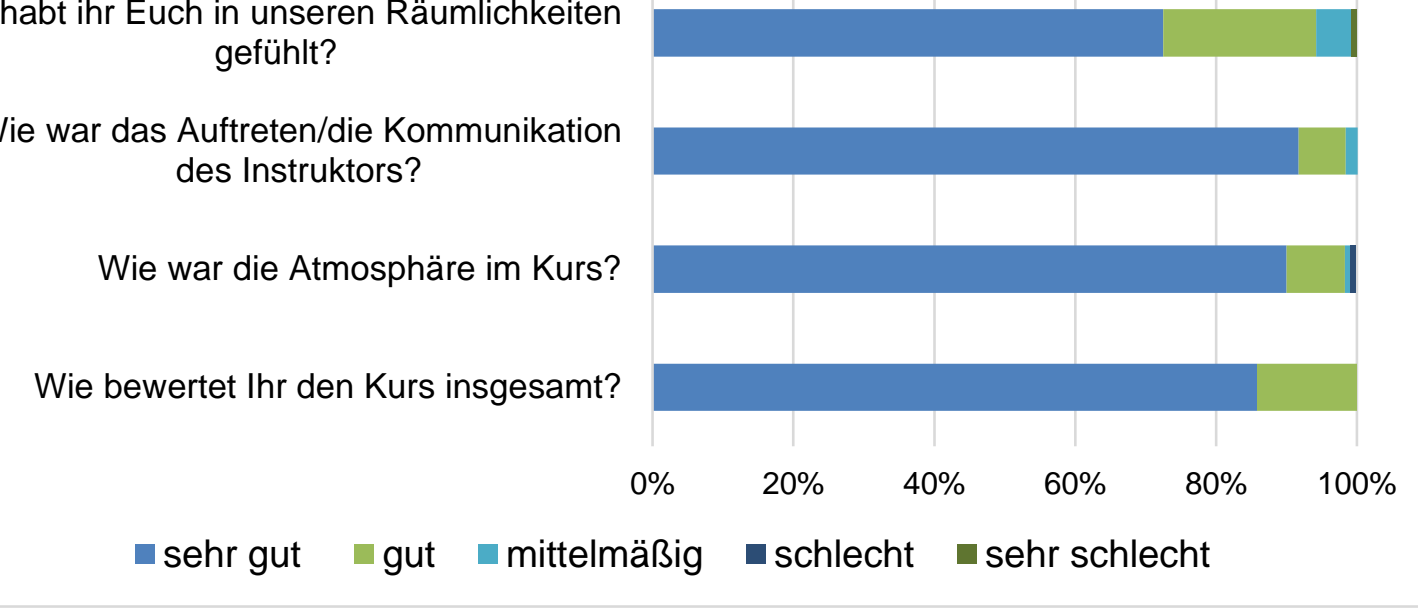

Abbildung 23: Grafische Darstellung der Kursevaluationen zu den allgemeinen Kursinhalten. 
Dann folgten Fragen zur generellen Struktur und dem Aufbau des Kurses, diese wurden auch mit einer 5-Punkte-Likert-Skala ( 1 = „zu lang“/2 = „länger als geplant“/3 $=$, optimal“/4 = „kürzer als geplant“/5 = „zu kurz“) abgefragt. Auch hier zeigte sich ein positives Ergebnis: die Mehrheit der Studierenden sahen Dauer des Kurses, Praxisanteil, Theorieanteil und die Pausen als optimal an. Insbesondere der hohe Praxisanteil mit 6 von 9 UE wurde von den Studierenden wertgeschätzt.

\begin{tabular}{|l|c|c|c|}
\hline Frage & MW \pm SD & Min & Max \\
\hline Die Dauer des Kurses war insgesamt & $2,45 \pm 0,620$ & 1 & 4 \\
\hline Der Praxisanteil war & $3,05 \pm 0,339$ & 1 & 4 \\
\hline Theorieanteil war & $2,68 \pm 0,565$ & 1 & 4 \\
\hline Die Pausen waren & $2,89 \pm 0,515$ & 1 & 4 \\
\hline
\end{tabular}

Tabelle 26: Tabellarische Darstellung der Kursevaluationen zu Struktur und Aufbau des Kurses, MW = arithmetischer Mittelwert, SD = Standardabweichung, Min = Minimum, Max = Maximum.

\section{Struktur und Aufbau des Kurses}

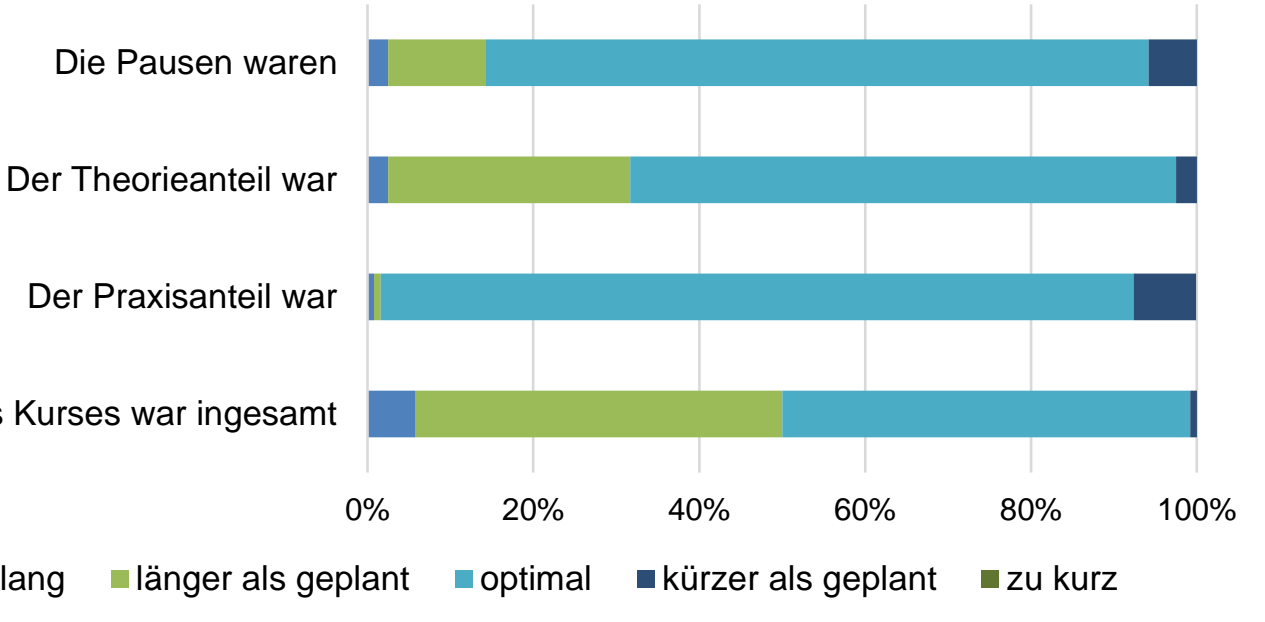

Abbildung 24: Grafische Darstellung der Kursevaluationen zu Struktur und Aufbau des Erste-Hilfe-Kurses. 
Mit einer 5-Punkte-Likert-Skala (1 = „triftt zu“/2 = „trifft eher zu“/3 = „teils-teils“/4 = „trifft eher nicht zu“/ 5 = „trifft nicht zu“) wurden noch einmal einzelne Aspekte des Erste-Hilfe-Trainings abgefragt. Hier zeigte sich, dass die Studierenden den Kurs durchgängig als wichtig und interessant einschätzten. Des Weiteren war der Kurs für die Vorklinikstudierenden umsetzbar.

\begin{tabular}{|l|c|l|l|}
\hline Frage & MW \pm SD & Min & Max \\
\hline Das Erste-Hilfe Training war theoretisch interessant & $1,24 \pm 0,485$ & 1 & 3 \\
\hline Das Erste-Hilfe Training war notwendig & $1,18 \pm 0,449$ & 1 & 3 \\
\hline $\begin{array}{l}\text { Das Erste-Hilfe Training war praktisch für mich persön- } \\
\text { lich umsetzbar }\end{array}$ & $1,15 \pm 0,359$ & 1 & 2 \\
\hline $\begin{array}{l}\text { Das Erste-Hilfe Training war meinen Vorstellungen } \\
\text { entsprechend }\end{array}$ & $1,28 \pm 0,624$ & 1 & 5 \\
\hline
\end{tabular}

Tabelle 27: Tabellarische Darstellung der Kursevaluationen zu einzelnen Aspekten des Erste-Hilfe-Kurses, MW $=$ arithmetischer Mittelwert, SD = Standardabweichung, Min = Minimum, Max = Maximum.

\section{Aspekte des Erste-Hilfe-Kurses}

Das Erste-Hilfe Training war meinen Vorstellungen entsprechend

Das Erste-Hilfe Training war praktisch für mich persönlich umsetzbar

Das Erste-Hilfe Training war notwendig

Das Erste-Hilfe Training war theoretisch interessant

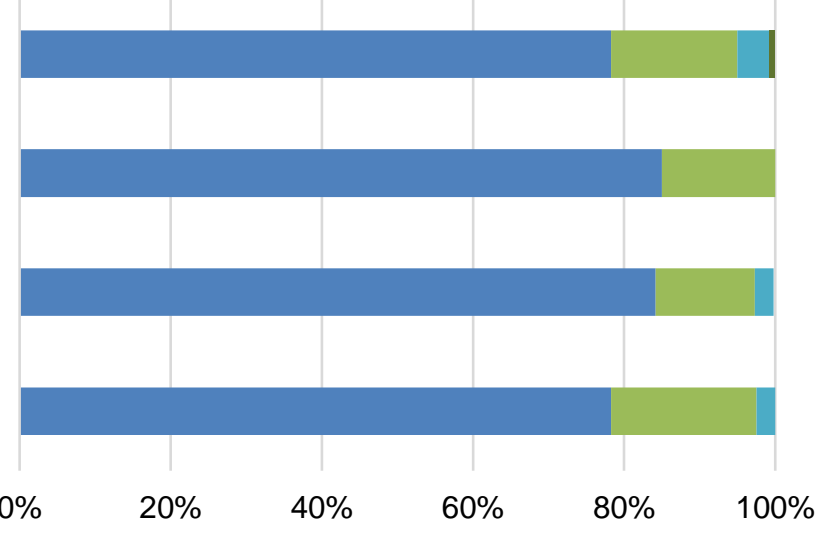

Trifft zu $\quad$ Trifft eher zu $\quad$ Teils-teils $\quad \square$ Trifft eher nicht zu $\quad \square$ Trifft nicht zu

Abbildung 25: Grafische Darstellung der Kursevaluationen zu einzelnen Aspekten des Erste-Hilfe-Kurses. 
Auch die Instruktor:innen wurden mit einer 5-Punkte-Likert-Skala (1 = „trifft zu“/2 = „trifft eher zu“/3 = „teils-teils“/4 = „trifft eher nicht zu“/5 = „trifft nicht zu“) evaluiert. Hier zeigte sich eine durchweg positive Evaluation der Peer-Dozierenden durch die Studierenden.

\begin{tabular}{|l|c|l|l|}
\hline Frage & MW \pm SD & Min & Max \\
\hline $\begin{array}{l}\text { Der/Die Instruktor:in hat klar und deutlich gesprochen } \\
\begin{array}{l}\text { Der/Die Instruktor:in geht angemessen auf Anregun- } \\
\text { gen und Fragen ein }\end{array}\end{array}$ & $1,06 \pm 0,338$ & 1 & 4 \\
\hline $\begin{array}{l}\text { Der/Die Instruktor:in erklärt Sachverhalte und Zu- } \\
\text { sammenhänge verständlich }\end{array}$ & $1,08 \pm 0,279$ & 1 & 2 \\
\hline $\begin{array}{l}\text { Der/Die Instruktor:in vermittelt den Stoff in einem an- } \\
\text { genehmen Tempo }\end{array}$ & $1,17 \pm 0,459$ & 1 & 4 \\
\hline
\end{tabular}

Tabelle 28: Tabellarische Darstellung der Kursevaluationen zu den Instruktor:innen, MW = arithemtischer Mittelwert, SD = Standardabweichung, Min = Minimum, Max = Maximum.

\section{Instruktor:innen}

Der/Die Instruktor:in vermittelt den Stoff in einem angenehmen Tempo

Der/Die Instruktor:in erklärt Sachverhalte und Zusammenhänge verständlich

Der/Die Instruktor:in geht angemessen auf Anregungen und Fragen ein

Der/Die Instruktor:in hat klar und deutlich gesprochen

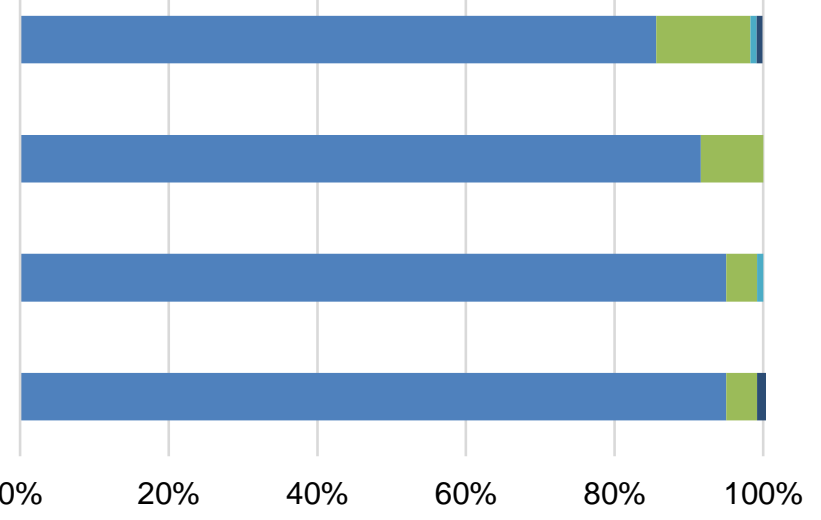

$\square$ Trifft zu $\quad$ Trifft eher zu $\quad$ Teils-teils $\quad$ Trifft eher nicht zu $\quad$ Trifft nicht zu

Abbildung 26: Grafische Darstellung der Kursevaluationen zu den Instruktor:innen. 
Bei den Räumlichkeiten und dem Material wurde besonders die Anzahl von acht Teilnehmenden in einer Kleingruppe positiv evaluiert. Zusätzlich schätzten die Studierenden, ausreichend Material zum Üben und die Größe des Kursraumes.

\begin{tabular}{|l|c|l|l|}
\hline Frage & MW \pm SD & Min & Max \\
\hline Der Kursraum war groß genug & $1,23 \pm 0,576$ & 1 & 5 \\
\hline Die Anzahl der Teilnehmer:innen war angemessen & $1,04 \pm 0,201$ & 1 & 2 \\
\hline Es gab ausreichend Material zum Üben & $1,15 \pm 0,403$ & 1 & 3 \\
\hline
\end{tabular}

Tabelle 29: Tabellarische Darstellung der Kursevaluationen zu Räumlichkeiten und Material, MW = arithmetischer Mittelwert, SD = Standardabweichung, Min = Minimum, Max = Maximum.

\section{Räumlichkeiten und Material}

Es gab ausreichend Material zum Üben

Die Anzahl der Teilnehmer:innen war angemessen

Der Kursraum war groß genug
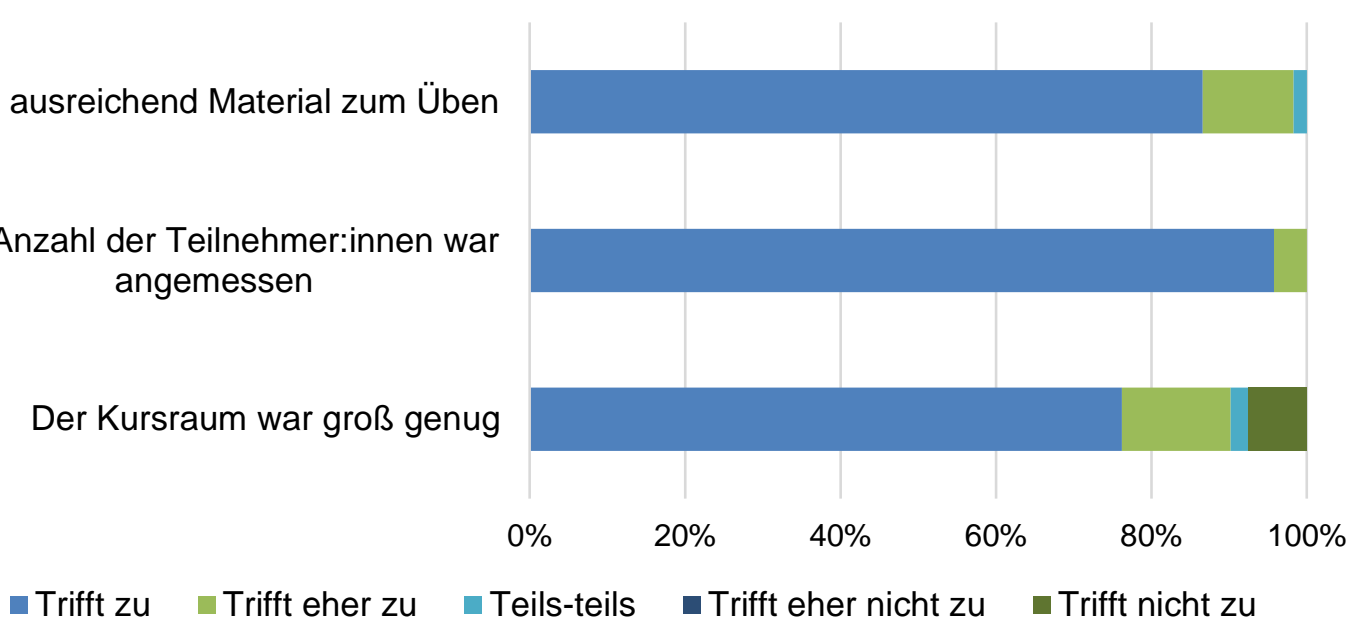

Abbildung 27: Grafische Darstellung der Kursevaluationen zu Räumlichkeiten und Material.

Zusätzlich wurden die Studierenden im Rahmen der Evaluation gefragt, ob innen das im Kurs vorgestellte ABCDE-Schema im Notfall helfen würde. 98,5\% der Studierenden antworteten hier mit „Ja“. 


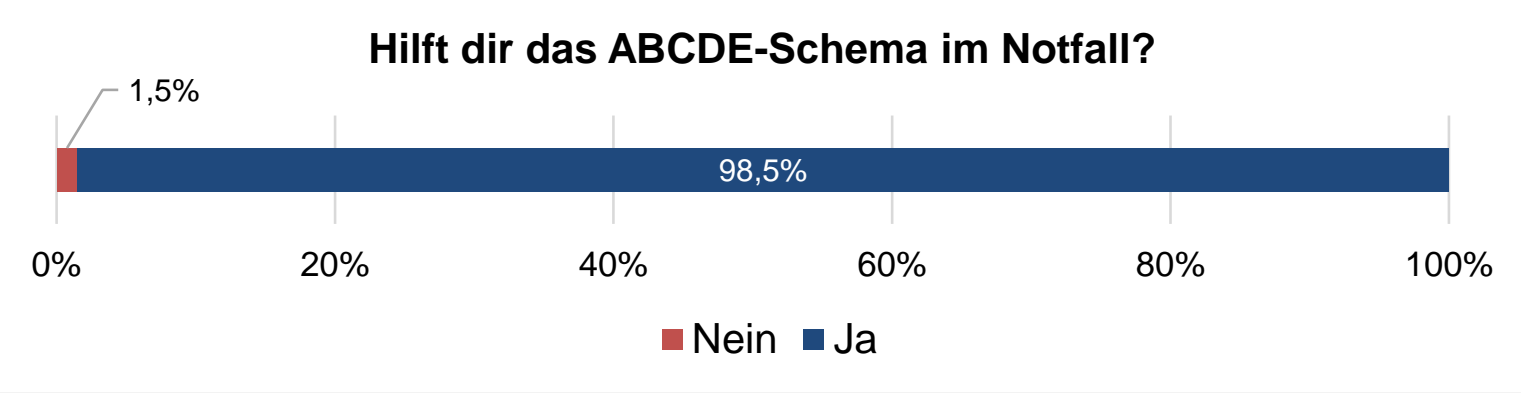

Abbildung 28: Abfrage zum Nutzen des ABCDE-Schemas im Notfall.

Abschließend wurden die Studierenden anhand einer 5-Punkte-Likert-Skala ( $1=$ „trifft zu“/2 = „trifft eher zu“/3 = „teils-teils“/4 = „trifft eher nicht zu“/5 = „trifft nicht zu“) gefragt, ob sie sich nach dem Kurs in der Lage sehen, adäquat Erste Hilfe zu leisten. Der arithmetische Mittelwert lag hier bei 1,43 \pm 0,576, keiner der Studierenden nannte hier die Antwortmöglichkeiten „Trifft eher nicht zu“ oder „Trifft nicht zu“. Die Studierenden schätzten sich wie auch in der Selbsteinschätzung nach dem Kurs durchweg sicher in der Ersten Hilfe ein.

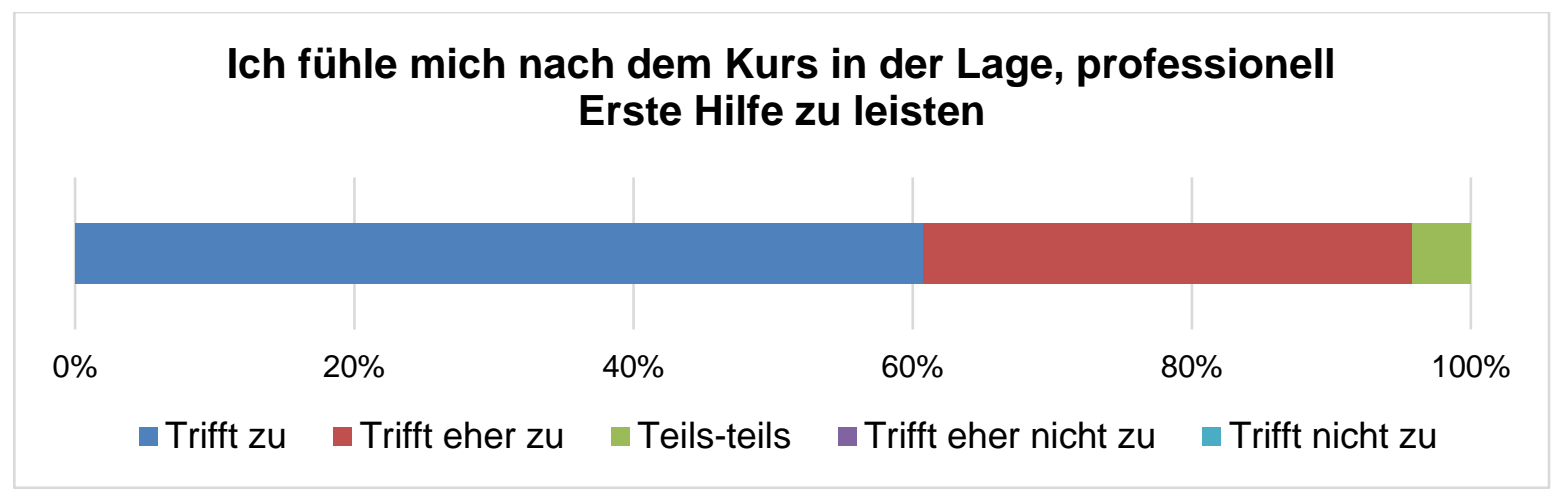

Abbildung 29: Selbsteinschätzung der Erste-Hilfe-Kompetenz nach dem Erste-Hilfe-Kurs.

Am Ende der Kursevaluation hatten die Studierenden die Möglichkeit, sich in einer Freitextantwort zur Frage „Würdest Du den Kurs weiterempfehlen und wenn ja warum, bzw. warum nicht?" zu äußern. Die deutliche Mehrheit der Studierenden würden den Kurs weiterempfehlen. Gründe hierfür waren u.a., weil der Kurs vor allem 
für Medizinstudierende interessant gestaltet und auf den individuellen Lernstand zugeschnitten und auf inn angepasst sei. Auch die eingesetzten klinischen Fälle seien spannend, es gäbe ein durch die motivierten Peer-Dozierende geschaffenes, angenehmes Lernklima und die Studierenden schätzten auch im Freitext noch einmal die vielen praktischen Übungsmöglichkeiten. Auch die Prüfungsstationen wurden von den Studierenden in diesem Zusammenhang positiv erwähnt, da sie ihren Fortschritt direkt nach dem Kurs sehen konnten. 


\section{Diskussion}

In der vorliegenden Studie sollte überprüft werden, welche notfallmedizinischen Basiskompetenzen die Studierenden im vorklinischen Studienabschnitt der GoetheUniversität Frankfurt besitzen. Des Weiteren wurde geprüft, ob ein Erste-Hilfe-Kurs basierend auf dem ABCDE-Schema konzipiert und durchgeführt werden kann und inwiefern dieser die notfallmedizinischen Kompetenzen der Studierenden verbessert. Der Fokus lag auf der Überprüfung, ob sich die methodisch-didaktische Entwicklung des Erste-Hilfe-Kurses bewährte.

\subsection{Zusammenfassung der Ergebnisse vor dem Erste-Hilfe-Kurs}

Es konnte gezeigt werden, dass die notfallmedizinischen Basiskompetenzen der Vorklinikstudierenden der Universität Frankfurt nicht ausreichen, um adäquat mit Notfallsituationen umgehen zu können. Insbesondere die praktischen Maßnahmen der Reanimation waren nicht ausreichend, um eine suffiziente Herzdruckmassage über mindestens 2 Minuten gewährleisten zu können. Bei Durchführung der Stabilen Seitenlage beherrschten weniger als $30 \%$ der Studierenden die Schlüsselmaßnahmen (Kopf überstecken und Mund öffnen). Auch bei der Anwendung eines AED zeigten sich deutliche Defizite in Anwendung, Beachtung der Sicherheit und unverzüglichem Fortführen der Herzdruckmassage.

Zusätzlich demonstrierten die Studierenden in der theoretischen Wissensabfrage im Rahmen des 10 Items MC-Tests deutliche Wissenslücken.

Insgesamt bestätigten die praktische und theoretische Kompetenzüberprüfung das durchweg unsichere Bild der Studierenden in der Selbsteinschätzung vor dem Kurs.

\subsection{Zusammenfassung der Ergebnisse nach dem Erste-Hilfe-Kurs}

Nach dem Kurs zeigten die Studierenden eine in fast allen Punkten signifikante Steigerung ihrer notfallmedizinischen Basiskompetenzen. In den praktischen Stationen bei der Ein-Helfer-Reanimation steigerten sie sich durchschnittlich um 6,8 Punkte 
(max. 18 Punkte), bei der Stabilen Seitenlage um 5,3 Punkte (max. 14 Punkte) und bei der Zwei-Helfer-Reanimation mit Einsetzen eines AED und Anleitung eines Passanten um 5,9 Punkte (max. 14 Punkte). Auch in der Wissensüberprüfung konnten sich die Studierenden in 9 von 10 Antworten signifikant steigern. Dies spiegelt sich auch in einer gesteigerten Selbsteinschätzung nach dem Kurs wider: Während sich vor dem Kurs nur 33\% der Studierenden in der Lage fühlten, Wiederbelebungsmaßnahmen durchzuführen, waren es nach dem Kurs 98\%. Die Kursevaluation am Ende des Kurses war durchweg positiv.

\subsection{Vergleich mit bisheriger Literatur}

Die vorgestellte Studie deckt sich mit den bisherigen Ergebnissen der Literatur: Das Basiswissen und die praktischen Kompetenzen in Erster Hilfe bei Medizinstudierenden reichen nicht aus, um den Erwartungen an sie gerecht zu werden und Notfälle adäquat zu bewältigen. ${ }^{68-74}$ Ein Grund dafür könnte die unstrukturierte und nicht aus-

reichende Ausbildung in der Notfallmedizin in den medizinischen Fakultäten sein. ${ }^{63}$ In dieser sind Vorklinikstudierende und ein auf sie zugeschnittener Erster-Hilfe-Kurs entgegen der Empfehlung von Expert:innen in den meisten Fakultäten nicht integriert. ${ }^{71,75,76}$ Expert:innen fordern bereits in den Schulen eine Ausbildung in kardiopulmonaler Reanimation, um langfristig die Laienreanimationsquoten zu steigern. 2,29,34,53 Es stellt sich die Frage, warum nicht auch bei Medizinstudierenden ein frühzeitiges BLS-Training fest in das Studium integriert wird, wenn es bereits mehrere etablierte Projekte an deutschen Schulen gibt.

Mehrere Studien konnten zeigen, dass auch direkt nach intensivem CPR-Training die geforderten Ziele der Herzdruckmassage nicht erreicht werden konnten. 137,138 Dies zeigte sich auch in dieser Studie: Zwar konnten die praktischen Kompetenzen in den Schlüsselmaßnahmen der Reanimation signifikant gesteigert werden, doch auch nach dem Training erreichten nur $70 \%$ die korrekte Frequenz, 52,5\% die richtige Drucktiefe und nur 66,3\% entlasteten bei der Herzlungenwiederbelebung korrekt. Dies könnte durch längeres und häufigeres Training verbessert werden. 


\subsection{Einfluss individueller Faktoren}

Eine vorherige Teilnahme an Kurskonzepten spielte bei den Ergebnissen der Kompetenzüberprüfung eine geringe Rolle. Die Mehrheit der Studierenden (76,3\%) gab eine vorherige Teilnahme an einem Erste-Hilfe-Kurs an, 13,2\% nahmen bereits an einem Reanimationstraining teil. Bei beiden Angaben lag der Kurs im Mittel 3 Jahre zurück. Es konnte kein Unterschied zwischen diesen Teilnehmenden und dem Rest der Gruppe festgestellt werden. Dies stellten auch andere Studien ausreichend fest, Berden et al. ${ }^{139}$ konnten bspw. keinen Zusammenhang zwischen der Anzahl absolvierter Kurse und der gezeigten Reanimationsleistung von Laien nachweisen.

5,9\% der Studierenden hatten bei Kursteilnahme eine abgeschlossene Berufsausbildung, davon nur ein Teilnehmer im notfallmedizinischen Bereich als Rettungssanitäter. Aus diesem Grund kann davon ausgegangen werden, dass so gut wie keine praktische Vorerfahrungen aufgrund der Berufsausbildung bei den Studierenden vorhanden war. Zusätzlich gaben nur $12 \%$ der Studierenden an, vor Teilnahme des Kurses bereits Erfahrungen in Erster Hilfe gesammelt zu haben, $80 \%$ hatten keine Erfahrungen.

Die Mehrheit der Studierenden (82,9\%) nannte die Notwendigkeit der Vorlage eines Erste-Hilfe-Scheines zur Anmeldung für die erste ärztliche Prüfung als Grund für die Kursteilnahme. Nur 5,3\% der Teilnehmenden gaben eigenes Interesse an und erschienen aus intrinsischer Motivation zum Kurs. Dieses Ergebnis kommt vermutlich durch die Unwissenheit der Studierenden zustande, das eine einmal erlangte ErsteHilfe-Teilnahmebescheinigung nicht noch einmal rezertifiziert werden muss, sofern dies nicht explizit vom Anbieter festgelegt ist. Unabhängig davon erachten es sowohl vor (94\%) als auch nach dem Kurs (95\%) die Studierenden als sinnvoll, einen ErsteHilfe-Kurs als Zulassungsvoraussetzung für das Physikum zu absolvieren, weshalb zu diskutieren ist, ob trotz des genannten Ergebnisses die Studierenden nicht mehr intrinsische Motivation zeigten, als sie an gaben. 
Wichtig ist zu beachten, dass Schulze et al. ${ }^{140}$ zeigen konnten, dass „aus MC-Fragen basierten Prüfungen nicht automatisch abgelesen werden [kann], dass die Studierenden das abgefragte Wissen tatsächlich beherrschen. Der Prüfungserfolg in MC-Fragen basierten Klausuren [ist] zum wesentlichen Teil durch das Fragenformat und den damit verbundenen Entscheidungszwang für eine und nur eine richtige Lösung bedingt ${ }^{140}$.“. Betrachtet man die Ergebnisse der Wissensüberprüfung, wählten die Studierenden auch vor dem Kurs bei 9 von 10 Fragen die richtige Antwort. Ob es sich dabei um fundiertes Wissen handelt, ist aufgrund des Fragenformats schwierig zu beurteilen. Hier fällt auf, dass nach dem Kurs die Studierenden wesentlich häufiger die richtige und seltener die falschen Antwortmöglichkeiten wählten und durch die Absolvierung des Kurses ein Wissenszuwachs erkennbar war.

\subsection{Praktische Implikation}

Der Erste-Hilfe-Kurs für Vorklinikstudierende konnte problemlos in das Angebot des FIneST integriert werden. Aufgrund der hohen Nachfrage der Studierenden werden die Kurse, um die Kleingruppen halten zu können, weiterhin sowohl im Winter- als auch im Sommersemester an mindestens vier Samstagen angeboten. Die vorliegende Arbeit zeigt, dass eine curriculare Einbindung eines solchen Erste-Hilfe-Kurses möglich ist.

\subsubsection{Evaluation des Kurskonzeptes}

Am Ende jeder Veranstaltung findet weiterhin eine ausführliche Online-Evaluation durch die Studierenden statt, sowie eine auf den Ergebnissen der Evaluation basierende Revision der Veranstaltung innerhalb des Tutorenteams unter Betreuung der ärztlichen Dozierenden des FIneST. Nach Lam et al. ${ }^{141}$ ist eine umfassende Kursbewertung, die sowohl quantitative als auch qualitative Methoden verwendet, von Nutzen, um von den Teilnehmenden Informationen darüber zu erhalten, wie eine Optimierung des Kursangebots erreicht werden kann. 
Das durchweg positive Ergebnis der Online-Evaluation wurde zusätzlich durch direktes, mündliches Feedback der Teilnehmenden an die Instruktor:innen am Ende des Kurses unterstützt.

Insgesamt lässt sich aus den Ergebnissen schließen, dass das entwickelte Kurskonzept eines speziell auf Vorklinikstudierende angepassten Erste-Hilfe-Kurses unter der Gliederung des ABCDE-Schemas und mit einem hohen Anteil praktischer Maßnahmen sehr gut von den Studierenden angenommen wird.

Aus der in der Evaluation abgefragten konstruktiven Kritik der Studierenden erfolgte am Ende jedes Semesters bis zum ersten Kurstermin des neuen Semesters eine Verbesserung des Curriculums, der Dozierendenmanuale und den Kurspräsentationen.

\subsection{Limitationen}

Eine Limitation dieser Studie ist die Überprüfung des Langzeiterfolges des ErsteHilfe-Kurses. Bereits in vorangegangenen Studien zeigte sich: „If you teach them, they will learn ${ }^{142 “}$. Somit ist eine Steigerung der notfallmedizinischen Basiskompetenzen nach dem Erste-Hilfe-Kurs zu erwarten. Durch die Arbeit von Cook et al. ${ }^{142}$ wurde hinreichend belegt, dass die Durchführung einer Lehreinheit zu einem Lernerfolg führt. Um jedoch die kurz- und langfristigen notfallmedizinischen Basiskompetenzen der Studierenden zu erfassen, sind Folgestudien durch die Arbeitsgruppe geplant.

Es wurde in der Studie keine Kontrollgruppe mit Teilnehmern, die den entwickelten Kurs nicht besucht haben, gebildet. Die Arbeitsgruppe entschied sich bewusst gegen ein Kontrollgruppen-Studiendesign, um keine Studierenden von der Teilnahme des Kurses auszuschließen.

Zusätzlich handelt es sich bei den drei OSCE-/OSPE-Stationen der praktischen Kompetenzüberprüfung um konstruierte Szenarien, die nach dem ersten Durchgang der Statuserhebung nicht mehr verändert wurden. Demnach hatten die Studieren- 
den nach dem ersten Durchgang Kenntnis über Ablauf und Inhalt der einzelnen Stationen und es kann von einer Gewöhnung an die Situation und Station ausgegangen werden. Aufgrund des Studienprotokolls und um eine Vergleichbarkeit zu gewährleisten, entschied sich die Arbeitsgruppe jedoch für die Beibehaltung der einzelnen Szenarien.

\subsection{Ausblick}

Lam et al. ${ }^{141}$ empfehlen, klinische Fertigkeiten so früh wie möglich in das medizinische Curriculum zu integrieren. Das Training praktischer Fähigkeiten zu Beginn des medizinischen Lehrplans erhöht das Selbstvertrauen der Medizinstudierenden und hatte positive Auswirkungen auf folgende Trainings. ${ }^{143}$

Bereits vor mehr als 20 Jahren zeigten Russ et al. ${ }^{72}$ bereits desaströse BLS-Skills bei Medizinstudierenden und forderten, die Zeiträume zwischen Notfallkursen zu minimieren. „Der Student vergisst das Erlernte zu schnell, da der Bezug zur Praxis fehlt. Refresher-Kurse müssen in das Curriculum aufgenommen werden. ${ }^{72 “}$

Aus diesem Grund sollten frühestmöglich notfallmedizinische Kurse für Studierende angeboten und gleichzeitig ein stetiges Training zur Auffrischung der Fertigkeiten ermöglicht werden. Auch Expert:innen empfehlen ein frühzeitiges BLS-Training im medizinischen Studium sowie z.B. darauf aufbauende Online-Tests im weiteren Verlauf des Studiums. ${ }^{75,144}$ Auch Online-Tutorials vor BLS-Trainings resultierten in signifikant besseren praktischen Maßnahmen der Teilnehmenden. ${ }^{145}$ Der Lerneffekt bei Video-basiertem CPR-Training ist umstritten und aktuell Gegenstand mehrerer Forschungen. ${ }^{144,146-149}$

Bei diesen Beispielen sind vor allem die gute Durchführbarkeit und die RessourcenEinsparung ein wesentlicher Vorteil. Nichtsdestotrotz sollte digitales BLS-Training keine praktischen Kurse ersetzen, ${ }^{150}$ sondern lediglich die Zeitspannen zwischen den Kursen verkürzen. Hands-On-Kurse wie simulationsbasierte Notfallszenarien, Refresher-Kurse und Ergänzungs-Kurse zu den Reanimations- und Erste-Hilfe-Trai- 
nings haben weiterhin die größte Auswirkung auf die Fähigkeiten der Teilnehmenden. ${ }^{151}$ Insbesondere Refresher-Kurse sind in verschiedenen zeitlichen Abständen von 6 bis 24 Monaten empfohlen. ${ }^{152-156}$

Basierend auf diesem Wissen sollte die notfallmedizinische Ausbildung von Medizinstudierenden überarbeitet und angepasst werden. Beginnend mit einem ins Studium integrierten Erste-Hilfe-Kurs für Vorklinikstudierende und regelmäßigen Refresher-Kursen in möglichst kurzen Zeitabstanden können die Studierenden auf den klinischen Notfallkurs und die wachsenden Ansprüche im klinischen Altag vorbereitet werden. Online-Ressourcen wie Video-basiertes CPR-Training könnten, um Kosten und Personal zu sparen, in den Lehrplan integriert werden und so für eine stringente notfallmedizinische Ausbildung von Medizinstudierenden sorgen.

\subsection{Schlussfolgerung}

Schon seit über 30 Jahren wird eine strukturierte notfallmedizinische Ausbildung von Medizinstudierenden gefordert, da immer wieder gezeigt werden konnte, dass hier deutliche Mängel vorliegen. Die vorliegende Arbeit konnte erneut aufzeigen, dass Vorklinikstudierende weiterhin erhebliche Defizite in notfallmedizinischen Basiskompetenzen vorweisen. Trotz vorheriger Teilnahme an gängigen Erste-Hilfe-Kursen sind die praktischen und theoretischen Kenntnisse der Studierenden ungenügend. Dies spiegelt auch die durchweg unsichere Selbsteinschätzung der Studierenden wider - im Notfall wären sie mit einer Notfallsituation tendenziell eher überfordert statt kompetent unterstützend und helfend zu agieren. Die Ausgliederung der ErsteHilfe-Teilnahmebescheinigung an andere Anbieter und die Tatsache, dass ein einmal absolvierter Erste-Hilfe-Schein nicht rezertifiziert werden muss, begünstigen, dass Studierende keine adäquaten notfallmedizinischen Basiskompetenzen vorweisen können. In der Studie konnte herausgearbeitet werden, dass ein speziell für Vorklinikstudierende konzipierter Erste-Hilfe-Kurs, am ABCDE-Schema orientiert, mit einem hohen praktischen Anteil und der an das bereits gelernte Wissen anknüpft, zu signifikant besseren notfallmedizinischen Basiskompetenzen führt. Der Kurs 
sollte den universitären, medizinischen und curricularen Ansprüchen gerecht werden und für alle Studierenden verpflichtend und kostenlos sein, um eine breitflächige adäquate Ausbildung auf höchstem Niveau zu gewährleisten. Eine weitere Erkenntnis ist, dass der Erste-Hilfe-Kurs nach den beschriebenen Vorbereitungen fest in das Angebot des FIneST integriert werden konnte. Dadurch wurde das notfallmedizinische Angebot im Querschnittsbereich 8 der Goethe-Universität Frankfurt auch um einen Kurs für Vorklinikstudierende erweitert.

Die dargestellten Ergebnisse rechtfertigen die Aussage, dass die Einführung eines curricular fest integrierten Erste-Hilfe-Kurses in die vorklinischen Studiumsinhalte sinnhaft und zwingend notwendig ist. Die Implementierung eines solchen Kurses sollte daher ein Ziel innerhalb der Umsetzung des Masterplan 2020 in den nächsten Jahren darstellen. 


\section{Literaturverzeichnis}

1. Sefrin P. Geschichte der Notfallmedizin in Deutschland - unter besonderer Berücksichtigung des Notarztdienstes. Anasthesiol Intensivmed Notfallmed Schmerzther. 2003;38(10):623-629.

2. Burghofer K, Köhler M, Stolpe E, Lackner C. Erste-Hilfe-Maßnahmen bei Notfällen. Notfall+ Rettungsmedizin. 2008;11(2):127-136.

3. Donner-Banzhoff N, Schuster C, Hofmann S, Baum E. Notfallversorgung durch Ersthelfer. Anasthesiol Intensivmed Notfallmed Schmerzther. 1999;34(03):140-146.

4. Lick RF, Schläfer H. Unfallrettung : Medizin u. Technik. Stuttgart, New York; 1973.

5. Deutsche Gesetzliche Unfallversicherung. 2015. https://www.dguv.de/medien/fberstehilfe/de/documents/revision.pdf. Accessed April 03, 2020.

6. Winkler B, Henssler J, Piepho T, Georgieff M, Muth C-M, Dinse-Lambracht A. Laienreanimation nach kürzlich durchgeführtem Erste-Hilfe-Kurs. Notfall+ Rettungsmedizin. 2014;17(8):684-689.

7. Beckers S. Organisation und Struktur. In: Brokmann J, Rossaint R, eds. Repetitorium Notfallmedizin: Zur Vorbereitung auf die Prüfung 》Notfallmedizin《. Berlin, Heidelberg: Springer Berlin Heidelberg; 2008:3-40.

8. (BGW) BfGuW. DGUV Vorschrift 1 „Grundsätze der Prävention“. DGUV Vorschrift. 1. Oktober 2014.

9. BAGEH. Unsere Mitgliedsorganisationen. 2020; https://www.bageh.de/wer-wirsind/mitgliedsorganisationen. Accessed April 04, 2020.

10. BAGEH. Was wir tun. 2020; https://www.bageh.de/wer-wir-sind/was-wir-tun. Accessed April 03, 2020.

11. Zideman DA, De Buck EDJ, Singletary EM, et al. Erste Hilfe. Notfall + Rettungsmedizin. 2015;18(8):1003-1015.

12. Ahnefeld FW. Die Rettungskette: eine Idee wurde Wirklichkeit. Notfall \& Rettungsmedizin. 2003;6(7):520-525.

13. Deutsches

Reanimationsregister. https://www.reanimationsregister.de/docman/oeffentliche-jahresberichte/cardiacarrest-center/174-cac-oeffentlicher-jahresbericht-2019/file.html. Accessed April 05, 2020. 
14. Bundesamt für Statistik. Todesursachen nach Krankheitsarten 2018. https://www.destatis.de/DE/Themen/GesellschaftUmwelt/Gesundheit/Todesursachen/_inhalt.html. Accessed August 25, 2019.

15. Christ M, von Auenmüller KI, Grett M, Dierschke W, Trappe HJ. Wer beobachtet in Deutschland den außerklinischen Herzstillstand? Dtsch Med Wochenschr. 2014;139(44):2225-2230.

16. Wnent J, Bohn A, Seewald S, et al. [Bystander resuscitation: the impact of first aid on survival]. Anasthesiol Intensivmed Notfallmed Schmerzther. 2013;48(9):562-565.

17. Deppe M, Uzun DD, Bläser D. Aktualisierung der ERC-Leitlinien 2018. Notfmed up2date. 2019;14(03):251-262.

18. GRC. Reanimation 2015. Leitlinien kompakt. 2015;7.

19. Hasselqvist-Ax I, Riva G, Herlitz J, et al. Early Cardiopulmonary Resuscitation in Outof-Hospital Cardiac Arrest. N Engl J Med. 2015;372(24):2307-2315.

20. Wissenberg M, Lippert FK, Folke F, et al. Association of National Initiatives to Improve Cardiac Arrest Management With Rates of Bystander Intervention and Patient Survival After Out-of-Hospital Cardiac Arrest. JAMA. 2013;310(13):13771384.

21. de Waha S, Desch S, Eitel I, Gräsner JT, Jakisch B, Thiele H. Prognostische Bedeutung der Laienreanimation bei extrahospitalem Herz-Kreislauf-Stillstand. Medizinische Klinik - Intensivmedizin und Notfallmedizin. 2017;112(8):737-740.

22. Gräsner J-T, Wnent J, Gräsner I, Seewald S, Fischer M, Jantzen T. Einfluss der Basisreanimationsmaßnahmen durch Laien auf das Überleben nach plötzlichem Herztod. Notfall+ Rettungsmedizin. 2012;15(7):593-599.

23. Groß R, Böttiger BW, Thaiss HM. Laienreanimation in Deutschland: Das Nationale Aktionsbündnis Wiederbelebung (NAWIB). Notfall + Rettungsmedizin. 2019;22(8):715-722.

24. Rajan S, Wissenberg M, Folke F, et al. Association of bystander cardiopulmonary resuscitation and survival according to ambulance response times after out-ofhospital cardiac arrest. Circulation. 2016;134(25):2095-2104.

25. Grasner JT, Wnent J, Herlitz J, et al. Survival after out-of-hospital cardiac arrest in Europe - Results of the EuReCa TWO study. Resuscitation. 2020;148:218-226.

26. Piepho T, Resch N, Heid F, Werner C, Noppens RR. Lay basic life support: the current situation in a medium-sized German town. Emerg Med J. 2011;28(9):786789. 
27. Parnell MM, Larsen PD. Poor quality teaching in lay person CPR courses. Resuscitation. 2007;73(2):271-278.

28. Breckwoldt J, Lingemann C, Wagner P. [Resuscitation training for lay persons in first aid courses: Transfer of knowledge, skills and attitude]. Anaesthesist. 2016;65(1):2229.

29. Bohn A, Seewald S, Wnent J. Reanimation - Basismaßnahmen bei Erwachsenen und Anwendung automatischer externer Defibrillatoren. Anasthesiol Intensivmed Notfallmed Schmerzther. 2016;51(03):178-186.

30. Tang HM, Wu X, Jin Y, et al. Shorter training intervals increase high school students' awareness of cardiopulmonary resuscitation: a questionnaire study. J Int Med Res. 2020;48(1):300060519897692.

31. Lee JH, Cho Y, Kang KH, Cho GC, Song KJ, Lee CH. The Effect of the Duration of Basic Life Support Training on the Learners' Cardiopulmonary and Automated External Defibrillator Skills. Biomed Res Int. 2016;2016:2420568.

32. Lund-Kordahl I, Mathiassen M, Melau J, Olasveengen TM, Sunde K, Fredriksen K. Relationship between level of CPR training, self-reported skills, and actual manikin test performance-an observational study. Int J Emerg Med. 2019;12(1):2.

33. Hsieh MJ, Chiang WC, Jan CF, Lin HY, Yang CW, Ma MH. The effect of different retraining intervals on the skill performance of cardiopulmonary resuscitation in laypeople-A three-armed randomized control study. Resuscitation. 2018;128:151157.

34. Wiese C, Wilke H, Bahr J, Adler M, Graf B. Kurs „Lebensrettende Sofortmaßnahmen“. Notfall+ Rettungsmedizin. 2008;11(7):482-486.

35. Wiese CHR BU, Bahr J, Graf BM. Durchführung von CPR-Basismaßnahmen durch Laienhelfer nach dem Kurs "Lebensrettende Sofortmaßnahmen“. Notfall Rettungsmed. 2007:10:221-228.

36. Christ $M$, van Bracht $M$, Prull MW. Einflüsse medizinischer Aufklärungsarbeit auf Erste-Hilfe- und AED-Kenntnisse in der Allgemeinbevölkerung. Dtsch Med Wochenschr. 2012;137:2251-2255.

37. Lackner CKK, K.G.; S.Rothenberger, S.; Ruppert, M. AED-Anwenderperformanz von Laien- und Ersthelfern. Notfall \& Rettungsmedizin. 2001:4:572-584.

38. Bunch TJ, White RD, Gersh BJ, et al. Long-Term Outcomes of Out-of-Hospital Cardiac Arrest after Successful Early Defibrillation. $N$ Engl $J$ Med. 2003;348(26):2626-2633. 
39. White RD, Hankins DG, Bugliosi TF. Seven years' experience with early defibrillation by police and paramedics in an emergency medical services system. Resuscitation. 1998;39(3):145-151.

40. Rho RW, Page RL. The automated external defibrillator. $J$ Cardiovasc Electrophysiol. 2007;18(8):896-899.

41. Ramaswamy K, Page RL. The automated external defibrillator: critical link in the chain of survival. Annu Rev Med. 2003;54(1):235-243.

42. Arntz HR. Frühdefibrillation in Deutschland - Entwicklung und Erfahrungen im arztbegleiteten Rettungsdienst. Notfall \& Rettungsmedizin. 2004:7:12-17.

43. Tries R. Frühdefibrillation aus juristischer Sicht. Rettungsdienst. 1999;22:18-25.

44. Davies CS, Colquhoun M, Boyle R, Chamberlain D. A national programme for onsite defibrillation by lay people in selected high risk areas: initial results. Heart. 2005;91(10):1299-1302.

45. Valenzuela TD, Roe DJ, Nichol G, Clark LL, Spaite DW, Hardman RG. Outcomes of rapid defibrillation by security officers after cardiac arrest in casinos. $N$ Engl J Med. 2000;343(17):1206-1209.

46. Beckers SK, Fries M, Bickenbach J, et al. Retention of skills in medical students following minimal theoretical instructions on semi and fully automated external defibrillators. Resuscitation. 2007;72(3):444-450.

47. Perkins GD, Handley AJ, Koster RW, et al. European Resuscitation Council Guidelines for Resuscitation 2015: Section 2. Adult basic life support and automated external defibrillation. Resuscitation. 2015;95:81-99.

48. Lawson L, March J. Automated external defibrillation by very young, untrained childen. Prehosp Emerg Care. 2002;6(3):295-298.

49. Lackner CK, Wendt M, Ahnefeld FW, Koch B. Von der Rettungskette zum akutmedizinischen Netzwerk. Notfall + Rettungsmedizin. 2009;12(1):25-31.

50. Bundesarbeitsgemeinschaft Erste $\mathrm{H}$. Aus- und Fortbildung von Ersthelfern in der Frühdefibrillation. Notfall \& Rettungsmedizin. 2004;7(1):34-35.

51. Zinckernagel L, Hansen CM, Rod MH, Folke F, Torp-Pedersen C, Tjørnhøj-Thomsen T. A qualitative study to identify barriers to deployment and student training in the use of automated external defibrillators in schools. BMC Emerg Med. 2016;17(1):3.

52. Wurmb T, Vollmer T, Sefrin P, et al. Monitoring of in-hospital cardiac arrest events with the focus on Automated External Defibrillators--a retrospective observational study. Scand J Trauma Resusc Emerg Med. 2015;23:87. 
53. Lukas R-P, Bohn A, Möllhoff T, Aken HKV. Laienreanimation - Reanimation als Schulfach: „Was Hänschen nicht lernt ..... Anasthesiol Intensivmed Notfallmed Schmerzther. 2013;48(09):552-557.

54. Aaberg AM, Larsen CE, Rasmussen BS, Hansen CM, Larsen JM. Basic life support knowledge, self-reported skills and fears in Danish high school students and effect of a single 45-min training session run by junior doctors; a prospective cohort study. Scand J Trauma Resusc Emerg Med. 2014;22:24.

55. Jorge-Soto C, Abilleira-Gonzalez M, Otero-Agra M, et al. Schoolteachers as candidates to be basic life support trainers: A simulation trial. Cardiol J. 2019;26(5):536-542.

56. Meissner TM, Kloppe C, Hanefeld C. Basic life support skills of high school students before and after cardiopulmonary resuscitation training: a longitudinal investigation. Scand J Trauma Resusc Emerg Med. 2012;20:31.

57. Gräsner JT, Geldner G, Werner C, et al. Optimierung der Reanimationsversorgung in Deutschland. Notfall + Rettungsmedizin. 2014;17(4):314-316.

58. Breckwoldt J, Kreimeier U. Ausbildung von Schülern zu Ersthelfern bei der Reanimation. Notfall+ Rettungsmedizin. 2013;16(5):356-360.

59. Plant N, Taylor K. How best to teach CPR to schoolchildren: A systematic review. Resuscitation. 2013;84(4):415-421.

60. Tanaka S, Hara T, Tsukigase K, et al. A pilot study of Practice While Watch based 50 min school quality cardiopulmonary resuscitation classroom training: a cluster randomized control trial. Acute Med Surg. 2020;7(1):e455.

61. Burdick WP, Jouriles NJ, D'Onofrio G, et al. Emergency Medicine in Undergraduate Education. Acad Emerg Med. 1998;5(11):1105-1110.

62. Verbraucherschutz BfJu. ÄApprO 2002. 27.06.2002; https://www.gesetze-iminternet.de/_appro_2002/BJNR240500002.html. Accessed April 04, 2018.

63. Beckers SK, Timmermann A, Muller MP, Angstwurm M, Walcher F. Undergraduate medical education in emergency medical care: a nationwide survey at German medical schools. BMC Emerg Med. 2009;9:7.

64. Pfeilschifter J. Studienordnung für den Studiengang Medizin an der GoetheUniversität Frankfurt am Main. In: Main G-UFa, ed. Frankfurt: Präsidentin der Goethe-Universität Frankfurt am Main; 2019.

65. Pfeilschifter J. Studienordnung für den Studiengang Medizin an der GoetheUniversität Frankfurt am Main. In: Main G-UFa, ed. Frankfurt: Präsidentin der Goethe-Universität Frankfurt; 2014. 
66. Beckers S, Fries M, Bickenbach J, et al. Evaluation of a new approach to implement structured, evidence-based emergency medical care in undergraduate medical education in Germany. Resuscitation. 2005;65(3):345-356.

67. Iblher P, Iblher H, Wolff RF, Harbs H, Hüppe M, Eichler W. Neue Wege in der Notfallausbildung von Medizinstudierenden. Der Anaesthesist. 2009;58(4):362.

68. Ammirati C, Gignon M, Amsallem C, et al. Use of an automated external defibrillator: a prospective observational study of first-year medical students. Resuscitation. 2011;82(2):195-198.

69. Grześkowiak M. The effects of teaching basic cardiopulmonary resuscitation-A comparison between first and sixth year medical students. Resuscitation. 2006;68(3):391-397.

70. Korber MI, Kohler T, Weiss V, Pfister R, Michels G. Quality of Basic Life Support - A Comparison between Medical Students and Paramedics. J Clin Diagn Res. 2016;10(7):OC33-37.

71. Lesnik D, Lesnik B, Golub J, Krizmaric M, Mally S, Grmec S. Impact of additional module training on the level of basic life support knowledge of first year students at the University of Maribor. Int J Emerg Med. 2011;4:16.

72. Russ W, Kanz KG, Biberthaler $P$, et al. Theoretische Kenntnisse und praktische Fertigkeiten in der Basisreanimation. Notfall \& Rettungsmedizin. 1998;1(4):214-222.

73. Willmore RD, Veljanoski D, Ozdes F, et al. Do medical students studying in the United Kingdom have an adequate factual knowledge of basic life support? World $J$ Emerg Med. 2019;10(2):75-80.

74. Remmen R. Unsatisfactory basic skills performance by students in traditional medical curricula. Med Teach. 1998;20(6):579-582.

75. Lami M, Nair P, Gadhvi K. Improving basic life support training for medical students. Adv Med Educ Pract. 2016;7:241-242.

76. Tipa RO, Bobirnac G. Importance of basic life support training for first and second year medical students - a personal statement. J Med Life. 2010;3(4):465-467.

77. Seidman PA, Maloney LM, Olvet DM, Chandran L. Preclinical Simulation Training of Medical Students Results in Better Procedural Skills Performance in End of the Year Three Objective Structured Clinical Evaluation Assessments. Medical Science Educator. 2017;27(1):89-96.

78. Störmann $\mathrm{S}$, Stankiewicz $\mathrm{M}$, Raes $\mathrm{P}$, et al. How well do final year undergraduate medical students master practical clinical skills? GMS journal for medical education. 2016;33(4). 
79. Dafsari HS, Herzig S, Matthes J. A multi-centre student survey on weighing disciplines in medical curricula-a pilot study. GMS journal for medical education. 2017;34(2).

80. Remmen R, Scherpbier A, Van Der Vleuten C, et al. Effectiveness of basic clinical skills training programmes: a cross-sectional comparison of four medical schools. Med Educ. 2001;35(2):121-128.

81. Seale J, Knoetze M, Phung A, Prior D, Butchers C. Commencing Technical Clinical Skills Training in the Early Stages of Medical Education: Exploring Student Views. Medical Science Educator. 2019;29(1):173-179.

82. Linßen SK. Implementierung eines strukturierten schriftlichen Feedbacks in eine bestehende OSCE-Prüfung [dissertation]. Frankfurt am Main: Johann Wolfgang Goethe-Universität Frankfurt; 2020.

83. Häske D, Gliwitzky B, Münzberg M. Notfallmedizin - Standardisierte Kursformate. Lege artis. 2015;5(02):110-116.

84. Fritzsche K, Jantzen T, Rüsseler M, Müller MP. Notfälle im Krankenhaus Ausbildungskonzepte für innerklinische Notfallsituationen. Anasthesiol Intensivmed Notfallmed Schmerzther. 2013;48(06):406-413.

85. Gliwitzky B, Prückner S, Röhrenbeck C, et al. Regelmäßiges Training verbessert die Qualität der Reanimation. Notfall + Rettungsmedizin. 2014;17(4):336-337.

86. Münzberg M, Mahlke L, Bouillon B, Paffrath T, Matthes G, Wölfl CG. Sechs Jahre Advanced Trauma Life Support (ATLS) in Deutschland. Der Unfallchirurg. 2010;113(7):561-567.

87. Häske D, Gliwitzky B, Semmel T, et al. Advanced Medical Life Support (AMLS). Notfall+ Rettungsmedizin. 2013;16(8):611-616.

88. Brokmann J, Beckers S, Skorning M, Wölfl C, Sopka S, Rossaint R. Evidenzbasierte Medizin in der notfallmedizinischen Fort-und Weiterbildung. Notfall+ Rettungsmedizin. 2009;12(5):360-365.

89. Lackner CK, Reith M, Kerkmann R, Peter K. Leitlinien in der Notfallmedizin. Notfall \& Rettungsmedizin. 1998;1(5):314-323.

90. Sefrin P. Leitlinien und Algorithmen - Hilfen oder Grenzen der Notfallmedizin? Intensivmedizin und Notfallmedizin. 2002;39(7):617-621.

91. Thim T, Krarup NH, Grove EL, Rohde CV, Lofgren B. Initial assessment and treatment with the Airway, Breathing, Circulation, Disability, Exposure (ABCDE) approach. Int J Gen Med. 2012;5:117-121. 
92. Gräff I, Lenkeit S. Innerklinische Akutversorgung von Notfallpatienten. Medizinische Klinik - Intensivmedizin und Notfallmedizin. 2014;109(7):479-484.

93. Ziegenfuß T. Notfallmedizin. In. Springer-Lehrbuch. 7th ed. 2017 ed. Berlin, Heidelberg 2017.

94. Jakisch B, Gräsner J-T, Seewald S, Renzing N, Wnent J. Aktuelle Behandlungskonzepte des „traumatischen Herz-Kreislauf-Stillstands “. Der Anaesthesist. 2019;68(3):132-142.

95. Kern DE, Thomas PA, Howard DM, Bass EB. Curriculum Development for Medical Education - A Six Step Approach. Baltimore: The John Hopkins University Press; 1998.

96. Soar J, Monsieurs K, Ballance J, et al. Unterrichtsprinzipien zur Wiederbelebung. Notfall + Rettungsmedizin. 2010;13(7):723-736.

97. Aarnio M, Nieminen J, Pyörälä E, Lindblom-Ylänne S. Motivating medical students to learn teamwork skills. Med Teach. 2010;32(4):e199-e204.

98. Knowles MS. The adult learner: a neglected species. Houston, Texas: Gulf Publishing Company; 1990.

99. Vaughn L, Baker R. Teaching in the medical setting: balancing teaching styles, learning styles and teaching methods. Medical teacher. 2001;23(6):610-612.

100. Hutchinson L. Educational environment. BMJ. 2003;326(7393):810-812.

101. Ruesseler M, Obertacke U. Teaching in daily clinical practice: how to teach in a clinical setting. Eur J Trauma Emerg Surg. 2011;37:313-316.

102. Ahlemann F. Strategisches Projektmanagement : Praxisleitfaden, Fallstudien und Trends. In. 1st ed. 2013 ed. Berlin, Heidelberg: Eckl, Christoph; 2013.

103. Kirkbright S, Finn J, Tohira H, Bremner A, Jacobs I, Celenza A. Audiovisual feedback device use by health care professionals during CPR: A systematic review and metaanalysis of randomised and non-randomised trials. Resuscitation. 2014;85(4):460471.

104. Spooner BB, Fallaha JF, Kocierz L, Smith CM, Smith SCL, Perkins GD. An evaluation of objective feedback in basic life support (BLS) training. Resuscitation. 2007;73(3):417-424.

105. Wagner M, Bibl K, Hrdliczka E, et al. Effects of Feedback on Chest Compression Quality: A Randomized Simulation Study. Pediatrics. 2019;143(2).

106. NKLM. Kapitel $17 \quad$ Notfallmaßnahmen. 2015 http://www.nklm.de/kataloge/nklm/lernziel/uebersicht\#. Accessed April 10, 2020. 
107. Ali A, Evans P. Multi-resource peer assisted learning in postgraduate setting: a pilot study. Journal of the College of Physicians and Surgeons Pakistan. 2013;23(4):251256.

108. Herrmann-Werner A, Gramer R, Erschens R, et al. Peer-assisted learning (PAL) in undergraduate medical education: An overview. Zeitschrift für Evidenz, Fortbildung und Qualität im Gesundheitswesen. 2017;121:74-81.

109. Blohm M, Lauter J, Branchereau S, et al. " Peer-Assisted Learning"(PAL) in the Skills-Lab-an inventory at the medical faculties of the Federal Republic of Germany. GMS Zeitschrift Für Medizinische Ausbildung. 2015;32(1).

110. Burgess $A$, McGregor D, Mellis $C$. Medical students as peer tutors: a systematic review. BMC Med Educ. 2014;14(1):115.

111. Dîrzu DS, Hagău N, Boț T, Fărcaș L, Copotoiu SM. Training in cardiopulmonary resuscitation provided by medical students, residents and specialists: A noninferiority trial. Hong Kong Journal of Emergency Medicine. 2018;25(1):20-26.

112. Toner P, Connolly M, Laverty L, McGrath P, Connolly D, McCluskey DR. Teaching basic life support to school children using medical students and teachers in a 'peertraining' model-Results of the 'ABC for life' programme. Resuscitation. 2007;75(1):169-175.

113. Breckwoldt J, Beetz D, Schnitzer L, Waskow C, Arntz H-R, Weimann J. Medical students teaching basic life support to school children as a required element of medical education: a randomised controlled study comparing three different approaches to fifth year medical training in emergency medicine. Resuscitation. 2007;74(1):158-165.

114. Fujiwara $T$, Nishimura $M$, Honda $R$, et al. Comparison of peer-led versus professional-led training in basic life support for medical students. Adv Med Educ Pract. 2011;2:187-191.

115. Beck S, Issleib M, Daubmann A, Zollner C. Peer education for BLS-training in schools? Results of a randomized-controlled, noninferiority trial. Resuscitation. 2015;94:85-90.

116. Abbas F, Sawaf B, Hanafi I, et al. Peers versus professional training of basic life support in Syria: a randomized controlled trial. BMC Med Educ. 2018;18(1):142.

117. Harvey PR, Higenbottam CV, Owen A, Hulme J, Bion JF. Peer-led training and assessment in basic life support for healthcare students: synthesis of literature review and fifteen years practical experience. Resuscitation. 2012;83(7):894-899.

118. Hughes TC, Jiwaji Z, Lally K, et al. Advanced Cardiac Resuscitation Evaluation (ACRE): a randomised single-blind controlled trial of peer-led vs. expert-led advanced resuscitation training. Scand J Trauma Resusc Emerg Med. 2010;18:3. 
119. Jackson TA, Evans DJR. Can medical students teach? A near-peer-led teaching program for year 1 students. Adv Physiol Educ. 2012;36(3):192-196.

120. Naeger DM, Conrad M, Nguyen J, Kohi MP, Webb EM. Students teaching students: evaluation of a "near-peer" teaching experience. Acad Radiol. 2013;20(9):11771182.

121. Nelson AJ, Nelson SV, Linn AMJ, Raw LE, Kildea HB, Tonkin AL. Tomorrow's educators ... today? Implementing near-peer teaching for medical students. Med Teach. 2013;35(2):156-159.

122. Furmedge DS, Iwata K, Gill D. Peer-assisted learning-Beyond teaching: How can medical students contribute to the undergraduate curriculum? Med Teach. 2014;36(9):812-817.

123. Yu T-C, Wilson NC, Singh PP, Lemanu DP, Hawken SJ, Hill AG. Medical studentsas-teachers: a systematic review of peer-assisted teaching during medical school. Advances in medical education and practice. 2011;2:157-172.

124. Kanz KG, Russ W, Eitel F, Deiler S, Biberthaler P, Mutschler W. Implementierung eines Qualitätsmanagementsystems für die Unterrichtsdurchführung des Notfallkurses. Gesundheitswesen. 2000;62(06):347-354.

125. Mahling M, Munch A, Schenk S, et al. Basic life support is effectively taught in groups of three, five and eight medical students: a prospective, randomized study. BMC Med Educ. 2014;14:185.

126. Cho Y, Je S, Yoon YS, et al. The effect of peer-group size on the delivery of feedback in basic life support refresher training: a cluster randomized controlled trial. BMC Med Educ. 2016;16:167.

127. Kromrey H. Studierendenbefragungen als Evaluation der. Hochschul-Ranking: zur Qualitätsbewertung von Studium und Lehre. 2001:11.

128. Schuwirth LW, van der Vleuten CP. Challenges for educationalists. BMJ. 2006;333(7567):544-546.

129. Oppitz M, Schriek G, Busch C, Shiozawa T, Drews U. Offene Fragen vs. MultipleChoice-Fragen im 1. Abschnitt des Medizinstudiums: Untersuchung am Beispiel von Topographischer Anatomie. GMS Z Med Ausbild. 2007;24.

130. Möltner A, Schellberg D, Jünger J. Grundlegende quantitative Analysen medizinischer Prüfungen. GMS Z Med Ausbild. 2006;23(3):2006-2023.

131. Öchsner W, Böckers A. Ärztliche Fachzeitschriften als implizite Vorbilder für gute Multiple-Choice-Fragen - in welchem Maß erfüllen die Tests der continous medical education inzwischen formale Gütekriterien? Zeitschrift für Evidenz, Fortbildung und Qualität im Gesundheitswesen. 2013;107(7):468-474. 
132. Müller S, Dahmen U, Settmacher U. Objective Structured Clinical Examination (OSCE) an Medizinischen Fakultäten in Deutschland - eine Bestandsaufnahme. Gesundheitswesen. 2018;80(12):1099-1103.

133. Kruppa E, Jünger J, Nikendei C. Einsatz innovativer Lern- und Prüfungsmethoden an den Medizinischen Fakultäten der Bundesrepublik Deutschland - Eine aktuelle Bestandsaufnahme. Dtsch Med Wochenschr. 2009;134(08):371-372.

134. Jünger J, Schellberg $\mathrm{D}$, Nikendei $\mathrm{C}$. Subjektive Kompetenzeinschätzung von Studierenden und ihre Leistung im OSCE. GMS Z Med Ausbild. 2006;23(3):20062023.

135. Iblher P, Zupanic M, Härtel C, et al. Im Notfall bereit? OSCE in der studentischen Notfallausbildung. 2011.

136. Schwill S, Fahrbach-Veeser J, Moeltner A, et al. Peers as OSCE assessors for junior medical students - a review of routine use: a mixed methods study. BMC Med Educ. 2020;20(1):17.

137. Berger $\mathrm{C}$, Brinkrolf $\mathrm{P}$, Ertmer $\mathrm{C}$, et al. Combination of problem-based learning with high-fidelity simulation in CPR training improves short and long-term CPR skills: a randomised single blinded trial. BMC Med Educ. 2019;19(1):180.

138. de Ruijter PA, Biersteker HA, Biert J, van Goor H, Tan EC. Retention of first aid and basic life support skills in undergraduate medical students. Med Educ Online. 2014;19:24841.

139. Berden HJ, Bierens JJ, Willems FF, Hendrick JM, Pijls NH, Knape JT. Resuscitation skills of lay public after recent training. Ann Emerg Med. 1994;23(5):1003-1008.

140. Schulze J, Drolshagen S, Nürnberger F, Ochsendorf F, Schäfer V, Brandt C. Einfluss des Fragenformates in Multiple-choice-Prüfungen auf die Antwortwahrscheinlichkeit: eine Untersuchung am Beispiel mikrobiologischer Fragen. Universitätsbibliothek Johann Christian Senckenberg; 2006.

141. Lam TP, Irwin M, Chow LWC, Chan P. Early introduction of clinical skills teaching in a medical curriculum - factors affecting students' learning. Med Educ. 2002;36(3):233-240 .

142. Cook DA. If you teach them, they will learn: why medical education needs comparative effectiveness research. In: Springer; 2012.

143. Liddell MJ, Davidson SK, Taub H, Whitecross LE. Evaluation of procedural skills training in an undergraduate curriculum. Med Educ. 2002;36(11):1035-1041.

144. Arora V, Bala M, Chawla S. Impact of additional short session of video training on performance of basic life support skills in 2nd-Year medical students. Indian journal 
of critical care medicine: peer-reviewed, official publication of Indian Society of Critical Care Medicine. 2018;22(7):498.

145. Bylow $\mathrm{H}$, Karlsson $\mathrm{T}$, Lepp M, Claesson A, Lindqvist J, Herlitz J. Effectiveness of web-based education in addition to basic life support learning activities: A cluster randomised controlled trial. PLoS One. 2019;14(7).

146. Beskind DL, Stolz U, Thiede R, et al. Viewing a brief chest-compression-only CPR video improves bystander CPR performance and responsiveness in high school students: A cluster randomized trial. Resuscitation. 2016;104:28-33.

147. Braslow A, Brennan RT, Newman MM, Bircher NG, Batcheller AM, Kaye W. CPR training without an instructor: development and evaluation of a video selfinstructional system for effective performance of cardiopulmonary resuscitation. Resuscitation. 1997;34(3):207-220.

148. Godfred R, Huszti E, Fly D, Nichol G. A randomized trial of video self-instruction in cardiopulmonary resuscitation for lay persons. Scand J Trauma Resusc Emerg Med. 2013;21(1):36.

149. Todd KH, Braslow A, Brennan RT, et al. Randomized, Controlled Trial of Video SelfInstruction Versus Traditional CPR Training. Ann Emerg Med. 1998;31(3):364-369.

150. Lau Y, Nyoe RSS, Wong SN, Ab Hamid ZB, Leong BS-H, Lau ST. Effectiveness of digital resuscitation training in improving knowledge and skills: A systematic review and meta-analysis of randomised controlled trials. Resuscitation. 2018;131:14-23.

151. Au K, Lam D, Garg N, et al. Improving skills retention after advanced structured resuscitation training: A systematic review of randomized controlled trials. Resuscitation. 2019;138:284-296.

152. Bhatnagar V, Tandon U, Jinjil K, Dwivedi D, Kiran S, Verma R. Cardiopulmonary resuscitation: Evaluation of knowledge, efficacy, and retention in young doctors joining postgraduation program. Anesthesia, essays and researches. 2017;11(4):842.

153. Woollard M, Whitfield R, Newcombe RG, Colquhoun M, Vetter N, Chamberlain D. Optimal refresher training intervals for AED and CPR skills: A randomised controlled trial. Resuscitation. 2006;71(2):237-247.

154. Riegel B, Nafziger SD, McBurnie MA, et al. How Well are Cardiopulmonary Resuscitation and Automated External Defibrillator Skills Retained over Time? Results from the Public Access Defibrillation (PAD) Trial. Acad Emerg Med. 2006;13(3):254-263.

155. Saad R, Sampaio Favarato $M H$, Ferreira de Paiva E, do Patrocinio Tenorio Nunes M. Medical Student Skill Retention After Cardiopulmonary Resuscitation Training: A Cross-Sectional Simulation Study. Simul Healthc. 2019;14(6):351-358. 
156. Semeraro F, Signore L, Cerchiari EL. Retention of CPR performance in anaesthetists. Resuscitation. 2006;68(1):101-108. 


\section{Abbildungsverzeichnis}

Abbildung 1: Beispielhafte Darstellung der Rettungskette. Eigene Darstellung... 16 Abbildung 2: Todesursachen nach Krankheitsarten 2018, ............................. 17

Abbildung 3: Laienreanimation nach der GRC ……...................................... 19

Abbildung 4: Universitäre notfallmedizinische Ausbildung ............................... 26

Abbildung 5: Arbeitsschritte zur Curriculumsentwicklung ................................ 35

Abbildung 6: SimPad SkillReporter von Laerdal ......................................... 50

Abbildung 7: Schematische Darstellung des Studienprotokolls....................... 58

Abbildung 8: Grafische Darstellung der Schlüsselmaßnahmen der Reanimation 66

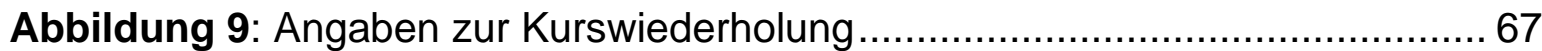

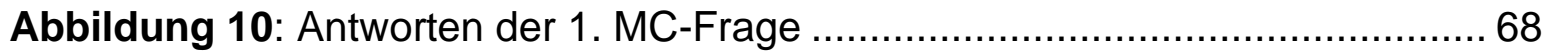

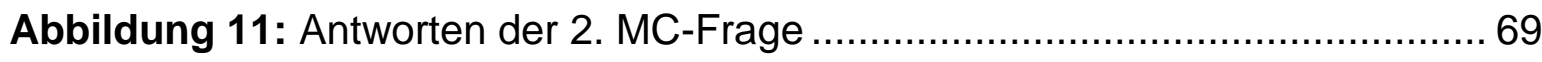

Abbildung 12: Antworten der 3. MC-Frage …………................................... 70

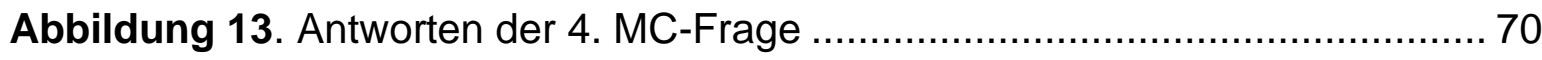

Abbildung 14: Antworten der 5. MC-Frage ……............................................ 71

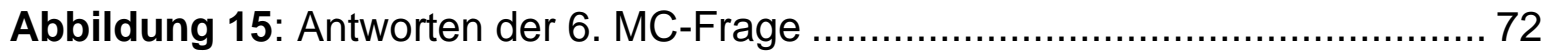

Abbildung 16: Antworten der 7. MC-Frage .................................................. 72

Abbildung 17: Antworten der 8. MC-Frage ..................................................... 73

Abbildung 18: Antworten der 9. MC-Frage .................................................... 74

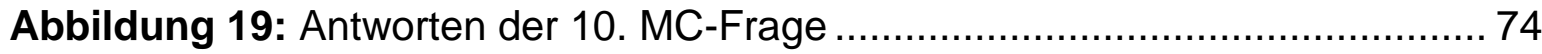

Abbildung 20: Schlüsselmaßnahmen der Reanimationen ............................... 77

Abbildung 21: Schlüsselmaßnahmen der Stabilen Seitenlage ......................... 79

Abbildung 22: Schlüsselmaßnahmen bei der Zwei-Helfer-Reanimation und Einsatz

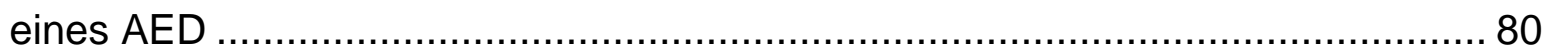

Abbildung 23: Grafische Darstellung der Kursevaluationen zu den allgemeinen Kursinhalten.

Abbildung 24: Grafische Darstellung der Kursevaluationen zu Struktur und Aufbau

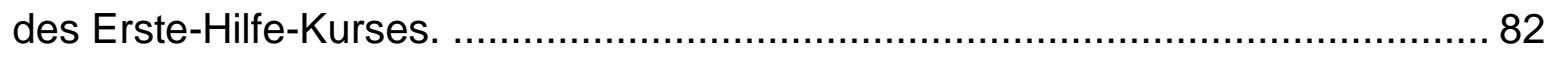
Abbildung 25: Grafische Darstellung der Kursevaluationen zu einzelnen Aspekten des Erste-Hilfe-Kurses. 83 
Abbildung 26: Grafische Darstellung der Kursevaluationen zu den Instruktor:innen. 84

Abbildung 27: Grafische Darstellung der Kursevaluationen zu Räumlichkeiten und

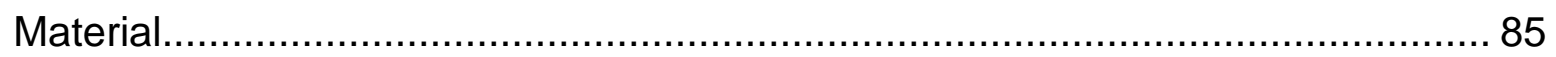

Abbildung 28: Abfrage zum Nutzen des ABCDE-Schemas im Notfall. ................. 86 Abbildung 29: Selbsteinschätzung der Erste-Hilfe-Kompetenz nach dem Erste-

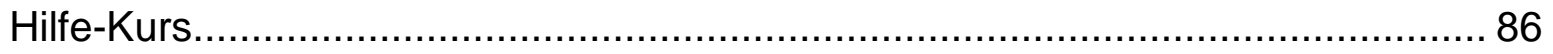




\section{Tabellenverzeichnis}

Tabelle 1: Erste-Hilfe-Kurse für Vorklinikstudierende an den medizinischen

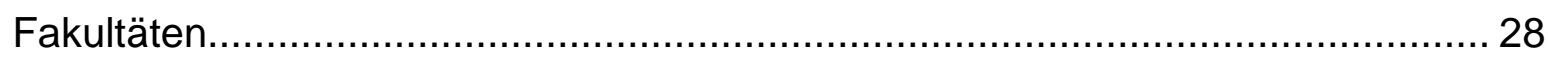

Tabelle 2: ABCDE-Schema ................................................................. 32

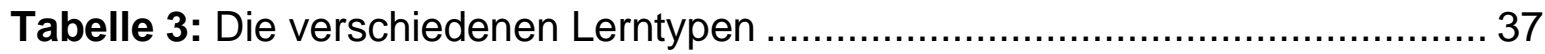

Tabelle 4: Notfallmedizinische Lernziele nach dem NKLM ................................. 39

Tabelle 5: Integrierte Kursinhalte des Erste-Hilfe-Kurses .................................. 40

Tabelle 6: Die 9 Unterrichtseinheiten des Erste-Hilfe-Kurses............................ 41

Tabelle 7: Fragen der Evaluation am Ende des Erste-Hilfe-Kurses.................... 46

Tabelle 8: Fragen des Selbsteinschätzungsbogens zum Messzeitpunkt 1 und 2. 47

Tabelle 9: Multiple-Choice-Fragen zum Messpunkt 1 und 2 ............................ 48

Tabelle 10: Die drei OSCE/OSPE-Stationen zum Messzeitpunkt 1 und 2........... 49

Tabelle 11: Erster Blueprint der drei OSCE-/OSPE-Stationen........................... 51

Tabelle 12: Checkliste der OSCE/OSPE-Station „Kardiopulmonale Ein-HelferReanimation“. 52

Tabelle 13: Ergänzung der Checkliste zur OSCE/OSPE-Station „Kardiopulmonale Ein-Helfer-Reanimation“........................................................................ 52

Tabelle 14: Checkliste der OSCE/OSPE-Station „Stabile Seitenlage“..................5 53

Tabelle 15: Checkliste der OSCE/OSPE-Station „Kardiopulmonale Zwei-Helfer-

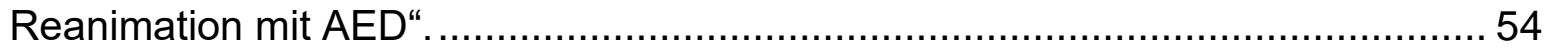

Tabelle 16: Einsatz von verschiedenen Simulatoren an den drei OSCE-/OSPE-

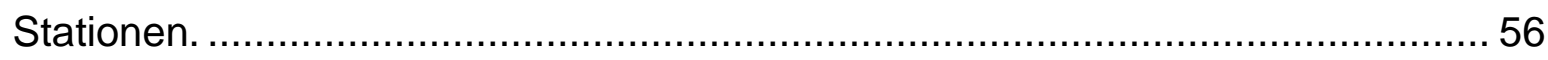

Tabelle 17: Anzahl der Studienteilnehmenden ............................................... 62

Tabelle 18: Demographische Daten der Teilnehmenden .................................. 64

Tabelle 19: Gründe der Teilnehmenden für die Teilnahme am Kurs .................... 64

Tabelle 20: Subjektive Einschätzung der Teilnehmenden ....................................6 65

Tabelle 21: Erreichte absolute Punktzahl an den 3 OSCE/OSPE-Stationen ........ 75

Tabelle 22: Kompetenzerfassung der OSCE/OSPE-Station „Ein-Helfer-

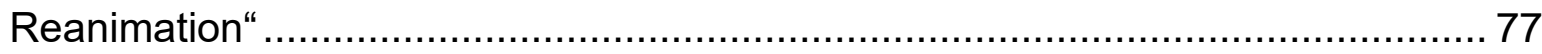

Tabelle 23: Kompetenzerfassung OSCE/OSPE-Station „Stabile Seitenlage“....... 78 
Tabelle 24: Kompetenzerfassung OSCE/OSPE-Station „Zwei-Helder-Reanimation, Einsetzen eines AED und Anleitung eines Passanten“ 80 Tabelle 25: Tabellarische Darstellung der Evaluation zu den allgemeinen

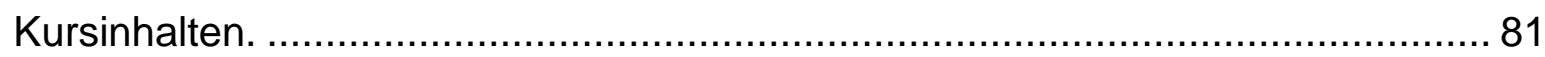
Tabelle 26: Tabellarische Darstellung der Kursevaluationen zu Struktur und Aufbau

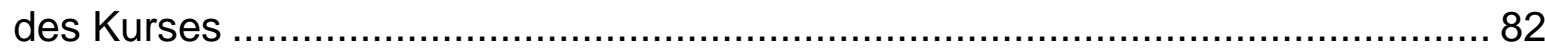
Tabelle 27: Tabellarische Darstellung der Kursevaluationen zu einzelnen Aspekten

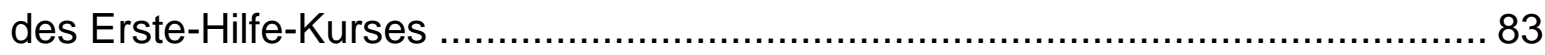
Tabelle 28: Tabellarische Darstellung der Kursevaluationen zu den Instruktor:innen 84

Tabelle 29: Tabellarische Darstellung der Kursevaluationen zu Räumlichkeiten und Material 85 


\section{Anhang}

\subsection{Kursunterlagen}

\subsubsection{Kursablaufplan Stand 2017}

\section{Tagesablauf}

\begin{tabular}{|c|c|c|}
\hline UE & Zeit & \\
\hline $1 \mathrm{UE}$ & 9:00-9:45 Uhr & $\begin{array}{l}\text { - Begrüßung: Dozenten-, TN-Vorstellung, warum brauchen wir Erste-Hilfe etc. } \\
\text { - Rechtliche Einweisung } \\
\text { - Rettungskette erklären } \\
\text { Erstes Glied der Rettungskette } \rightarrow \text { Scene - Safety - Situation } \\
\text { - Verhalten an der Einsatzstelle: } \\
\circ \quad \text { Absichern, eigene Sicherheit/eigenes Schutzverhalten } \\
\circ \quad \text { Rettung aus einem Gefahrenbereich } \\
\text { - General Impression } \\
\circ \quad \text { Vital gefährdet/nicht vital gefährdet } \\
\circ \text { Notruf absetzen }\end{array}$ \\
\hline $2 \mathrm{UE}$ & $9: 55-10: 40 \mathrm{Uhr}$ & $\begin{array}{l}\text { Zweites Glied der Rettungskette } \rightarrow \text { Erste Hilfe } \\
\text { Airway } \\
\text { - } 10 \text { Sekunden Überprüfung der Atmung } \\
\text { Breathing } \\
\text { - AF abschätzen, Atemgeräusche } \\
\text { - Pathologische Atmungsformen, respiratorische Störungen, Krankheitsbilder } \\
\text { - Keine Atmung und kein Puls } \rightarrow \text { Reanimation, Übergang zur HLW }\end{array}$ \\
\hline $3 \mathrm{UE}$ & $10: 50-11: 35 \mathrm{Uhr}$ & $\begin{array}{l}\text { Herz-Lungen-Wiederbelebung unter Einsatz eines AED } \\
\text { - Schockbare vs. Nicht-schockbare Rhythmen } \\
\text { - Beatmung }\end{array}$ \\
\hline $4 \mathrm{UE}$ & $11: 45-12: 30 \mathrm{Uhr}$ & Herz-Lungen-Wiederbelebung unter Einsatz eines AED \\
\hline & $12: 30-13: 15 \mathrm{Uhr}$ & Mittagpause \\
\hline $5 \mathrm{UE}$ & 13:15-14:00Uhr & $\begin{array}{l}\text { - Kontrolle des Bewusstseins durchführen und Gefahren der Bewusstlosigkeit kennen } \\
\text { - Motoradfahrer: Helmabnahme beim bewusstlosen Motorradfahrer } \rightarrow \text { TÜ } \\
\text { - Seitenlage durchführen } \rightarrow \text { TÜ }\end{array}$ \\
\hline $6 \mathrm{UE}$ & $14: 10-14: 55$ Uhr & $\begin{array}{l}\text { - Circulation } \\
\circ \text { Schock } \\
\circ \text { Exkurs: Koronare Herzkrankheiten, EKG kleben }\end{array}$ \\
\hline $7 \mathrm{UE}$ & $15: 05-15: 50 \mathrm{Uhr}$ & $\begin{array}{l}\text { - Disability } \\
\circ \text { hirnbedingte Störungen erkennen, entsprechende Maßnahmen bei Schlaganfall } \\
\text { und Krampfanfall } \\
\circ \text { Hypoglykämie } \\
\text { - Exposure, Environment } \\
\circ \quad \text { Lagerungsarten: atemerleichternde Lagerung, Oberkörperhochlagerung } \\
\circ \text { Maßnahmen zum Wärmeerhalt } \\
\circ \text { Suche nach weiteren Verletzungen } \rightarrow \text { Übergang zu Secondary Survey }\end{array}$ \\
\hline & $15: 50-16: 10 \mathrm{Uhr}$ & Kaffeepause \\
\hline $8 \mathrm{UE}$ & $16: 10-16: 55$ Uhr & $\begin{array}{l}\text { Drittes Glied der Rettungskette: Secondary Survey } \\
\text { - Erheben von Vitalparametern, Bodycheck etc. } \\
\text { - Wundversorgung mit Verbandmitteln aus dem Verbandkasten } \\
\circ \text { Bedrohliche, spritzende Blutungen erkennen } \rightarrow \text { Druckverband } \\
\circ \quad \text { Versorgung von Amputationsverletzungen und weitere Besonderheiten: z.B. } \\
\text { Fremdkörper in Wunden, Nasenbluten, Verbrennungen }\end{array}$ \\
\hline 9 UE & $17: 05-17: 50 \mathrm{Uhr}$ & $\begin{array}{l}\text { - Maßnahmen bei Knochenbrüchen und Gelenkverletzungen } \\
\text { - Maßnahmen zur psychischen Betreuung } \\
\text { Viertes und fünftes Glied der Rettungskette } \rightarrow \text { Rettungsdienst und Krankenhaus } \\
\text { - Zusammenarbeit } \\
\text { - Ausblick }\end{array}$ \\
\hline
\end{tabular}




\subsubsection{Ausbildungs-Nachweis Instruktoren Stand 2017}

Erläuterungen zum Ausbildungsnachweis für Erste-Hilfe-Instruktoren

Du bist als studentischer Instruktor für das FinesT tátie

Damit du erfolgreich und mit einem gewissen MaB an Erfahrung diese verantwortungsvolle Tătigkeit bewăltigen kannst, musst du das standardisierte Ausbildungs-Curriculum durchlaufen.

Hierzu gehören sowohl Hospitationen in unseren Kursen, als auch Einweisungen an Medizinischen Produkten sowie Praxistermine im aktiven Rettungsdienst.

- Solltest du eine der oben genannten Ausbildungen haben, reicht eine Vorlage des Ausbildungs-Zeugnisses. Auf Wunsch kannst du natürlich zusătzlich die oben genannten Praktika (RTW, NEF) wahrnehmen.

Mit den Kenntnissen aus deiner Ausbildung kannst du dichim Ratmen deiner Aufgoben schnell produktiv in die Kurse einbringen. In diesem Ausbildungsnachweis, sind eine Reihe dieser Tätigkeiten aufgeführt, die du dir von den Kursleitern oder verantwortlichen Mitarbeitern mittels Handzeichen bescheinigen lässt.

Wir möchten dich auch gerne zu den Gruppensitzungen des Finest sowie zu den Treffen des Erste-Hilfe-Ausbilderkreises einladen und würden uns über eine regelmáßige Teilnahme freuen. Dies dient dem Austausch untereinander, def ständigen Evaluation unserer Kurse und des Konzeptes sowie der Verbesserune unserer lehre.

Benötigte Arbeits-, Finsatzkleidung und Selbstschutrausrustung wird dir vom FineSI zur Verfügung gestellt.

Generell gilt, dass wăhrend der Kurse die Weisungsbefugnis und damit auch die Verantwortung für Maßnahmen bei den Instruktoren des Kurses liegen. Bitte achtet darauf, dass:

1. unsere Studierenden keinen Schaden nehmen

unser Material nicht unnotig beschadigt oder verschwendet wird

du und deine Kollegen ein sauberes und sicheres Arbeitsumfeld haben

. Keine unbefugten Personen Zutritt zu unseren Räumen erhalten (Hausrecht)

Bitte wendet euch bei Fragen, konstruktiver Kritik, Schwierigkeiten oder Anmerkungen

Viel Erfolg und viel Spaß an der Arbeit im Finest wūnsch

Das Team des FlnesT \& der Arbeitskreis des Erste-Hilfe-Kurses

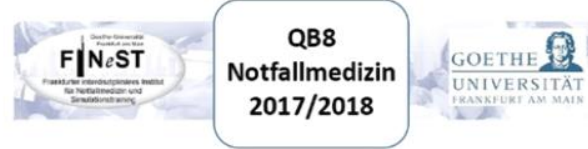

Ausbildungsnachweis Erste-Hilfe-Instruktor

am FIneST

Name
Basic Life Support - Tag 1

am

O Vor- und Nachbereitung selbststāndig erledigt

Basic Life Support - Tag 2

Verantwortlicher instruktor

O Vor- und Nachbereitung selbststăndig erledigt

Train The Trainer (Methodik-Didaktik)

am Verantwortlicher Instruktor

Hospitationen im Erste-Hilfe-Kurs

$1 \times$ Teilnahme als Hospitant

am Teinahme ais Hospitant

oVor- und Nachbereitung selbstständig erledigt

$1 \times$ Teilnahme als Tutor

Unter Aufsicht von

RTW-Praktikum (2 Tage)

Freiwilliges NEF-Praktikum

Zeitraum

Oder: Ausbildungsbescheinigung liegt in Kopie vor (s. Erläuterungen) O Notfallsanită̌ter O Rettungsassistent ORettungssanitäter

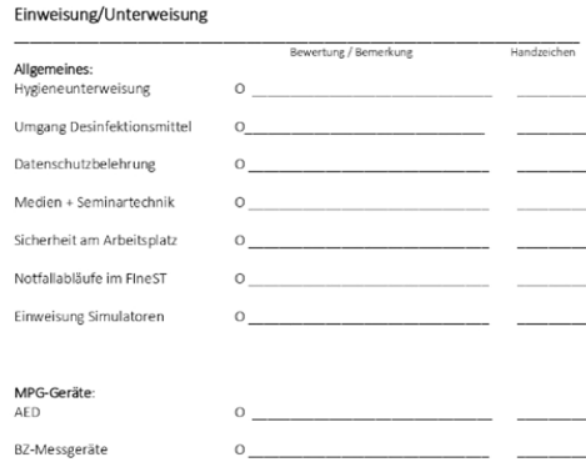

Sonstige relevante Bemerkungen und/oder Maßnahmen:

Freigabe

Datum Name und Handzeichen 


\subsubsection{Teilnahmebescheinigung Stand 2017}

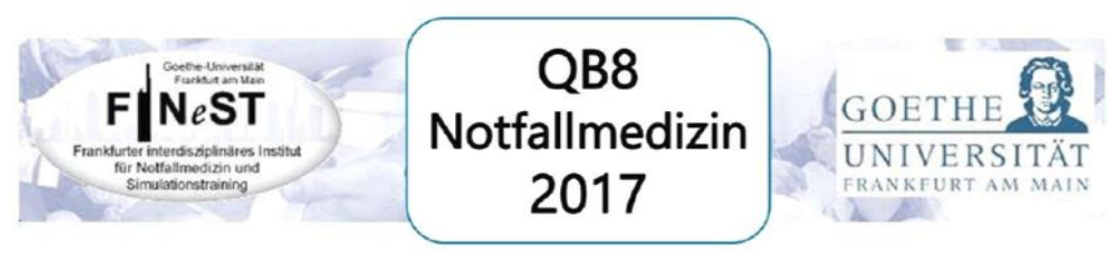

\section{Teilnahmebescheinigung Erste Hilfe Kurs}

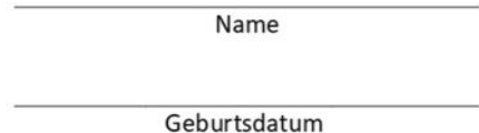

Hat am an einem 9 UE umfassenden Erste-Hilfe-Kurs teilgenommen.

Zu den Ausbildungsinhalten gehörte unter anderem:

- Struktur der Rettungskette

- Eigene Sicherheit, Rettung aus einem Gefahrenbereich

- Notruf absetzen

- Gefahren bei Atemstillstand und entsprechende Maßnahmen

- Herz-Lungen-Wiederbelebung unter Einsatz eines AED

- Kontrolle des Bewusstseins, Gefahren der Bewusstlosigkeit kennen, Seitenlage durchführen

- Helmabnahme beim bewusstlosen Motorradfahrer

- kreislaufbedingte Störungen erkennen, entsprechende Maßnahmen bei Herzinfarkt

- hirnbedingte Störungen erkennen, entsprechende Maßnahmen bei Schlag- und Krampfanfall

- Wundversorgung mit Verbandmitteln, Druckverband, Versorgung Amputationsverletzungen

Frankfurt, den 


\title{
9.2 Prüfermappe
}

\subsubsection{Infotext Prüfer}

\section{Infotext Prüfer}

\begin{abstract}
Prüfer
Die Aufgabe der Prüfer ist es, die Studierenden anhand eines Prüfungsbogens in den 3 kleinen praktischen Stationen zu bewerten. Diese kurze Checkliste dient zur Überprüfung verschiedener Items, die die Studierenden in dem jeweiligen Szenario erbringen sollten. In der Checkliste gibt es eine Punkteskala, sollte die Maßnahme erfolgt sein bitte ankreuzen. Bei 2 Punkten muss entschieden werden, ob die Maßnahme komplett (2 Punkte) oder teilweise (1 Punkt) durchgeführt wurde.

Sollten weitere Anmerkungen von Prüferseite aus nötig sein, bitte auf dem Prüfungsbogen des Studierenden vermerken.
\end{abstract}

\section{Assistenten}

Die Aufgabe des Assistenten ist es, den Studierenden den Weg durch den Mini-OSCE zu erleichtern. Sie nehmen die Zeit und pfeifen, sobald es Zeit ist eine Station zu wechseln. An den Stationen selbst weisen sie die Richtung, beantworten allerdings keine inhaltlichen Fragen. 


\subsubsection{OSCE-Checklisten}

\section{Prüfungsbogen Situation 1}

Teilnehmer-Nummer Name

Prüfer-Name Uhrzeit:

\begin{tabular}{|l|l|l|}
\hline Ausschließen Eigengefährdung & 1 & \\
\hline Ansprechen & 1 & \\
\hline vorsichtiges Schütteln an der Schulter & 1 & \\
\hline „um Hilfe rufen“ & 1 & \\
\hline Atemwege freimachen & & \\
- Kopf reklinieren & 1 & \\
- Kinn vorziehen & 1 & \\
\hline Atemkontrolle & 1 & \\
- Sehen, Hören, Fühlen & 1 & \\
- Zeitdauer max. 10 Sekunden & 2 & \\
\hline Veranlassen eines Notrufs & 1 & \\
\hline Aufsuchen des korrekten Druckpunktes & 1 & \\
\hline korrekte Hand- und Körperhaltung & 1 & \\
\hline 30 Herzdruckmassagen & 2 & \\
- Frequenz: 100-120 Kompressionen/min & 2 & \\
- Drucktiefe: 5-6 cm & 1 & \\
- Kompression \& Entlastung gleich lang, vollständige Entlastung & & \\
\hline
\end{tabular}

Sobald der TN mit den Herzdruckmassagen fertig ist und genügend Zeit bleibt:

\section{Durchdrücken lassen}

$\begin{array}{lllll}\text { Frequenz } & 80 \% & 2 \text { Punkte } & 60 \% & 1 \text { Punkt } \\ \text { Drucktiefe } & 80 \% & 2 \text { Punkte } & 60 \% & 1 \text { Punkt } \\ \text { Kompression \& Entlastung } & >80 \% & 1 \text { Punkt } & & \end{array}$




\section{HINWEIS:}

Falls der TN genügend Zeit hat, lasst ihr ihn durchdrücken und beurteilt am SimPad die Leistung.

Ihr vergebt die Punkte folgendermaßen:

Frequenz

Drucktiefe

Kompression \& Entlastung
$80 \% \quad 2$ Punkte

$60 \% 1$ Punkt

$80 \% \quad 2$ Punkte

$60 \% 1$ Punkt

$80 \% 1$ Punkt 


\section{Prüfungsbogen Situation 2}

$\begin{array}{lll}\text { Teilnehmer-Nummer__ Name__ } & \\ \text { Prüfer-Name __ Uhrzeit: } & \end{array}$

\begin{tabular}{|l|l|l|}
\hline Ausschließen Eigengefährdung & 1 & \\
\hline Ansprechen & 1 & \\
\hline vorsichtiges Schütteln an der Schulter & 1 & \\
\hline „um Hilfe rufen“ & 1 & \\
\hline $\begin{array}{l}\text { Atemwege freimachen } \\
\text { - Kopf reklinieren }\end{array}$ & & \\
- Kinn vorziehen & 1 & \\
\hline $\begin{array}{l}\text { Atemkontrolle } \\
\text { - Sehen, Hören, Fühlen } \\
\text { - Zeitdauer max. 10 Sekunden }\end{array}$ & 1 \\
\hline Veranlassen eines Notrufs & 1 \\
\hline $\begin{array}{l}\text { Lagerung der Patientin in Rückenlage, } \\
\text { Position des Studierenden kniend seitlich der Patientin }\end{array}$ & 1 & \\
\hline Korrekte Lagerung in eine stabile Seitenlage ohne die Patientin zu verletzen & 1 & \\
\hline Überstrecken des Kopfes und Öffnen des Mundes & 1 & \\
\hline
\end{tabular}




\section{Prüfungsbogen Situation 3}

Teilnehmer-Nummer

Name

Prüfer-Name

Uhrzeit:

\begin{tabular}{|l|l|l|}
\hline Ausschließen Eigengefährdung & 1 & \\
\hline Startet mit der CPR & 1 & \\
\hline Bindet den Passanten sinnvoll bei der CPR ein & 1 & \\
\hline Setzt den AED ein & 2 & \\
\hline korrekte Befolgung der Anweisungen & 1 & \\
\hline $\begin{array}{l}\text { Beachtet eine sinnvolle Reihenfolge (1;2;3;4): } \\
\text { Anschalten ( ), Aufkleben ( ), Anschließen ( ), Analyse ( ) }\end{array}$ & 2 & \\
\hline Gibt Kommando: Alle weg vom Patienten & 1 & \\
\hline Kontrolle, dass keiner den Patient berührt & 2 & \\
\hline Gibt Kommando: Achtung Schock & 1 & \\
\hline Auslösung des Schocks & 1 & \\
\hline $\begin{array}{l}\text { Startet die weitere zügig weiter mit CPR } \\
\text { verzögert (_min) }\end{array}$ & 1 & \\
\hline
\end{tabular}




\title{
9.3 Studierendenmappe
}

\subsubsection{Subjektive Einschätzung vor und nach dem OSCE}

\section{Subjektive Einschätzung vor und nach dem OSCE}

1. Wenn jemand einen Herzstillstand in deiner Gegenwart erleidet, würdest du Wiederbelebungsmaßnahmen durchführen?

$\square \mathrm{Ja}$

$\square$ Nein

\author{
2. Falls nein, würdest du nicht wiederbeleben, weil: (Mehrfachantworten möglich) \\ 口Ich Mund zu Mund beatmen müsste \\ $\square$ Ich Angst vor einer Infektion habe \\ $\square$ Ich Angst habe, den Menschen zu verletzen \\ $\square$ Ich Angst habe, hinterher rechtlich belangt zu werden \\ 口ich nicht weiß, wie ich wiederbeleben kann
}
3. Fühlst du dich momentan in der Lage Wiederbelebungsmaßnahmen durchzuführen?
$\square \mathrm{Ja}$
$\square$ Nein

4. Wie sicher fühlst du dich in den einzelnen Maßnahmen?

$\begin{array}{llll}\text { Ansprechen: } & \square \text { sehr sicher } & \square \text { sicher } & \square \text { unsicher } \\ \text { Hilfe rufen: } & \square \text { sehr sicher } & \square \text { sicher } & \square \text { unsicher } \\ \text { Atmung überprüfen: } & \square \text { sehr sicher } & \square \text { sicher } & \square \text { unsicher } \\ \text { Notruf absetzen: } & \square \text { sehr sicher } & \square \text { sicher } & \square \text { unsicher } \\ \text { Herzdruckmassage: } & \square \text { sehr sicher } & \square \text { sicher } & \square \text { unsicher } \\ \text { Anwendung eines AED: } & \square \text { sehr sicher } & \square \text { sicher } & \square \text { unsicher } \\ \text { Beatmung: } & \square \text { sehr sicher } & \square \text { sicher } & \square \text { unsicher }\end{array}$

5. Wie oft sollte ein solcher Kurs deiner Meinung nach wiederholt werden?

口alle 6 Monate $\square$ einmal im Jahr $\square$ alle 2 Jahre

$\square$ alle 5 Jahre $\quad \square$ alle 10 Jahre $\square$ gar nicht

6. Erachtest du es als sinnvoll, einen Erste-Hilfe-Kurs als Zulassungsvoraussetzung für das Physikum absolvieren zu müssen?

$\square$ Ja $\quad \square$ Nein 


\subsubsection{MC-Fragen vor und nach dem OSCE}

\section{MC-Fragen vor und nach dem OSCE}

1. Du siehst jemanden kollabieren, wie gehst du als erstes vor?

$\square$ Den Mund des Patienten überprüfen

$\square$ Prüfen, ob der Patient auf Ansprache reagiert

$\square 2 \mathrm{x}$ beatmen

$\square$ Das Heimlich-Manöver ausführen

2. Wie lange versuchst du herauszufinden, ob die Person einen Kreislauf hat?

$\square 5$ Sekunden

$\square 10$ Sekunden

$\square 15$ Sekunden

$\square 20$ Sekunden

3. Wie schnell drückt man bei der Herzdruckmassage?

$\square$ 60-80 mal/Minute

$\square$ 80-100 mal/Minute

$\square$ 100-120 mal/Minute

$\square 120-140 \mathrm{mal} /$ Minute

4. Wie viele Herzdruckmassagen führst du im Rahmen eines Zyklus beim Herzkreislaufstillstand durch?

$\square 5$

$\square 15$

$\square 30$

$\square 40$

5. Welches der folgenden Zeichen ist KEIN sicheres Zeichen für eine Fraktur?

$\square$ sichtbare Knochenenden

$\square$ Krepitation

$\square$ Bewegungseinschränkung

$\square$ abnorme Beweglichkeit

6. Anzeichen Schlaganfall

$\square$ Bewusstseinsstörung

$\square$ Sprachstörung

$\square$ Halbseitenlähmung/-schwäche

$\square$ Sehstörung alle Antworten sind korrekt 
7. Im Supermarkt triffst du auf einen Kunden, der über starke Brustschmerzen klagt. Wie gehst du weiter mit inm vor?

$\square$ Ich lege die Person in die stabile Seitenlage.

$\checkmark$ Ich lagere die Person so, dass der Oberkörper möglichst erhöht ist.

$\square$ Die Person soll sich flach hinlegen.

$\square$ Ich muss nichts tun.

$\square$ Die Person soll den Hausarzt aufsuchen.

8. Du bist Zeuge eines Verkehrsunfalls. Ein Motorradfahrer liegt bewusstlos auf der Straße. Du nimmst den Helm...

$\square$... ab um die Atmung adäquat kontrollieren zu können.

$\square$... nicht ab um Manipulationen und Verletzungen zu vermeiden.

$\square$... ab um eine stabile Seitenlage durchführen zu können.

9. Du versorgst eine stark blutende Wunde, wie gehst du dabei als erstes vor?

$\square$ Ich versuche die Blutung durch Abdrücken mit der Hand zu stoppen.

$\checkmark$ Ich versuche durch Abbinden die Wunde zu versorgen.

$\checkmark$ Ich lege einen Druckverband an und versuche so, die Blutung zu stoppen.

$\square$ Keine der Aussagen trifft zu.

10. Du sollst einen Notruf absetzen, welche Nummer wählst du?

$\square 112$

$\square 110$

口 911

口"069" 


\subsubsection{Informationstext der 3 OSCE-Stationen}

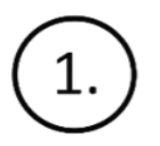

Situation: Du hilfst gerade im Pflegepraktikum einem Patienten beim ankleiden, als sein Zimmernachbar beim Versuch aufzustehen plötzlich zu Boden stürzt. Er bleibt reglos auf dem Boden liegen. Die Ursache für den Sturz ist nicht erkennbar.

Aufgabe: Gehe zu dem Patienten und stelle fest, in welchem Zustand er sich befindet. Führe dann erforderliche Maßnahmen durch. 


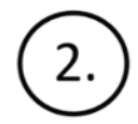

Situation: Im Biochemie-Praktikum wirst du Zeuge eines Kollapses einer Kommilitonin. Sie bleibt regungslos auf dem Boden liegen.

Aufgabe: Kontrolliere bei ihr die Atmung und führe die erforderlichen Erstmaßnahmen durch.

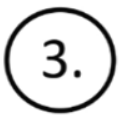

Situation: Du findest eine leblose Person vor, sie atmet nicht mehr. Der Notruf wurde bereits abgesetzt und ein weiterer Passant ist anwesend.

Aufgabe: Beginne mit der Reanimation und setze Hilfsmittel, die du zusätzlich für die Wiederbelebung nutzen kannst, ein. 


\subsubsection{Abschlussevaluationsbogen}

\section{Abschlussevaluationsbogen}

Allgemeines zum Kurs

1. Wie bewertet Ihr den Kurs insgesamt?

$\square$ sehrgut $\quad \square$ gut $\quad \square$ mittelmäßig $\square$ schlecht $\square$ sehr schlecht

2. Wie war die Atmosphäre im Kurs?

$\square$ sehrgut $\quad \square$ gut $\square$ mittelmäßig $\square$ schlecht $\square$ sehr schlecht

3. Wie war das Auftreten/die Kommunikation des Instruktors?

$\square$ sehrgut $\quad \square$ gut $\square$ mittelmäßig $\square$ schlecht $\square$ sehr schlecht

4. Wie habt ihr Euch in unseren Räumlichkeiten gefühlt?

$\square$ sehr gut $\quad \square$ gut $\quad \square$ mittelmäßig $\square$ schlecht $\square$ sehr schlecht

Struktur und Aufbau des Kurses

1. Die Dauer des Kurses war insgesamt

$\square$ zu lang $\square$ länger als geplant $\square$ optimal $\square$ kürzer als geplant $\square$ zu kurz

2. Der Praxisanteil war

$\square$ zu lang $\square$ länger als geplant $\square$ optimal $\square$ kürzer als geplant $\square$ zu kurz

3. Der Theorieanteil war

$\square$ zu lang $\quad \square$ länger als geplant $\square$ optimal $\quad \square$ kürzer als geplant $\quad \square$ zu kurz

4. Die Pausen waren

$\square$ zu lang $\quad \square$ länger als geplant $\square$ optimal $\quad \square$ kürzer als geplant $\square$ zu kurz

Das Erste-Hilfe Training war

1. Theoretisch interessant

$\square$ Trifft zu $\quad \square$ Trifft eher zu $\quad \square$ Teils-Teils $\quad \square$ Trifft eher nicht zu $\quad \square$ Trifft nicht zu

2. Notwendig

$\square$ Trifft zu $\quad \square$ Trifft eher zu $\quad \square$ Teils-Teils $\quad \square$ Trifft eher nicht zu $\quad \square$ Trifft nicht zu

3. Praktisch für mich persönlich umsetzbar

$\square$ Trifft zu $\quad \square$ Trifft eher zu $\quad \square$ Teils-Teils $\quad \square$ Trifft eher nicht zu $\quad \square$ Trifft nicht zu

4. Meinen Vorstellungen entsprechend

$\square$ Trifft zu $\quad \square$ Trifft eher zu $\quad \square$ Teils-Teils $\quad \square$ Trifft eher nicht zu $\quad \square$ Trifft nicht zu 
Der/Die Instruktor*in

1. Hat klar und deutlich gesprochen

$\square$ Trifft zu $\quad \square$ Trifft eher zu $\quad \square$ Teils-Teils $\quad \square$ Trifft eher nicht zu $\quad \square$ Trifft nicht zu

2. Geht angemessen auf Anregungen und Fragen ein

$\square$ Trifft zu $\quad \square$ Trifft eher zu $\quad \square$ Teils-Teils $\quad \square$ Trifft eher nicht zu $\quad \square$ Trifft nicht zu

3. Erklärt Sachverhalte und Zusammenhänge verständlich

$\square$ Trifft zu $\quad \square$ Trifft eher zu $\quad \square$ Teils-Teils $\quad \square$ Trifft eher nicht zu $\quad \square$ Trifft nicht zu

4. Vermittelt den Stoff in einem angenehmen Tempo

$\square$ Trifft zu $\quad \square$ Trifft eher zu $\quad \square$ Teils-Teils $\quad \square$ Trifft eher nicht zu $\quad \square$ Trifft nicht zu

Räumlichkeiten und Material

1. Der Kursraum war groß genug

$\square$ Trifft zu $\quad \square$ Trifft eher zu $\quad \square$ Teils-Teils $\quad \square$ Trifft eher nicht zu $\quad \square$ Trifft nicht zu

2. Die Anzahl der Teilnehmer*innen war angemessen

$\square$ Trifft zu $\quad \square$ Trifft eher zu $\quad \square$ Teils-Teils $\quad \square$ Trifft eher nicht zu $\quad \square$ Trifft nicht zu

3. Es gab ausreichend Material zum Üben

$\square$ Trifft zu $\quad \square$ Trifft eher zu $\quad \square$ Teils-Teils $\quad \square$ Trifft eher nicht zu $\quad \square$ Trifft nicht zu

Ich fühle mich nach dem Kurs in der Lage, professionell Erste-Hilfe zu leisten

$\square$ Trifft zu $\quad \square$ Trifft eher zu $\quad \square$ Teils-Teils $\quad \square$ Trifft eher nicht zu $\quad \square$ Trifft nicht zu

Würdest Du den Kurs weiterempfehlen und wenn ja warum, bzw. warum nicht?

Hilft dir das vorgestellte ABCDE-Schema im Notfall?

$\square \mathrm{Ja} \quad \square$ Nein 


\section{Danksagung}

Ich danke Frau Prof. Dr. Rüsseler für ihre herausragende Betreuung, Unterstützung und ständige Erreichbarkeit bei Fragen aller Art. Sie hat mich immer wieder motiviert und ermöglicht, dass das Projekt des Erste-Hilfe-Kurses für Vorklinikstudierende umgesetzt wurde. Sie ist eine großartige Mentorin, absolutes Vorbild und ich bin froh, dass ich ihre Doktorandin sein durfte.

Des Weiteren danke ich Kristina Gerbracht, Barbara Durekovic und Jan Heusel, die durch den Besuch ihres Erste-Hilfe-Kurses vor dem Physikum 2016 den Anstoß zu dieser Arbeit geliefert haben. Wären eure Beschwerden über diesen Tag nicht so groß gewesen, hätte ich das Projekt vielleicht nie gestartet.

Ein Dank gehört auch den teilnehmenden Studierenden dieser Studie, die so bereitwillig teilgenommen haben und ohne die diese Studie nicht möglich gewesen wäre.

Ein besonderer Dank gilt dem FIneST-Team, die durch ihre Arbeit und Motivation den Erste-Hilfe-Kurs in dieser Form möglich gemacht haben und immer noch möglich machen: Barbara Durekovic, Svea Linßen, Jan Kasperek, Michael Zentgraf, Lukas Thorsson, Simon Kusch, Madara Möhlmann, Lukas Müllerleile, Finn Lunscken und viele, viele andere. Allen Beteiligten danke ich von ganzem Herzen für eure Arbeit und das Vertrauen, ihr seid die Besten!

Herrn Bernd Bender danke ich für seine unendliche Geduld, mit der er mich in die Tiefen der Statistik eingeführt hat.

Ich danke meiner Familie für einfach alles, insbesondere den ständigen Glauben an mich.

Und schlussendlich möchte ich meiner einzigartigen WG mit den besten Freundinnen danken. Fürs zuhören, Kritik üben, ausbremsen, begeistert durchlesen und vor allem für die vielen gemeinsamen Kaffeetassen.

Ohne euch alle wäre diese Dissertation nicht möglich gewesen. 
11 Curriculum Vitae 


\section{Veröffentlichung und Vorträge}

\subsection{Publikationen}

Lorenz J.; Durekovic B.; Kasperek J.; Bender, B..; Rüsseler M.: „Einfluss eines Erste-Hilfe-Kurses für Medizinstudierende auf notfallmedizinische Basiskompetenzen“ German Medical Science GMS Publishing House; DocV11-02. DOI: 10.3205/19gma082. Düsseldorf, 20. September 2019

\subsection{Vorträge}

Lorenz J.; Durekovic B.; Kasperek J.; Bender, B..; Rüsseler M.: „Einfluss eines ErsteHilfe-Kurses für Medizinstudierende auf notfallmedizinische Basiskompetenzen" Gemeinsame Jahrestagung der Gesellschaft für Medizinische Ausbildung (GMA), des Arbeitskreises zur Weiterentwicklung der Lehre in der Zahnmedizin (AKWLZ) und der Chirurgischen Arbeitsgemeinschaft Lehre (CAL). Frankfurt am Main, 25.28.09.2019

\subsection{Poster}

Lorenz J.; Durekovic B.; Kasperek J.; Bender, B..; Rüsseler M.: „Einfluss eines ErsteHilfe-Kurses für Vorklinik-Studierende auf notfallmedizinische Basiskompetenzen“ 10. Deutscher Interdisziplinärer Notfallmedizin Kongress. Koblenz, 21. - 22. März 2019

Lorenz J.; Durekovic B.; Kasperek J.; Zentgraf M.; Sterz, J.; Rüsseler M.: „Statuserhebung notfallmedizinischer Basiskompetenzen bei Studierenden der Humanmedizin im vorklinischen Studienabschnitt“ 17. Jahreskongress der Deutschen Interdisziplinären Vereinigung für Intensiv- und Notfallmedizin. Leipzig, 06.- 08.12.2017

Lorenz J.; Durekovic B.; Kasperek J.; Zentgraf M.; Sterz, J.; Rüsseler M.: „Einfluss eines Erste-Hilfe-Kurses in der Vorklinik auf notfallmedizinische Basiskompetenzen“" 17. Jahreskongress der Deutschen Interdisziplinären Vereinigung für Intensiv- und Notfallmedizin. Leipzig, 06.- 08.12.2017 


\section{Schriftliche Erklärung}

Ich erkläre ehrenwörtlich, dass ich die dem Fachbereich Medizin der Johann Wolfgang Goethe-Universität Frankfurt am Main zur Promotionsprüfung eingereichte Dissertation mit dem Titel „Einfluss eines Erste-Hilfe-Kurses für Medizinstudierende auf notfallmedizinische Basiskompetenzen" am Zentrum für Chirurgie, Klinik für Unfall-, Hand- und Wiederherstellungschirurgie unter Betreuung und Anleitung von Prof. Dr. Miriam Rüsseler ohne sonstige Hilfe selbst durchgeführt und bei der Abfassung der Arbeit keine anderen als die in der Dissertation angeführten Hilfsmittel benutzt habe. Darüber hinaus versichere ich, nicht die Hilfe einer kommerziellen Promotionsvermittlung in Anspruch genommen zu haben. Ich habe bisher an keiner inoder ausländischen Universität ein Gesuch um Zulassung zur Promotion eingereicht. Die vorliegende Arbeit wurde bisher nicht als Dissertation eingereicht.

Vorliegende Ergebnisse der Arbeit wurden (oder werden) in folgendem Publikationsorgan veröffentlicht:

Lorenz J.; Durekovic B.; Kasperek J.; Bender, B..; Rüsseler M.: „Einfluss eines ErsteHilfe-Kurses für Medizinstudierende auf notfallmedizinische Basiskompetenzen" German Medical Science GMS Publishing House; DocV11-02. DOI: 10.3205/19gma082. Düsseldorf, 20. September 2019

Ort, Datum

Unterschrift 